\title{
Nitrogen-containing five-membered heterocyclic compounds as corrosion inhibitors for metals in solutions of mineral acids - An overview
}

\author{
Ya.G. Avdeev and Yu.I. Kuznetsovi(i) \\ A.N. Frumkin Institute of Physical Chemistry and Electrochemistry, Russian Academy \\ of Sciences, Leninskii pr. 31, 119071 Moscow, Russian Federation \\ E-mail: avdeevavdeev@mail.ru
}

\begin{abstract}
The review describes the current state of studies in the field of protection of ferrous and nonferrous metals from corrosion in solutions of mineral and organic acids by $\mathrm{N}$-containing five-membered heterocyclic compounds (NFHC). Literature data on the protection of various metals (steels, copper-, zinc-, and aluminum-based alloys) by these corrosion inhibitors (CIs) in acid solutions are generalized. The specifics of the mechanism of their protective effect are discussed. Analysis of data on the free adsorption energy of NFHC on the surface of various metals in acid media often allows assuming the chemisorption character of their interaction, which to a great extent explains the efficiency of these compounds in corrosion inhibition. The unique ability of composite NFHC-based CIs to protect metals in extremely corrosive media (high temperature acid solutions ( $t$ up to $200^{\circ} \mathrm{C}$ ) and acid solutions containing $\mathrm{Fe}$ (III) salts) is discussed. The ability of such composite CIs to inhibit corrosion in such corrosive media is based on the ability of NFHCs to form polymolecular protective layers on the metal surface, with molecules in such layers being chemically bound with each other and with the metal surface. The chemical bonds between NFHC molecules in the protective layer result from the formation of a polymeric complex compound with the metal cations formed in the course of corrosion. It is shown that it is more expedient to use not individual NFHCs but NFHC-containing mixtures. The reference list includes 260 references.
\end{abstract}

Keywords: acid corrosion, corrosion inhibitors, metal, steel, nitrogen-containing heterocyclic compounds, pyrrole, pyrrolidine, pyrrolidone, pyrazole, pyrazoline, imidazole, imidazoline, imidazolidine, triazole, tetrazole, indole, benzimidazole, benzotriazole.

Received: March 7, 2020. Published: April 6, 2021

doi: $\underline{10.17675 / 2305-6894-2020-10-2-2}$

\section{Introduction}

In recent decades, organic heterocyclic compounds, basically represented by nitrogencontaining heterocycles, are being actively studied as corrosion inhibitors (CIs) for ferrous and nonferrous metals [1-7] in aqueous (acidic [8-13], carbonate [14, 15], hydrogen sulfide-containing [16,17], neutral [18], alkaline [19]) and vapor-phase [20] media. Solutions of mineral and organic acids that find a wide application in various spheres of 
human activity are the most important media among those characterized by high corrosivity toward metals and alloys. These spheres include metallurgy, housing and utilities infrastructure, and oil and gas complex.

Mineral acids are used to strip scale from steels. Various acid formulations are applied for efficient removal of mineral scale and rust from the inner surfaces of various process equipment (including metallic heat exchangers). To stimulate the output of raw hydrocarbons and water, oil-, gas-, and water-bearing deposits are often treated with acid solutions. In the course of these operations, an acid contacts metallic parts of the equipment used in such processes. This results in degradation of metallic parts and an increase in acid consumption. The only acceptable method for protecting metals in such media is to use CIs, i.e., chemical compounds or formulations that, when present in the system in sufficient amounts, decrease the metal corrosion rate without any significant change in the concentration of any corrosive agent [21]. To date, a lot of new and important data have been published in scientific literature on the metal protection in acid media by nitrogen-containing heterocyclic compounds, which requires that these data be generalized, systematized, and analyzed in detail. This would allow one to determine the groups of such compounds that are most promising as CIs, elucidate the general regularities of the mechanism of their protective action, determine the ways of enhancing the protection efficiency, and formulate practical recommendations for the application of these CIs under real process conditions.

N-CONTAINING FIVE-COMPONENT HETEROCYCLIC COMPOUNDS

With one heteroatom
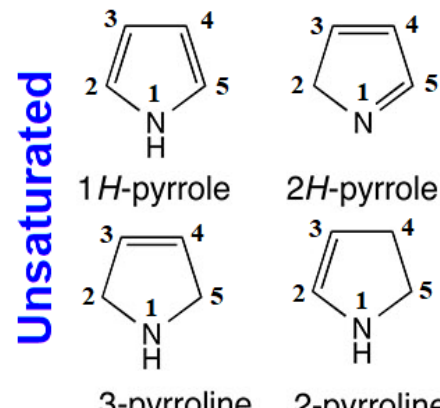

2H-pyrrole

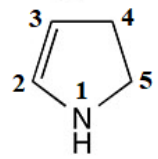

3-pyrroline 2-pyrroline

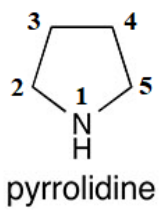

Figure 1. The most important nitrogen-containing five-membered organic heterocycles used as part of corrosion inhibitors.

Our earlier review [9] discusses in detail the options of metal protection in acid media by nitrogen-containing six-membered heterocycles. This review considers similar five- 
membered structures that encompass saturated and unsaturated simple compounds (Figure 1) and complex cyclic systems including such structures [22]. Let us state from the start that we have already analyzed the specifics of metal protection in acid media by nitrogen-containing five-membered heterocyclic compounds (NFHCs) [8]. However, the interest in these compounds, the constantly expanding choice of such compounds, and important results obtained in recent years in their study made it necessary to write this paper. Review [10] analyzes the specifics of steel protection in acid media by triazole derivatives, but the data presented there mainly cover the high-temperature corrosion conditions $\left(t \geq 80^{\circ} \mathrm{C}\right)$.

The chemical composition of steels discussed in this review is provided in Table 1 .

Table 1. Chemical composition of metals.

\begin{tabular}{|c|c|}
\hline Metal brand & Content elements in mass percent, $\%$ \\
\hline $\begin{array}{l}\text { Temper-rolled low- } \\
\text { carbon steel [23] }\end{array}$ & $\begin{array}{c}0.022 \mathrm{C} ; 0.004 \mathrm{Si} ; 0.14 \mathrm{Mn} ; 0.010 \mathrm{Cr} \text {; up to } 0.002 \mathrm{Ni} ; 0.015 \mathrm{Cu} ; 0.017 \mathrm{~S} \text {; } \\
\text { 0.007 P; remainder Fe }\end{array}$ \\
\hline Mild steel $[28,29]$ & $0.076 \mathrm{C} ; 0.192 \mathrm{Mn} ; 0.026 \mathrm{Si} ; 0.012 \mathrm{P} ; 0,050 \mathrm{Cr} ; 0.023 \mathrm{Al}$; remainder Fe \\
\hline Mild steel [31] & $0.21 \mathrm{C} ; 0.05 \mathrm{Mn} ; 0.01 \mathrm{Si} ; 0.09 \mathrm{P} ; 0,05 \mathrm{~S} ; 0.01 \mathrm{Al}$; remainder Fe \\
\hline Mild steel [33] & 0.14 C; 0.35 Mn; 0.17 Si; 0.025 S; 0.03 P; remainder Fe \\
\hline Carbon steel L-52 [46] & $0.26 \mathrm{C} ; 1,35 \mathrm{Mn} ; 0.05 \mathrm{~S} ; 0.04 \mathrm{P} ; 0.005 \mathrm{Nb} ; 0.02 \mathrm{~V} ; 0.03 \mathrm{Ti}$; remainder Fe \\
\hline C38 steel [47] & $\begin{array}{l}\text { 0.36 C; } 0.27 \mathrm{Si} ; 0.66 \mathrm{Mn} ; 0.21 \mathrm{Cr} ; 0.22 \mathrm{Cu} ; 0.02 \mathrm{~S} ; 0.015 \mathrm{P} ; 0.02 \mathrm{Mo} ; \\
\text { remainder Fe }\end{array}$ \\
\hline Mild steel [73] & $0.16 \mathrm{C} ; 0.032 \mathrm{Mn} ; 0.18 \mathrm{Si} ; 0.026 \mathrm{~S} ; 0.03 \mathrm{P}$; remainder Fe \\
\hline Steel P110 [76] & $\begin{array}{c}\text { 0.26 C; } 0.19 \mathrm{Si} ; 1,37 \mathrm{Mn} ; 0.004 \mathrm{P} ; 0.004 \mathrm{~S} ; 0.148 \mathrm{Cr} ; 0.28 \mathrm{Ni} ; 0.019 \mathrm{Cu} ; \\
\text { 0.013 Mo; 0.006 V; 0,026 Al; remainder Fe }\end{array}$ \\
\hline Copper [95] & $0.019 \mathrm{Al} ; 0.001 \mathrm{Ni} ; 0.116 \mathrm{Si} ; 0.004 \mathrm{Mn}$; remainder $\mathrm{Cu}$ \\
\hline Mild steel [105] & $0.20 \mathrm{C} ; 0.35 \mathrm{Mn} ; 0.16 \mathrm{Si} ; 0.01 \mathrm{~S} ; 0.05 \mathrm{P}$; remainder Fe \\
\hline Steel 3 & $\begin{array}{l}0.14-0.22 \mathrm{C} ; 0.15-0.33 \mathrm{Si} ; 0.40-0.65 \mathrm{Mn} \text {; up to } 0.3 \mathrm{Cr} \text {; up to } 0.30 \mathrm{Ni} \text {; up to } \\
0.008 \mathrm{~N} \text {; up to } 0.30 \mathrm{Cu} \text {; up to } 0.05 \mathrm{~S} \text {; up to } 0.04 \mathrm{P} \text {; up to } 0.08 \mathrm{As} \text {; remainder } \\
\qquad \mathrm{Fe}\end{array}$ \\
\hline Steel 20 & $\begin{array}{l}0.17-0.24 \mathrm{C} ; 0.17-0.37 \mathrm{Si} ; 0.35-0.65 \mathrm{Mn} \text {; up to } 0.25 \mathrm{Cr} \text {; up to } 0,30 \mathrm{Ni} \text {; up } \\
\quad \text { to } 0.30 \mathrm{Cu} \text {; up to } 0.04 \mathrm{~S} \text {; up to } 0.035 \mathrm{P} \text {; up to } 0.08 \mathrm{As} \text {; remainder } \mathrm{Fe}\end{array}$ \\
\hline Carbon steel [129] & $1.68 \mathrm{C} ; 0,65 \mathrm{~N} ; 0.10 \mathrm{Na} ; 0.07 \mathrm{Mg} ; 0.39 \mathrm{Cr} ; 0.69 \mathrm{Mn}$; remainder Fe \\
\hline 316 Stainless steel [139] & $\begin{array}{c}0.013 \mathrm{C} ; 0.3 \mathrm{Si} ; 1.4 \mathrm{Mn} ; 10.18 \mathrm{Ni} ; 0.001 \mathrm{~S} ; 0.026 \mathrm{P} ; 17.2 \mathrm{Cr} ; 2.1 \mathrm{Mo} ; 0.026 \\
\mathrm{~N} \text {; remainder Fe }\end{array}$ \\
\hline $\begin{array}{l}\text { 12Kh18N10T steel } \\
{[140,143]}\end{array}$ & $\begin{array}{l}\text { up to } 0.12 \mathrm{C} \text {; up to } 0.8 \mathrm{Si} \text {; up to } 2 \mathrm{Mn} \text {; } 9-11 \mathrm{Ni} \text {; up to } 0.02 \mathrm{~S} \text {; up to } 0.035 \mathrm{P} \text {; } \\
\qquad 17-19 \mathrm{Cr} \text {; up to } 0.3 \mathrm{Cu} \text {; up to } 0.5 \mathrm{Ti} \text {; remainder } \mathrm{Fe}\end{array}$ \\
\hline
\end{tabular}




\begin{tabular}{|c|c|}
\hline Metal brand & Content elements in mass percent, \% \\
\hline $\begin{array}{l}\text { 1Kh18N9T steel } \\
{[141,144]}\end{array}$ & 17.57 Cr; $9.16 \mathrm{Ni}$; $1.92 \mathrm{Fe}$; $0.83 \mathrm{Ti}$; $0.52 \mathrm{Si}$; remainder Fe \\
\hline 08Kh18N10T steel [142] & $\begin{array}{l}0.08 \mathrm{C} ; 0.8 \mathrm{Si} \text {; up to } 2 \mathrm{Mn} ; 9-11 \mathrm{Ni} \text {; up to } 0.02 \mathrm{~S} \text {; up to } 0.035 \mathrm{P} ; 17-19 \mathrm{Cr} \text {; } \\
\text { up to } 0.3 \mathrm{Cu} \text {; up to } 0.7 \mathrm{Ti} \text {; remainder } \mathrm{Fe}\end{array}$ \\
\hline Copper M1 & $\begin{array}{c}\text { Up to } 0.005 \mathrm{Fe} \text {; up to } 0.002 \mathrm{Ni} \text {; up to } 0.004 \mathrm{~S} \text {; up to } 0.002 \mathrm{As} \text {; up to } 0.005 \\
\mathrm{~Pb} \text {; up to } 0.004 \mathrm{Zn} \text {; up to } 0.05 \mathrm{O} \text {; up to } 0.002 \mathrm{Sb} \text {; up to } 0.001 \mathrm{Bi} \text {; up to } 0.002 \\
\mathrm{Sn} \text {; remainder } \mathrm{Cu}(99.90)\end{array}$ \\
\hline Brass L63 & $\begin{array}{l}\text { Up to } 0.2 \mathrm{Fe} \text {; up to } 0.001 \mathrm{P} \text {; up to } 0.07 \mathrm{~Pb} ; 34.5-38 \mathrm{Zn} \text {; up to } 0.005 \mathrm{Sb} \text {; up } \\
\text { to } 0.002 \mathrm{Bi} \text {; remainder } \mathrm{Cu}\end{array}$ \\
\hline Zinc Ts2 & $\begin{array}{l}\text { Up to } 0.05 \mathrm{Fe} \text {; up to } 0.005 \mathrm{Cu} \text {; up to } 0.01 \mathrm{As} \text {; up to } 1 \mathrm{~Pb} \text {; up to } 0.002 \mathrm{Sn} \text {; up } \\
\text { to } 0.2 \mathrm{Cd} \text {; remainder } \mathrm{Zn}\end{array}$ \\
\hline Aluminum D16 & $\begin{array}{l}\text { Up to } 0.5 \mathrm{Fe} \text {; up to } 0.5 \mathrm{Si} ; 0.3-0.9 \mathrm{Mn} \text {; up to } 0.1 \mathrm{Ni} \text {; up to } 0.1 \mathrm{Ti} ; 3.8-4.9 \\
\qquad \mathrm{Cu} ; 1.2-1.9 \mathrm{Mg} \text {; up to } 0.3 \mathrm{Zn} \text {; remainder } \mathrm{Al}\end{array}$ \\
\hline Aluminum AMg3,5 & $\begin{array}{l}\text { Up to } 0.4 \mathrm{Fe} \text {; up to } 0.25 \mathrm{Si} \text {; up to } 0.1 \mathrm{Mn} ; 0.15-0.35 \mathrm{Cr} \text {; up to } 0.2 \mathrm{Ti} \text {; up to } \\
0.1 \mathrm{Cu} \text {; up to } 0.0008 \mathrm{Be} ; 3.1-3.9 \mathrm{Mg} \text {; up to } 0.2 \mathrm{Zn} \text {; remainder } \mathrm{Al}\end{array}$ \\
\hline Mild steel Q235 [171] & 0.17 C; 0.47 Mn; 0.26 Si; 0.017 S; 0.0048 P; remainder Fe \\
\hline Steel N80 & $\begin{array}{c}0.34-0.38 \mathrm{C} ; 0.20-0.35 \mathrm{Si} ; 1.45-1.7 \mathrm{Mn} \text {; up to } 0.02 \mathrm{P} \text {; up to } 0.015 \mathrm{~S} \text {; up to } \\
0.15 \mathrm{Cr} ; 0.11-0.16 \mathrm{~V} \text {; remainder } \mathrm{Fe}\end{array}$ \\
\hline $\begin{array}{l}\text { Steel (V.E.W. Boehler, } \\
\text { Austria) [204] }\end{array}$ & $0.05 \mathrm{C} ; 0.6 \mathrm{Mn} ; 0.36 \mathrm{P} ; 0.03 \mathrm{~S}$; remainder Fe \\
\hline $\begin{array}{l}\text { Stainless steel SS304 } \\
\text { [207] }\end{array}$ & $\begin{array}{c}0.044 \mathrm{C} ; 0.30 \mathrm{Si} ; 0.8 \mathrm{Mn} ; 9.60 \mathrm{Ni} ; 0.0015 \mathrm{~S} ; 0.0025 \mathrm{P} ; 17.79 \mathrm{Cr} \text {; remainder } \\
\mathrm{Fe}\end{array}$ \\
\hline Carbon steel [210] & $0.28 \mathrm{C} ; 1.40 \mathrm{Mn} ; 0.03 \mathrm{~S} ; 0.03 \mathrm{P} ; 0.06 \mathrm{Ti}$; remainder Fe \\
\hline $\begin{array}{c}\text { Steel 70S2KhA } \\
{[232,233]}\end{array}$ & $0.7 \mathrm{C} ; 0.52 \mathrm{Mn} ; 1.52 \mathrm{Si} ; 0.3 \mathrm{Cr}$; remainder Fe \\
\hline
\end{tabular}

\section{Inhibition of metal corrosion in acid media by nitrogen-containing five-membered heterocyclic compounds}

\section{Simple cyclic systems}

The simplest aromatic NFHC is pyrrole that is characterized by high chemical reactivity. Using pyrrole as a CI of acid corrosion is of little promise since it is unstable in acid solutions: protons catalyze its polymerization to give an insoluble red resinous product [22]. Despite this, there are data on the protection of temper rolled low-carbon steel by this compound $(0.1 \%)$ in $2 \mathrm{~N} \mathrm{H}_{2} \mathrm{SO}_{4}\left(38-93^{\circ} \mathrm{C}\right)$ [23]. As it could be expected, the efficiency of the CI is low and the inhibitor's protective effect $(Z)$ is $76-79 \% . Z=95-97$ and $97-99 \%$ only in the presence of $17 \mathrm{mM} \mathrm{KCl}$ and $\mathrm{KI}$, respectively, though these salts provide $Z=4$ 22 and $97 \%$ by themselves. 
Substituted pyrroles (2,5-difuryl-N-methylpyrrole [24], 1-phenyl-1H-pyrrole-2,5-dione, 1-(4-methylphenyl)-1H-pyrrole-2,5-dione [25], 1-(1-benzyl-4-(4-chlorophenyl)-2,5dimethyl-1H-pyrrol-3-yl)ethanone [26]) protect different steels in cold $1 \mathrm{M} \mathrm{HCl} . \mathrm{N}-((1 \mathrm{H}$ pyrrol-2-yl)methylene)aniline, $\quad N$-((1H-pyrrol-2-yl)methylene)-4-methylaniline, $\quad N-((1 H$ pyrrol-2-yl)methylene)-4-methoxyaniline, and $\quad N$-((1H-pyrrol-2-yl)methylene)-3,5dimethylaniline at a content of $500-2000 \mathrm{ppm}$ in hot $10 \% \mathrm{HCl}\left(60^{\circ} \mathrm{C}\right)$ considerably inhibit mild steel corrosion, providing $Z=95.8-99.6 \%$ [27]. In $1 \mathrm{M} \mathrm{HCl}\left(35^{\circ} \mathrm{C}\right), 2$-amino-3-methyl3-phenyl-5-(phenylthio)-3H-pyrrole-4-carbonitrile, 2-amino-3-(4-hydroxyphenyl)-3-methyl5-(phenylthio)-3H-pyrrole-4-carbonitrile, 2-amino-3-(2,4-dihydroxyphenyl)-3-methyl-5(phenylthio)-3H-pyrrole-4-carbonitrile [28], and 2-amino-3-methyl-3-(4-nitrophenyl)-5(phenylthio)-3H-pyrrole-4-carbonitrile [29] at a content of $50 \mathrm{mg} / \mathrm{L}$ slow down the corrosion of mild steel considerably $(Z=94.4-98.7 \%)$, but their protective effect decreases with an increase in temperature $(t)$.

Low-molecular pyrrolidine derivatives weakly suppress iron corrosion in $6.1 \mathrm{M} \mathrm{HCl}$ $\left(30^{\circ} \mathrm{C}\right)$. According to voltammetric data, the protective effect of CIs grows in a series of $\mathrm{N}$ methylpyrrolidine < N-ethylpyrrolidine < pyrrolidine. Besides, $Z=60 \%$ in the presence of $10 \mathrm{mM}$ pyrrolidine in a solution [30]. In $0.5 \mathrm{M} \mathrm{H}_{2} \mathrm{SO}_{4}\left(25^{\circ} \mathrm{C}\right), 1$-R-pyrrolidine-2-one $(R=$ $-\mathrm{CH}=\mathrm{CH}_{2},-\left(\mathrm{CH}_{2}\right)_{2}-\mathrm{S}-\left(\mathrm{CH}_{2}\right)_{2}-\mathrm{OH},-\left(\mathrm{CH}_{2}\right)_{2}-\mathrm{S}-\mathrm{CH}_{2}-\mathrm{COOH}[31],-\left(\mathrm{CH}_{2}\right)_{2}-\mathrm{P}(\mathrm{O})\left(\mathrm{OC}_{2} \mathrm{H}_{5}\right)_{2}$ [32]) also weakly inhibits steel corrosion $\left(Z_{\max }=71-89 \%\right)$. Despite the presence of a bulky substituent in the pyrrolidine ring in 1-((pyridin-2-ylamino)(pyridin-4-yl)methyl)pyrrolidine-2,5-dione, it is also inefficient in the inhibition of mild steel corrosion in $1 \mathrm{M}$ $\mathrm{HCl}$ [33]. Only quaternized salts ( $N, N$-dipropargylpyrrolidium bromide, $N$-dodecyl- $N$ propargylpyrrolidium bromide, and $N$-hexadecyl- $N$-propargylpyrrolidium bromide (25$50 \mathrm{ppm})$ ) can efficiently protect low-carbon steel in $1 \mathrm{M} \mathrm{HCl}$ at $25^{\circ} \mathrm{C}(Z \geq 95 \%)$ [34]. The observed effect is largely determined by the presence in these compounds of a propargyl moiety that is very reactive and can cause polymerization of CI molecules on the metal surface to form a protective polymer layer [8,35-37]. Ammonium and sodium pyrrolidine dithiocarboxylates that provide $Z$ up to $90 \%$ were studied in $0.5 \mathrm{M} \mathrm{HCl}$ and $5 \% \mathrm{HCl}$ as CIs for copper and Monel (composition, wt.\%: $70 \mathrm{Ni}$ and $30 \mathrm{Cu}$ ) [38, 39]. Vinylpyrrolidonebased polymer and copolymer additives are used for the protection of mild and carbon steels in $\mathrm{HCl}$ and $\mathrm{H}_{2} \mathrm{SO}_{4}$ solutions [40-45], but even though these compounds provide efficient metal protection in cold acid solutions, they fail to do so upon temperature increase.

The scope of five-membered nitrogen-containing CIs with two heteroatoms is more extensive. Imidazole itself weakly suppresses steel corrosion [46-48]. According to voltammetric data, the addition of $5 \mathrm{mM}$ imidazole provides only $Z=83 \%$ for carbon steel L-52 in $0.5 \mathrm{M} \mathrm{H}_{2} \mathrm{SO}_{4}\left(30^{\circ} \mathrm{C}\right)$ [46]. In $1 \mathrm{M} \mathrm{HCl}$ in the presence of $4 \%$ imidazole, $Z=60 \%$ for steel C38 [47]. Similarly, imidazoles with small substituents fail to provide good protection of steels in $\mathrm{HCl}$ solutions [49-51]. In contrast, imidazoles with four phenyl substituents: 

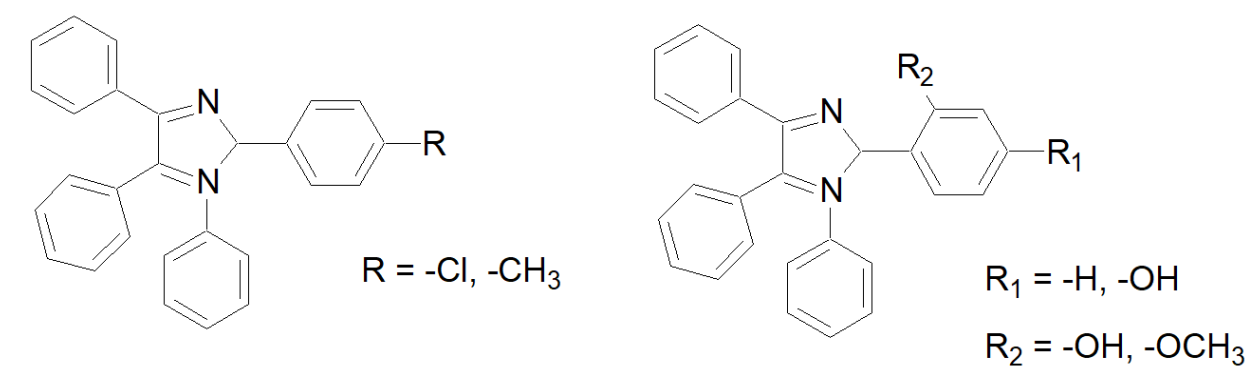

provide efficient corrosion inhibition of low-carbon steel in $1 \mathrm{M} \mathrm{HCl}$ and $0.5 \mathrm{M} \mathrm{H}_{2} \mathrm{SO}_{4}$ $[52,53]$. In recent years, imidazole derivatives with quaternized nitrogen atoms in the heterocycle - Gemini surfactants [54], imidazole zwitterions [55-57], and ionic liquids [5867] have been actively used as steel CIs in acids. Imidazole derivatives are considered as CIs for nonferrous metals: copper in $\mathrm{H}_{2} \mathrm{SO}_{4}[68,69], \mathrm{HCl}$ [70] and $\mathrm{HNO}_{3}$ [71], aluminum in $\mathrm{HCl}$ [72].

As opposed to compounds containing an aromatic imidazole ring, imidazoline [73-79] and imidazolidine [80] derivatives are less studied as CIs. Specifically, 3,4dimethoxyphenyl-2-imidazoline $(40 \mathrm{ppm})$ on low-carbon steel in $0.5 \mathrm{M} \mathrm{H}_{2} \mathrm{SO}_{4}\left(30^{\circ} \mathrm{C}\right)$ provides $Z=58.3 \%$ according to the data of three-hour tests. The best result is observed with 4-methoxyvinyl-2-imidazoline, where $Z=82.4 \%$ at a lower content in the corrosion medium (20 ppm) [73]. Addition of $0.4 \mathrm{~g} / \mathrm{L}$ 2-heptadecyl-1-[2-(octadecanoylamino)ethyl]-2imidazoline poorly protects steel St37 (Erdemir Steel Co., Turkey) in $15 \% \mathrm{HCl}\left(25^{\circ} \mathrm{C}\right)$, as shown by electrochemical impedance spectroscopy (EIS) data: $Z=47.9 \%$ [74]. Under similar conditions, $Z=89.9 \%$ for $N$-(2-(2-tridecyl-4,5-dihydro-1H-imidazol-1yl)ethyl)tetradecanamide [75]. Of interest is 2-methyl-4-phenyl-1-tosyl-4,5-dihydro- $1 \mathrm{H}$ imidazole that provides $Z=94.6 \%$ at a content of $0.3 \mathrm{~g} / \mathrm{L}$ in $1 \mathrm{M} \mathrm{HCl}\left(60^{\circ} \mathrm{C}\right)$ according to the data of 72-hour tests on carbon steel P110 [76]. A high-molecular imidazolidine derivative, 3-methyl-5,5'-diphenylimidazolidine-2,4-dione, poorly inhibits mild steel corrosion according to the data of gravimetric studies in $1 \mathrm{M} \mathrm{HCl}\left(25^{\circ} \mathrm{C}\right)[80]$.

Pyrazole that is an aromatic system is an inefficient CI. According to voltammetric data, iron $(99.995 \%)$ corrosion in $1 \mathrm{M} \mathrm{HCl}\left(25^{\circ} \mathrm{C}\right)$ containing $10 \mathrm{mM}$ pyrazole is inhibited only 3.4-fold [81]. Using pyrazole derivatives with various substituents, good steel protection cab be provided, predominantly in $\mathrm{HCl}$ solutions [82-92] and, more rarely, in $\mathrm{H}_{2} \mathrm{SO}_{4}$ solutions [93]. Pyrazole derivatives can be used for the protection of nonferrous metals in acid media. 5-Amino-3-[4'-chlorophenyl]pyrazole and 5-amino-3-[4'-methylphenyl]pyrazole in $0.06 \mathrm{M}$ $\mathrm{HCl}\left(25^{\circ} \mathrm{C}\right)$ provide $Z=89 \%$ for zinc [94]. Copper corrosion in $2 \mathrm{M} \mathrm{HNO}_{3}\left(25^{\circ} \mathrm{C}\right)$ is inhibited almost 10 -fold in the presence of $11 \cdot 10^{-6} \mathrm{M} 3^{\prime}$-(3,4-dichlorophenyl)-2',3',6,7,8,9hexahydro-2'-phenyl-5'-styrylspiro[benzocyclo-heptene-6(5H),4'(4H-pyrazol)]-5-one, but an increase in the corrosion medium temperature weakens the protective effects [95]. Derivatives of a more saturated pyrazoline ring were also studied as steel CIs in acids [96100].

Active studies of triazoles as CIs of metals in acid media were covered in recent review papers $[101,102]$ that are largely a collection of reference data. Substituted 1,2,3-triazoles 
[103-109] and 4,5-dihydro-1H-1,2,3-triazole [110] are predominantly used for the protection of low-carbon and carbon steels in $\mathrm{HCl}$ solutions. Of interest is $(2 R)$-benzyl-2amino-3-((1-(( $(2 R, 3 R, 4 S, 5 S, 6 S)-3,4,5$-tris(benzyloxy)-6-methoxytetrahydro-2H-pyran-2yl)methyl)-1H-1,2,3-triazol-4-yl)methoxy) butanoate that provides $Z=97.0-99.4 \%$ in $2-$ $6 \mathrm{M} \mathrm{HCl}$ at $25^{\circ} \mathrm{C}[105]$.

1,2,4-Triazoles are studied better. 3,5-Bis $(R)$-4-amino-1,2,4-triazole $(\mathrm{R}=4$ methoxyphenyl [111], $m$-tolyl [112], methoxy [113], 2-thienylmethyl [114], pyridyl [115], 4-chlorophenyl [116], or 3,4-dimethoxyphenyl [117]) are used to protect mild steel in $\mathrm{HCl}$ and $\mathrm{H}_{3} \mathrm{PO}_{4}$ solutions. Azomethines with various structures synthesized on the basis of 4amino-1,2,4-triazole [118-122] and 3-amino-1,2,4-triazole derivatives [123] are mild steel corrosion inhibitors in $\mathrm{HCl}$ solutions. Earlier [124], we pointed out the high efficiency of azomethines containing moieties of cinnamic aldehyde and a nitrogen-containing heterocycle in the inhibition of steel corrosion in hot $\left(t \leq 105^{\circ} \mathrm{C}\right) \mathrm{HCl}$ solutions. Indeed, in $2 \mathrm{M} \mathrm{HCl}\left(20-100^{\circ} \mathrm{C}\right)$, the addition of $5 \mathrm{mM} N$-cinnamylidene- $1 \mathrm{H}-1,2,4$-triazole-3-amine suppresses the corrosion of steel 3 by a factor of 5.1-278 [125]. With an increase in $t$, the protective effect of this compound is only enhanced, which allows one to consider it as a high-temperature CI $[36,126]$. Under similar conditions, the additive of the well-known CI, cinnamic aldehyde [127] used in the synthesis of this compound, inhibits corrosion only 4.2-24 fold. Moreover, the temperature maximum of its efficiency is at $90^{\circ} \mathrm{C}$, which is a poorer result [125]. Parallel studies of 3-amino-1,2,4-triazole showed its weak inhibitive properties $(\gamma \leq 2.3)$.

1,2,4-Triazole derivatives with bulky substituents are characterized by high efficiency in steel protection in $\mathrm{HCl}$ solutions. A Gemini surfactant, 3,5-bis(methylene octadecyl dimethylammonium chloride)-1,2,4-triazole $(1 \mathrm{mM})$, in $1 \mathrm{M} \mathrm{HCl}$ provides $Z=98.3 \%$ for carbon steel at $25^{\circ} \mathrm{C}$ [128]. High-molecular compounds based on 1,2,4-triazole often contain sulfur $[129,130]$. In the presence of $1 \mathrm{mM}$ 5-hexylsulfanyl-1,2,4-triazole, $12 \mathrm{~h}$ exposure of a carbon steel sample in this medium provides $Z=93 \%$ [129].

The capabilities of 1,2,4-triazole derivatives in preventing steel corrosion in acid media are unique to a large extent. In fact, 3-substituted 1,2,4-triazole derivatives with various structures (inhibitors such as IFKhAN-92 [131-133], IFKhAN-93 [134], IFKhAN-94 [135], as well as IFKhAN-96 that is a 4-substituted 1,2,4-triazole [136]) inhibit the corrosion of mild steels in $\mathrm{HCl}$ and $\mathrm{H}_{2} \mathrm{SO}_{4}$ solutions at $t$ up to $100^{\circ} \mathrm{C}$ in many cases [10]. They demonstrate a protective effect in a wide range of acid concentrations, $C=1-8 \mathrm{M}$ [134-136]. At $t=60^{\circ} \mathrm{C}$, the addition of $5 \mathrm{mM}$ IFKhAN-94 inhibits the corrosion of steel 3 by a factor of 24.8-121 in $1-8 \mathrm{M} \mathrm{HCl}$ and 32.9-905 in 1-8 $\mathrm{M} \mathrm{H}_{2} \mathrm{SO}_{4}$ [135]. It is important for practical purposes that while triazole derivatives efficiently inhibit steel corrosion, they do not slow down the removal of thermal scale in acids [137]. It has been shown for CIs IFKhAN-92 and IFKhAN-93 as examples [138] that triazole derivatives efficiently suppress steel hydrogenation in addition to considerable inhibition of its corrosion in $\mathrm{HCl}$ and $\mathrm{H}_{2} \mathrm{SO}_{4}$ solutions.

Chromium-nickel stainless steels are unstable in (especially hot) $\mathrm{HCl}$ and $\mathrm{H}_{2} \mathrm{SO}_{4}$ solutions. There are problems regarding their protection by inhibitors in these media, 
including the protection by individual triazoles. In fact, at $2000 \mathrm{ppm}, 4-\{[4-$ (dimethylamino)benzylidene]amino -5-methyl-4H-1,2,4-triazole-3-thiol in $2 \mathrm{M} \mathrm{HCl} \mathrm{(30-}$ $50^{\circ} \mathrm{C}$ ) provides $Z=69.8-91.8 \%$ on stainless steel 316 according to voltammetric data [139]. Of more interest is IFKhAN-92 that considerably inhibits the corrosion and hydrogenation of chromium-nickel steels $(12 \mathrm{Kh} 18 \mathrm{~N} 10 \mathrm{~T}, 1 \mathrm{Kh} 18 \mathrm{~N} 9 \mathrm{~T}, 08 \mathrm{Kh} 18 \mathrm{~N} 10 \mathrm{~T})$ at $C=5 \mathrm{mM}$ in hot $\mathrm{HCl}$ solutions, including those containing $\mathrm{H}_{2} \mathrm{~S}$ [140-142]. Steel protection by IFKhAN-92 in $\mathrm{H}_{2} \mathrm{SO}_{4}$ solutions is considerably enhanced in the presence of $\mathrm{H}_{2} \mathrm{~S}[143,144]$.

1,2,4-Triazole derivatives protect not only ferrous but also nonferrous metals in acid media. In $\mathrm{HCl}$ solutions, the addition of 4-amino-5-alkyl-3-mercapto-1,2,4-triazoles (alkyl = methyl, ethyl, propyl) inhibits the corrosion of copper (99.9\%) [145] and Muntz metal $(60 \% \mathrm{Cu}, 40 \% \mathrm{Zn})$ [146]. 3,5-Bis(4-methylphenyl)-4-amino-1,2,4-triazole and 3,5-bis(4methoxyphenyl)-4-amino-1,2,4-triazole protect copper (99.9\%) in $1 \mathrm{M} \mathrm{HCl}\left(30^{\circ} \mathrm{C}\right)$ [147]. IFKhAN-92 protects a wide range of nonferrous metals in mineral acids [148]. For copper $\mathrm{M} 1$ in $2 \mathrm{M} \mathrm{HCl}$ (room temperature) at $C_{\text {in }}=5 \mathrm{mM}$ and exposure duration of 7 days, $Z=97.7 \%$. In $2 \mathrm{M} \mathrm{H}_{2} \mathrm{SO}_{4}$ and $\mathrm{HCl}\left(95^{\circ} \mathrm{C}\right)$ for brass $\mathrm{L} 63$ at the same $C_{\text {in }}, Z=78$ and $86 \%$. Here, the inhibitor prevents the dezincification of the metal surface that is observed in the background media. In $2 \mathrm{M} \mathrm{H}_{2} \mathrm{SO}_{4}, \mathrm{HCl}$, and $\mathrm{H}_{3} \mathrm{PO}_{4}\left(25^{\circ} \mathrm{C}\right)$, the corrosion rates $(k)$ of zinc Ts2 are 753,1429 , and $121 \mathrm{~g} /\left(\mathrm{m}^{2} \cdot \mathrm{h}\right)$, respectively, against only $3.1,3.0$, and $4.6 \mathrm{~g} /\left(\mathrm{m}^{2} \cdot \mathrm{h}\right)$ in the presence of $5 \mathrm{mM}$ IFKhAN-92. In $2 \mathrm{M} \mathrm{HCl}\left(25^{\circ} \mathrm{C}\right)$, the $k$ of aluminum alloys D16 and $\mathrm{AMg} 3.5$ are 1425 and $97.2 \mathrm{~g} /\left(\mathrm{m}^{2} \cdot \mathrm{h}\right)$ against 3.0 and $0.7 \mathrm{~g} /\left(\mathrm{m}^{2} \cdot \mathrm{h}\right)$ in the presence of $10 \mathrm{mM}$ IFKhAN-92.

In $1 \mathrm{M} \mathrm{HCl}\left(25^{\circ} \mathrm{C}\right)$, tetrazole $(2 \mathrm{mM})$ stimulates the corrosion of pure iron $(99.96 \%)$ by accelerating the anodic and especially cathodic reactions on the metal, but its substituted derivative, 1-phenyl-5-mercapto-1,2,3,4-tetrazole, provides $Z=98.2 \%$ [149]. Substituted tetrazoles also suppress steel corrosion in $\mathrm{HCl}$ solutions [150-153]. Tetrazole derivatives (organic cations [154-159] and thiols $[160,161]$ ) were studied in $\mathrm{H}_{2} \mathrm{SO}_{4}$ solutions as steel CIs. Tetrazoles can protect steels in a wide temperature range. Addition of $5 \mathrm{mM} \mathrm{IFKhAN-}$ 95 (a 5-substituted tetrazole) in $2 \mathrm{M} \mathrm{H}_{2} \mathrm{SO}_{4}$ and $\mathrm{HCl}\left(25-95^{\circ} \mathrm{C}\right)$ inhibits the corrosion of steel 3 by a factor of 35-149 and 19-111, respectively. The maximum CI efficiency for $\mathrm{H}_{2} \mathrm{SO}_{4}$ is at $80^{\circ} \mathrm{C}$ and is not achieved for $\mathrm{HCl}$ under experimental conditions, which is characteristic of high-temperature CIs [162]. Organic tetrazole-based cations inhibit steel corrosion in acetic acid solutions [163] and in dilute solutions of chloroacetic acids $\left(\mathrm{ClCH}_{2} \mathrm{COOH}, \mathrm{Cl}_{2} \mathrm{CHCOOH}\right.$, and $\left.\mathrm{Cl}_{3} \mathrm{CCOOH}\right)$ [164]. Tetrazole-containing compounds are inhibitors of copper corrosion in solutions of $\mathrm{HCl}[165,166], \mathrm{H}_{2} \mathrm{SO}_{4}[167,168]$, and $\mathrm{HNO}_{3}$ [169], and of aluminum corrosion in $1 \mathrm{M} \mathrm{HCl} \mathrm{[170].}$

\section{Complex systems}

The simplest fused system containing a pyrrole moiety is indole that significantly inhibits the corrosion of mild steel Q235 in $0.1 \mathrm{M} \mathrm{H}_{2} \mathrm{SO}_{4}(Z=93.7-97.2 \%)$ at $C_{\mathrm{in}}=4-8 \mathrm{mM} \mathrm{[171].}$ In $1 \mathrm{M} \mathrm{H}_{2} \mathrm{SO}_{4}, 5$-aminoindole and 5-chloroindole provide weaker corrosion inhibition of steel than indole [172]. The efficiency of the protection of pure iron (Puratronic, 99.9999\%) 
in $1 \mathrm{M} \mathrm{HCl}\left(25^{\circ} \mathrm{C}\right)$ by nitrogen-containing heterocycles decreases in the series indole > benzotriazole > benzothiazole > benzoimidazole [173]. The corrosion of mild steels in acids is inhibited by indole-containing amines [174], azomethines [175], and ionic liquids [176]. Indole carboxylic acids were studied as CIs for mild steel in solutions of $\mathrm{HCl}$ [177] and $\mathrm{H}_{2} \mathrm{SO}_{4}$ [178] and for copper in $0.5 \mathrm{M} \mathrm{H}_{2} \mathrm{SO}_{4}$ [179]. More complex substituted cyclic systems, such as 3,6-dibromo-9-phenylcarbazole, 3(9H-carbazol-9-yl)-1,2-propanediol [180], 2,3,4,9-tetrahydro- $1 H$-carbazole-8-carboxylic acid, 6-methyl-2,3,4,9-tetrahydro- $1 H$ carbazole, 9-methyl-9H-carbazole-3-carboxylic acid [181], 9H-pyrido[3,4-b]indole, 1methyl-9H-pyrido[3,4-b]indole [182], and 1,4-bis(2-pyridyl)-5H-pyridazino[4,5-b]indole [183] were studied as mild steel CIs in cold $1 \mathrm{M} \mathrm{HCl} . Z>90 \%$ was observed in the $C_{\text {in }}$ range studied only with the latter compound. Two indolizine derivatives are of interest:
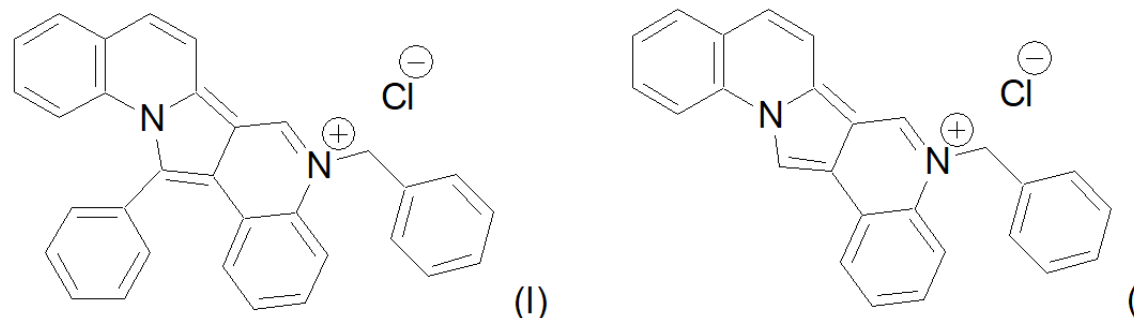

They protect steel $\mathrm{N} 80$ in $15-20 \% \mathrm{HCl}$ and $12 \% \mathrm{HCl}+3 \% \mathrm{HF}\left(90^{\circ} \mathrm{C}\right)$ where the background steel corrosion rates are $k_{0}=1243-1648$ and $1105 \mathrm{~g} /\left(\mathrm{m}^{2} \cdot \mathrm{h}\right)$, respectively [184]. In the presence of $1 \%$ compound (I), $k=2.0-10.6$ and $2.1 \mathrm{~g} /\left(\mathrm{m}^{2} \cdot \mathrm{h}\right)$, while in the presence of compound (II), $k=3.2-12.2$ and $2.1 \mathrm{~g} /\left(\mathrm{m}^{2} \cdot \mathrm{h}\right)$. With a $t$ increase to $120^{\circ} \mathrm{C}$, the protective effect of these compounds deteriorates, especially in $20 \% \mathrm{HCl} \mathrm{[185].}$

Individual benzimidazole (BI) manifests a weak protective effect in low-carbon steel protection in $1 \mathrm{M} \mathrm{HCl}\left(20^{\circ} \mathrm{C}\right)$. 2-Substituted BIs are more efficient. At $C_{\mathrm{in}}=1 \mathrm{mM}$, steel corrosion inhibition is enhanced in the series BI $<2$-methyl-BI $<2$-hydroxymethyl-BI $<2$ amino-BI $<2$-acetonitrile-BI $<2$-mercapto-BI. For the most efficient CIs, namely 2-aminoBI, 2-acetonitrile-BI, and 2-mercapto-BI, the maximum degrees of protection observed in hydrochloric acid are $Z_{\max }=94.0,94.0$, and $94.7 \%$ [186]. However, the $Z$ of even for the best of these CIs, 2-mercapto-BI (1 mM), decreases from 97 to $88 \%$ for mild steel with an increase in $t$ from 40 to $80^{\circ} \mathrm{C}$ in the same medium [187]. In many cases, BIs with bulky substituents of various nature [188-196] and ionic liquids on its basis [197,198] are promising for steel protection in $\mathrm{HCl}$ solutions. Quaternized BIs protect mild steel in $0.5 \mathrm{M}$ $\mathrm{H}_{2} \mathrm{SO}_{4}\left(25-55^{\circ} \mathrm{C}\right)$ [199] and a $6061 \mathrm{Al}-15$ vol.\% $\mathrm{SiC}_{(\mathrm{p})}$ composite in $0.1 \mathrm{M} \mathrm{HCl}$ and $\mathrm{H}_{2} \mathrm{SO}_{4}$ $\left(30-50^{\circ} \mathrm{C}\right)[200]$. Substituted derivatives of imidazo[1,2-a]pyridine

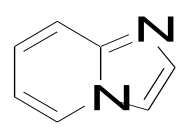

inhibit steel corrosion in a wide range of mineral acids $\left(\mathrm{HCl}, \mathrm{H}_{2} \mathrm{SO}_{4}, \mathrm{H}_{3} \mathrm{PO}_{4}\right)[6,201]$. 
Derivatives of pyrazolo-pyrimidine<smiles>c1ncc2[nH]ncc2n1</smiles>

were studied as corrosion inhibitors for pure copper in $0.5 \mathrm{M} \mathrm{H}_{2} \mathrm{SO}_{4}[202,203]$. The most efficient one, 4-mercapto-pyrazolo-pyrimidine, provides $Z=91 \%$ at $C_{\mathrm{in}}=0.5 \mathrm{mM}$ [203].

1,2,3-Benzotriazole (BTA) widely used as a CI [2-4] was also studied in acid media $[204,205]$. However, even in dilute $0.1 \mathrm{M} \mathrm{H}_{2} \mathrm{SO}_{4}\left(30^{\circ} \mathrm{C}\right), 0.01-0.09 \mathrm{M}$ BTA weakly inhibits mild steel corrosion, $Z=76.9-89.2 \%$ [204]. In the presence of 0.01 M BTA, an increase in $t$ from 30 to $65^{\circ} \mathrm{C}$ causes a decrease in $Z$ from 76.9 to $33.5 \%$. Addition of $\mathrm{CuSO}_{4}$ that enhances the protective effect of BTA can provide high $Z=99.4 \%\left(0.01 \mathrm{M} \mathrm{BTA}\right.$ with $\left.0.001 \mathrm{M} \mathrm{CuSO}_{4}\right)$ at $t=30^{\circ} \mathrm{C}$. A mixture of BTA with $\mathrm{Cu}$ (II) is more efficient in iron protection in $0.5 \mathrm{M} \mathrm{H}_{2} \mathrm{SO}_{4}$ $\left(30^{\circ} \mathrm{C}\right)$ [205]. The practical application of CI mixtures containing $\mathrm{Cu}$ (II) salts for steel protection can be dangerous. A reaction of $\mathrm{Cu}$ (II) cations with the metal results in the formation of metallic copper on its surface and appearance of $\mathrm{Cu}$-steel galvanic couples. This may result in corrosion localization in acid media and may adversely affect the further operation of steel structures. A mixture of BTA with disodium ethylenediamine tetraacetate is used for the protection of cold rolled steel in dilute $0.1 \mathrm{M} \mathrm{HCl}\left(30^{\circ} \mathrm{C}\right)$ [206].

According to voltammetric data, BTA $(10 \mathrm{mM})$ protects stainless steel SS304 in $0.1 \mathrm{M}$ $\mathrm{H}_{2} \mathrm{SO}_{4}\left(30-70^{\circ} \mathrm{C}\right)$, providing $Z=86.7-99.1 \%$. Importantly, an increase in $t$ results in a systematic increase in $Z$ [207]. Unsatisfactory results were obtained in the protection of low carbon steel in cold $1 \mathrm{M} \mathrm{HCl}$ and $1 \mathrm{M} \mathrm{HCl}+3.5 \% \mathrm{NaCl}$ by $100 \mathrm{ppm} 5$-methyl- $1 \mathrm{H}$ benzotriazole, whose $Z$ does not reach $90 \%$ even in combination with KI, a well known synergetic additive $(5 \mathrm{mM})$. BTA derivatives with bulkier substituents are efficient in corrosion inhibition of Armco iron and mild steel in $\mathrm{H}_{2} \mathrm{SO}_{4}$ solutions [208, 209]. An ionic liquid, 1-dodecyl-5-methyl- $1 H$-benzo[d][1,2,3]triazol-1-ium bromide, strongly inhibits carbon steel corrosion in cold $7 \mathrm{M} \mathrm{H}_{3} \mathrm{PO}_{4}$. However, for $1 \mathrm{mM}$ of this compound, an increase in $t$ from 20 to $80^{\circ} \mathrm{C}$ causes a decrease in $Z$ from 97.2 to $87.8 \%$ [210]. BTA and its derivatives were studied as corrosion inhibitors of copper and its alloys in $\mathrm{HCl}, \mathrm{H}_{2} \mathrm{SO}_{4}$, and $\mathrm{HNO}_{3}$ solutions [211-217]. Among the compounds studied, 1-(phenylsulfonyl)-1H-benzotriazole [211] and benzotriazole-1-carboxamide [212] are of interest as that provide $Z=93.5$ and $92.4 \%$ for copper, respectively, even at relatively low concentrations $(5 \mathrm{mM})$ in corrosive $1 \mathrm{M} \mathrm{HNO}_{3}\left(25^{\circ} \mathrm{C}\right)$. In $0.5 \mathrm{M} \mathrm{HCl}\left(30^{\circ} \mathrm{C}\right)$, copper corrosion is inhibited 27.5 -fold at a high content (50 mM) of $N$-(2-thiazolyl)-1H-benzotriazole-1-carbothioamide [213].

The compound with a complex structure

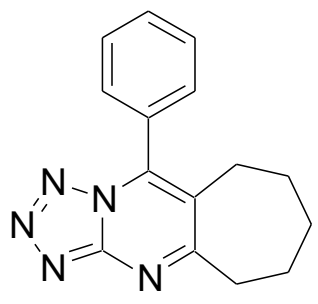


is a weak CI for low-carbon steel in $0.5 \mathrm{M} \mathrm{H}_{3} \mathrm{PO}_{4}$ that provides only $Z=71.5 \%$ at $C_{\text {in }}=0.1 \mathrm{M}$ [218].

Thus, a rather wide range of NFHCs including both simple and more complex systems have been studied as CIs of metals in acid media. These compounds are mainly recommended for the corrosion inhibition of various steels, though it is pointed out that they can be used for protection of nonferrous metals, chiefly copper and copper-based alloys. The effect of these CIs is manifested in solutions of $\mathrm{HCl}, \mathrm{H}_{2} \mathrm{SO}_{4}, \mathrm{H}_{3} \mathrm{PO}_{4}$, and, in some cases, even $\mathrm{HNO}_{3}$. Unfortunately, no systematic studies on the effect of the structure of NFHCs on their protection from metal corrosion in acid media are provided in scientific literature. However, even the available data allow us to draw the conclusion that the efficiency of the heterocyclic systems under consideration increases when bulky substituents are introduced into their structure. However, this is not always the case.

It is worthy of note that many of the compounds considered as CIs in this review are difficult to obtain and expensive, which considerably limits the prospects of their industrial application. Besides, industrial acid treatment conditions imply that they should be used at elevated temperatures. As the data on metal protection by CIs provided here either correspond to cold solutions or demonstrate a decrease in protection with an increase in $t$, only some of the compounds are of particular interest. For example, CIs of the IFKhAN series can enhance the protective effect in steel corrosion up to temperatures close to $100^{\circ} \mathrm{C}$, which is promising for their industrial use. The efficiency of the CIs under consideration can be enhanced by chemical modification of their molecular structure, which significantly increases their cost. This way is of little promise. An alternative is to develop mixtures with other compounds based on the already studied heretocyclic CIs, which does not lead to any considerable rise in their cost, expands the range of their application, and decreases their consumption. Certain important results in this direction have already been achieved, as described in the next section.

\section{Composite CIs based on nitrogen-containing five-membered heterocyclic compounds}

This section considers the most outstanding results in metal protection in acid media by composite NFHC-based CIs. A conventional method of enhancing the protective effect of organic CIs is to develop binary formulations with metal halides on their basis [219]. Indeed, binary formulations such as IFKhAN-92 + KI and IFKhAN-92 + KBr can protect steel 20 in $\mathrm{H}_{2} \mathrm{SO}_{4}$ solutions at $t \leq 200^{\circ} \mathrm{C}[133,220]$. The application of the IFKhAN-92 + urotropine mixture in $\mathrm{HCl}$ solutions allows one to significantly inhibit the corrosion of steel 20 at $t \leq 160^{\circ} \mathrm{C}[132,220]$. A more interesting result is provided by a three-component mixture of IFKhAN-92 + KI + urotropine that protects not only low-carbon, but also, most notably, chromium-nickel steels $[142,221,222]$ under high-temperature acid corrosion conditions $\left(t>100^{\circ} \mathrm{C}\right)$. Importantly, it is sufficient to use binary formulations of IFKhAN-92 $+\mathrm{KI}$, IFKhAN-92 $+\mathrm{KBr}\left(\mathrm{H}_{2} \mathrm{SO}_{4}\right.$ solutions), and IFKhAN-92 + urotropine $(\mathrm{HCl}$ solutions $)$ in acid solutions with $t \leq 100^{\circ} \mathrm{C}$ for the protection of chromium-nickel steels [140, 141, 143, 144]. 
More detailed information on steel protection in $\mathrm{HCl}$ and $\mathrm{H}_{2} \mathrm{SO}_{4}$ solutions $\left(t=0-200^{\circ} \mathrm{C}\right)$ by composite triazole-based CIs is summarized in review [10].

The next group of composite NFHC-containing CIs are binary mixtures of sulfurcontaining molecular (thiourea, phenylthiourea, $N$-tolylthiourea, diphenylthiourea, Captax) and anionic (sodium diethyldithiocarbamate (SDEDTC), KNCS) additives. They enhance the protective effect of substituted triazoles on steel corrosion in $\mathrm{H}_{2} \mathrm{SO}_{4}$ solutions $[125,134,135,223]$. For example, the addition of $10 \mathrm{mM} N$-cinnamylidene- $1 H-1,2,4-$ triazole-3-amine (CTA) inhibits corrosion of steel 3 in $2 \mathrm{M} \mathrm{H}_{2} \mathrm{SO}_{4}\left(60^{\circ} \mathrm{C}\right)$ only 2.2-fold, while the $5 \mathrm{mM}$ CTA $+5 \mathrm{mM}$ SDEDTC and $5 \mathrm{mM} \mathrm{CTA}+5 \mathrm{mM}$ KNCS formulations provide 83 - and 65-fold inhibition. Individual $10 \mathrm{mM}$ SDEDTC and $10 \mathrm{mM}$ KNCS decrease the corrosion rate only 1.1- and 2.6-fold under similar conditions [125]. In solutions of phosphoric, formic, acetic, and citric acids, substituted triazoles weakly inhibit the corrosion of low-carbon steels. However, a substituted triazole, IFKhAN-92, and sulfur-containing additives use simultaneously allow one to protect steels in these media efficiently. Of particular interest is the formulation of IFKhAN-92 and KNCS that protects low-carbon steels in $\mathrm{H}_{3} \mathrm{PO}_{4}$ solutions [224] and citric acid [225] at temperatures up to $100^{\circ} \mathrm{C}$. Under similar conditions, the IFKhAN-92+SDEDTC formulation inhibits low-carbon steel corrosion in formic and acetic acid solutions [226].

The use of $\mathrm{HCl}$ and $\mathrm{H}_{2} \mathrm{SO}_{4}$ solutions leads to accumulation of $\mathrm{Fe}(\mathrm{III})$ salts in these systems due to dissolution of rust or thermal scale in acids. Steel protection in solutions of such acids containing Fe(III) salts poses a serious problem. In these media, CIs inhibit the reduction of protons on steels, but fail to inhibit the reduction of such a strong oxidant as $\mathrm{Fe}(\mathrm{III})$, which renders the inhibitory protection of the metal unsatisfactory [227]. A unique property of the formulation of IFKhAN-92+KNCS is its ability to slow down the corrosion of low-carbon steel in $\mathrm{H}_{3} \mathrm{PO}_{4}$ solutions containing $\mathrm{FePO}_{4}$ [228]. It turns out that efficient steel protection $\left(t=20-80^{\circ} \mathrm{C}\right)$ by the formulation of IFKhAN-92 + KNCS can be provided not only in $\mathrm{H}_{3} \mathrm{PO}_{4}$ solutions but also in $\mathrm{H}_{2} \mathrm{SO}_{4}+\mathrm{H}_{3} \mathrm{PO}_{4}+\mathrm{Fe}(\mathrm{III})$ mixtures containing as little as $40 \% \mathrm{H}_{3} \mathrm{PO}_{4}[229,230]$. A more interesting result is provided by a three-component $\mathrm{CI}$ comprising IFKhAN-92 + KNCS + urotropine that also inhibits steel corrosion in $\mathrm{HCl}+$ $\mathrm{H}_{3} \mathrm{PO}_{4}+\mathrm{Fe}(\mathrm{III})$ mixtures containing $40 \% \mathrm{H}_{3} \mathrm{PO}_{4}$ [230,231]. A similar effect of the formulation of IFKhAN-92 + KNCS + urotropine is observed for high-strength steel 70S2 KhA in $\mathrm{H}_{2} \mathrm{SO}_{4}+\mathrm{H}_{3} \mathrm{PO}_{4}+\mathrm{Fe}$ (III) [232] and in $\mathrm{HCl}+\mathrm{H}_{3} \mathrm{PO}_{4}+\mathrm{Fe}$ (III) [233]. It is known that 70S2KhA steel shows a tendency for hydrogen absorption in acid media. It was shown that the IFKhAN-92-based three-component formulation considerably suppresses hydrogen absorption by $70 \mathrm{~S} 2 \mathrm{KhA}$ steel. These results are very important for practical purposes since they open new ways for the development of inhibited acid solutions with a wide temperature range that are stable against accumulation of $\mathrm{Fe}(\mathrm{III})$ cations and are based on relatively cheap $\mathrm{HCl}$ and $\mathrm{H}_{2} \mathrm{SO}_{4}$.

Thus, the possibility of developing new high-performance CIs was shown for formulations based on triazole derivatives in a wide range of acid media where they can be used. Composite CIs open new prospects for practical application of NFHCs in the fields 
where they were never used before: high-temperature steel CIs in acid media and CIs of steel in acid solutions containing Fe(III) salts.

\section{Regularities of the mechanism of action of nitrogen-containing five-membered heterocyclic CIs of metals in acid media}

Efficient protection of metals by NFHCs in highly corrosive acid solutions, particularly under high-temperature corrosion conditions or in the presence of corrosion promoters (Fe(III) salts), is possible only if the CIs form polymolecular protective layers on the metal surface strongly bound to the latter. A strong bond between a protective CI layer and a metal surface is provided by chemisorption interaction between a CI monolayer and the metal. The molecules in a CI layer can be bound both physically and chemically. The strongest protective layers are formed by chemically bound CI molecules. There are two ways in which such protective layers are formed: by polymerization of molecules of unsaturated organic compounds $[8,35,36]$ and by formation of polymeric complex compounds from CI molecules and metal cations formed due to corrosion [10]. The second way is possible if the CIs used are NFHCs that are able to form polymeric complexes with cations of $d$-metals [234-239]. Importantly, nitrogen-containing heterocycles in such complex compounds are bidentate ligands. As CIs under consideration manifest the strongest protective effects when used in combinations with other compounds, it is necessary to understand the effect of these substances on the corrosion process.

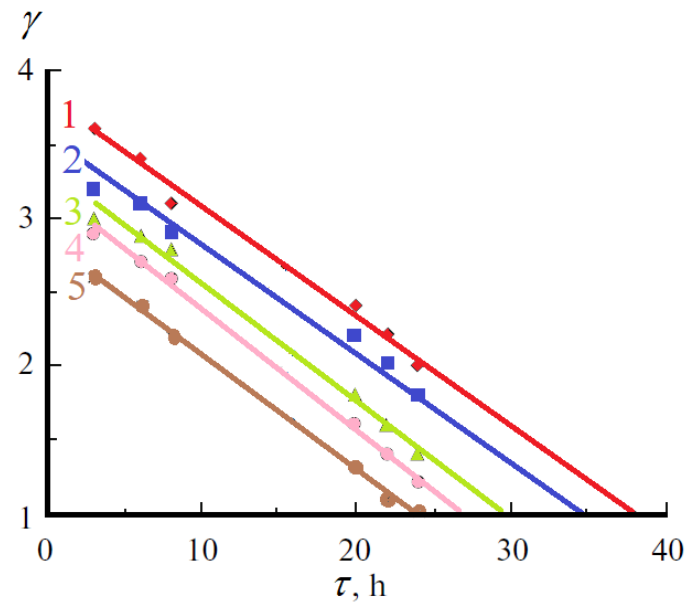

Figure 2. Dependence of coefficient of residual protective effect $(\gamma)$ on time $(\tau)$ at different concentrations of inhibitor, mM: $1-10.0 ; 2-5.0 ; 3-2.5 ; 4-1.25 ; 5-0.625$ [240].

An experimental proof that NFHCs form protective layers on the metal surface in acid solutions is given by the protective aftereffect of these CIs. It was shown [240] that iron samples exposed to $1 \mathrm{M} \mathrm{H}_{2} \mathrm{SO}_{4}$ (room temperature, $24 \mathrm{~h}$ ) inhibited by 1-methyl-3-(3phthalimido-2-hydroxypropyl)-2-iminobenzimidazoline, after washing in distilled water and transferring them to pure $1 \mathrm{M} \mathrm{H}_{2} \mathrm{SO}_{4}$, corroded at a smaller rate than that of samples not conditioned in the inhibited acid (Figure 2). The protective aftereffect of this CI linearly 
decreases with time and lasts from 23 to $38 \mathrm{~h}$. It is the stronger the higher the $C_{\text {in }}$ in the preadsorption solution.

The formation of a polymolecular CI layer on a metal that can provide its efficient protection begins with the formation of a CI monolayer that is chemically bound to the metal surface. The nature of the bond between a CI and a metal can be understood on the basis of its free adsorption energy $\left(-\Delta G_{\text {ads }}\right)$. It is assumed that if $\left(-\Delta G_{\text {ads }}\right)<20 \mathrm{~kJ} / \mathrm{mol}$, its is of physical nature. Only if $\left(-\Delta G_{\text {ads }}\right) \geq 40 \mathrm{~kJ} / \mathrm{mol}$, one can state the high probability that the CI is chemisorbed.

To understand the nature of NFHC interaction with the surfaces of metals in acid solutions, one should systematize the data on their $\left(-\Delta G_{\text {ads }}\right)$. Despite the practically complete absence of any estimates of NFHC adsorption on metals in acid solutions obtained by direct measurements in the available literature, there is a lot of data on the determination of $\left(-\Delta G_{\text {ads }}\right)$ obtained by a less valid method, namely, processing of the results of measurements of metal sample mass losses in inhibited acid solutions. Our analysis of literature data (Table 2 ) shows that the calculated $\left(-\Delta G_{\text {ads }}\right)$ values for some NFHCs reach or exceed the threshold value of $40 \mathrm{~kJ} / \mathrm{mol}$, which allows assuming their chemisorption on metals.

Table 2. Free adsorption energies of nitrogen-containing five-membered organic heterocycles on metals from acid media.

\begin{tabular}{|c|c|c|c|c|}
\hline Inhibitor & $\begin{array}{l}\text { Type of the } \\
\text { metal }\end{array}$ & $\begin{array}{l}\text { Type of the } \\
\text { corrosive } \\
\text { medium }\end{array}$ & $\begin{array}{c}\text { Standard } \\
\text { adsorption free } \\
\text { energy }\left(-\Delta G_{\mathrm{ads}}\right)^{*}, \\
\mathbf{k J} / \mathbf{m o l}\end{array}$ & Ref. \\
\hline \multicolumn{5}{|c|}{ Pyrroles } \\
\hline 2.5-Difuryl-N-methylpyrrole & Mild steel & $\begin{array}{l}1 \mathrm{M} \mathrm{HCl} \\
\left(35^{\circ} \mathrm{C}\right)\end{array}$ & 40.8 & {$[24]$} \\
\hline $\begin{array}{l}\text { 1-(4-Methylphenyl)-1H-pyrrole-2,5- } \\
\text { dione; 1-phenyl-1H-pyrrole-2,5-dione }\end{array}$ & Carbon steel & $\begin{array}{l}1 \mathrm{M} \mathrm{HCl} \\
(\operatorname{room} t)\end{array}$ & $43.9 ; 45.2$ & [25] \\
\hline $\begin{array}{l}\text { 2-Amino-3-methyl-3-phenyl-5- } \\
\text { (phenylthio)-3H-pyrrole-4-carbonitrile; } \\
\text { 2-amino-3-(4-hydroxyphenyl)-3- } \\
\text { methyl-5-(phenylthio)-3H-pyrrole-4- } \\
\text { carbonitrile; } 2 \text {-amino-3-(2,4- } \\
\text { dihydroxyphenyl)-3-methyl-5- } \\
\text { (phenylthio)-3H-pyrrole-4-carbonitrile }\end{array}$ & Mild steel & $\begin{array}{c}1 \mathrm{M} \mathrm{HCl} \\
\left(35-65^{\circ} \mathrm{C}\right)\end{array}$ & $\begin{array}{l}33.8-35.7 \\
34.4-37.3 \\
35.4-39.6\end{array}$ & [28] \\
\hline $\begin{array}{c}\text { 2-Amino-3-methyl-3-(4-nitrophenyl)- } \\
\text { 5-(phenylthio)-3H-pyrrole-4- } \\
\text { carbonitrile }\end{array}$ & Mild steel & $\begin{array}{c}1 \mathrm{M} \mathrm{HCl} \\
\left(35-65^{\circ} \mathrm{C}\right)\end{array}$ & $34.3-39.5$ & {$[29]$} \\
\hline
\end{tabular}




\begin{tabular}{|c|c|c|c|c|}
\hline Inhibitor & $\begin{array}{l}\text { Type of the } \\
\text { metal }\end{array}$ & $\begin{array}{l}\text { Type of the } \\
\text { corrosive } \\
\text { medium }\end{array}$ & $\begin{array}{c}\text { Standard } \\
\text { adsorption free } \\
\text { energy }\left(-\Delta G_{\text {ads }}\right)^{*} \\
\mathrm{~kJ} / \mathbf{m o l}\end{array}$ & Ref. \\
\hline \multicolumn{5}{|c|}{ Pyrrolidines } \\
\hline $\begin{array}{l}\text { Diethyl-2-(2-oxopyrrolidin-1- } \\
\text { yl)ethylphosphonate }\end{array}$ & Mild steel & $\begin{array}{l}0.5 \mathrm{M} \mathrm{H}_{2} \mathrm{SO}_{4} \\
\left(25^{\circ} \mathrm{C}\right)\end{array}$ & 29.9 & [32] \\
\hline $\begin{array}{l}\text { 1-((Pyridin-2-ylamino)(pyridin-4- } \\
\text { yl)methyl)pyrrolidine-2,5-dione }\end{array}$ & Mild steel & $\begin{array}{c}1 \mathrm{M} \mathrm{HCl} \\
\left(32-62^{\circ} \mathrm{C}\right)\end{array}$ & $32.9-36.1$ & [33] \\
\hline $\begin{array}{c}N, N \text {-Dipropargylpyrrolidium bromide; } \\
N \text {-dodecyl- } N \text {-propargylpyrrolidium } \\
\text { bromide; } N \text {-hexadecyl- } N \text { - } \\
\text { propargylpyrrolidium bromide }\end{array}$ & Mild steel & $\begin{array}{c}1 \mathrm{M} \mathrm{HCl} \\
\left(25^{\circ} \mathrm{C}\right)\end{array}$ & $39.4 ; 42.1 ; 43.6$ & [34] \\
\hline Sodium pyrrolidine dithiocarboxylate & $\begin{array}{c}\text { Monel } \\
\text { copper alloy }\end{array}$ & $\begin{array}{l}5 \% \mathrm{HCl} \\
(\operatorname{room} t)\end{array}$ & 31 & [39] \\
\hline Polyvinyl pyrollidone & Mild steel & $\begin{array}{l}1 \mathrm{M} \mathrm{H}_{2} \mathrm{SO}_{4} \\
\left(30-60^{\circ} \mathrm{C}\right)\end{array}$ & $\begin{array}{l}\text { 11.2-13.1 } \\
\text { (Temkin } \\
\text { adsorption } \\
\text { isotherm) } \\
10.3-16.4 \\
\text { (El-Awady } \\
\text { adsorption } \\
\text { isotherm) }\end{array}$ & [40] \\
\hline Polyvinyl pyrrolidone & Carbon steel & $\begin{array}{c}1 \mathrm{M} \mathrm{HCl} \\
\left(30^{\circ} \mathrm{C}\right)\end{array}$ & 31.6 & [41] \\
\hline $\begin{array}{l}\text { Co-poly(maleic anhydride-N-vinyl-2- } \\
\text { pyrrolidone) }\end{array}$ & Mild steel & $\begin{array}{l}2 \mathrm{M} \mathrm{H}_{2} \mathrm{SO}_{4} \\
\left(25-50^{\circ} \mathrm{C}\right)\end{array}$ & $30.7-31.7$ & [42] \\
\hline Polyvinyl pyrollidone & Mild steel & $\begin{array}{l}0.5 \mathrm{M} \mathrm{H}_{2} \mathrm{SO}_{4} \\
\quad\left(30-60^{\circ} \mathrm{C}\right)\end{array}$ & $\begin{array}{l}4.5-12.3 \\
\text { (Langmuir } \\
\text { adsorption } \\
\text { isotherm) } \\
\text { 17.4-18.6 } \\
\text { (Temkin } \\
\text { adsorption } \\
\text { isotherm) }\end{array}$ & [43] \\
\hline Poly-(vinylpyrrolidone-methylaniline) & Mild steel & $\begin{array}{c}1 \mathrm{M} \mathrm{HCl} \\
\left(30-70^{\circ} \mathrm{C}\right)\end{array}$ & $\begin{array}{c}16.7-22.2 \\
\text { (El-Awady } \\
\text { adsorption } \\
\text { isotherm) }\end{array}$ & [44] \\
\hline
\end{tabular}




\begin{tabular}{|c|c|c|c|c|}
\hline Inhibitor & $\begin{array}{l}\text { Type of the } \\
\text { metal }\end{array}$ & $\begin{array}{l}\text { Type of the } \\
\text { corrosive } \\
\text { medium }\end{array}$ & $\begin{array}{c}\text { Standard } \\
\text { adsorption free } \\
\text { energy }\left(-\Delta G_{\mathrm{ads}}\right)^{*} \\
\mathrm{~kJ} / \mathbf{m o l}\end{array}$ & Ref. \\
\hline \multicolumn{5}{|c|}{ Imidazoles } \\
\hline $\begin{array}{l}\text { 2-Ethyl-4-methylimidazole; } 1 \text { - } \\
\text { benzylimidazole }\end{array}$ & $\begin{array}{l}\text { API X65 } \\
\text { steel }\end{array}$ & $\begin{array}{l}1 \mathrm{M} \mathrm{HCl} \\
(\operatorname{room} t)\end{array}$ & 26.1 & [49] \\
\hline $\begin{array}{c}\text { 2-(1,4,5-Triphenyl- } 1 H \text {-imidazol-2-yl) } \\
\text { phenol; 3-methoxy-4-(1,4,5-triphenyl- } \\
\text { 1H-imidazol-2-yl)phenol }\end{array}$ & Mild steel & $\begin{array}{l}1 \mathrm{M} \mathrm{HCl} \\
\left(25^{\circ} \mathrm{C}\right)\end{array}$ & $45.8 ; 45.4$ & {$[52]$} \\
\hline $\begin{array}{l}\text { 2-(4-Chlorophenyl)-1,4,5-triphenyl- } \\
\text { 1H-imidazole; } 1,4,5 \text {-triphenyl-2- }(p \text { - } \\
\text { tolyl)-1H-imidazole }\end{array}$ & Mild steel & $\begin{array}{l}0.5 \mathrm{M} \mathrm{H}_{2} \mathrm{SO}_{4} \\
\left(25^{\circ} \mathrm{C}\right)\end{array}$ & $42.1 ; 41.7$ & {$[53]$} \\
\hline $\begin{array}{l}\text { Imidazolium Gemini surfactant }\left[\mathrm{C}_{14-4-}\right. \\
\left.\mathrm{C}_{14 \mathrm{im}}\right] \mathrm{Br}_{2} ; \text { imidazolium surfactant } \\
{\left[\mathrm{C}_{14 \mathrm{mim}}\right] \mathrm{Br}}\end{array}$ & $\begin{array}{l}\text { A3 carbon } \\
\text { steel }\end{array}$ & $\begin{array}{c}\mathrm{HCl} \\
\left(25-55^{\circ} \mathrm{C}\right)\end{array}$ & $\begin{array}{l}47.2-58.4 \\
41.3-44.0\end{array}$ & [54] \\
\hline $\begin{array}{l}\text { 2-(3-(Carboxymethyl)-1H-imidazol-3- } \\
\text { ium-1-yl)acetate; } 2-(3-(1- \\
\text { carboxyethyl)-1H-imidazol-3-ium-1- } \\
\text { yl)propanoate; } 2 \text {-(3-(1-carboxy-2- } \\
\text { phenylethyl)-1H-imidazol-3-ium-1-yl)- } \\
\text { 3-phenylpropanoate }\end{array}$ & Mild steel & $\begin{array}{l}1 \mathrm{M} \mathrm{HCl} \\
\left(35^{\circ} \mathrm{C}\right)\end{array}$ & $37.0 ; 37.5 ; 37.6$ & {$[55]$} \\
\hline $\begin{array}{l}\text { 2(-3(-1-Caboxy-2-hydroxyethyl)- } 1 H \text { - } \\
\text { imidazol-3-ium-1-yl)-3- } \\
\text { hydroxypropanoate; } 2-(3-(1-\text { caboxy- } \\
\text { 3methylthio)propyl)-1H-imidazol-3- } \\
\text { ium-1-yl)-4-mercaptobutanoate }\end{array}$ & Mild steel & $\begin{array}{l}1 \mathrm{M} \mathrm{HCl} \\
\left(30^{\circ} \mathrm{C}\right)\end{array}$ & $37.6 ; 41.4$ & [57] \\
\hline 1,3-Dioctadecylimidazolium bromide & Mild steel & $\begin{array}{l}1 \mathrm{M} \mathrm{H}_{2} \mathrm{SO}_{4} \\
\quad(\operatorname{room} t)\end{array}$ & 37.9 & {$[58]$} \\
\hline $\begin{array}{c}\text { 1-Ethyl-3-methylimidazolium } \\
\text { tetrafluoroborate; } 1 \text {-butyl-2,3- } \\
\text { dimethylimidazolium tetrafluoroborate; } \\
\text { 1-decyl-3-methylimidazolium } \\
\text { tetrafluoroborate }\end{array}$ & Mild steel & $1 \mathrm{M} \mathrm{HCl}$ & $33.7 ; 34.2 ; 36.6$ & [59] \\
\hline $\begin{array}{l}\text { 1,4-Di[1-methylene-3- } \\
\text { methylimidazolium bromide]benzene }\end{array}$ & Mild steel & $\begin{array}{l}1 \mathrm{M} \mathrm{H}_{2} \mathrm{SO}_{4} \\
\quad\left(22^{\circ} \mathrm{C}\right)\end{array}$ & 17.5 & {$[60]$} \\
\hline $\begin{array}{l}\text { 1-Vinyl-3-aminopropylimidazolium } \\
\text { hexafluorophosphate; 1-vinyl-3- } \\
\text { aminopropylimidazolium } \\
\text { tetrafluoroborate }\end{array}$ & Carbon steel & $\begin{array}{c}1 \mathrm{M} \mathrm{HCl} \\
\left(25-45^{\circ} \mathrm{C}\right)\end{array}$ & $\begin{array}{l}33.0-34.9 \\
29.9-30.4\end{array}$ & {$[61]$} \\
\hline
\end{tabular}




\begin{tabular}{|c|c|c|c|c|}
\hline Inhibitor & $\begin{array}{l}\text { Type of the } \\
\text { metal }\end{array}$ & $\begin{array}{l}\text { Type of the } \\
\text { corrosive } \\
\text { medium }\end{array}$ & $\begin{array}{c}\text { Standard } \\
\text { adsorption free } \\
\text { energy }\left(-\Delta G_{\text {ads }}\right) * \\
\mathbf{k J} / \mathbf{m o l}\end{array}$ & Ref. \\
\hline $\begin{array}{l}\text { 1-Vinyl-3-methylimidazolium iodide; } \\
\text { 1-vinyl-3-proplylimidazolium iodide; } \\
\text { 1-vinyl-3-butylimidazolium iodide }\end{array}$ & X70 steel & $\begin{array}{l}0.5 \mathrm{M} \mathrm{H}_{2} \mathrm{SO}_{4} \\
\left(25-45^{\circ} \mathrm{C}\right)\end{array}$ & $\begin{array}{l}30.9-31.0 \\
31.3-31.4 \\
31.1-31.9\end{array}$ & [63] \\
\hline $\begin{array}{l}\text { 1-Butyl-3-methylimidazolium chloride; } \\
\text { 1-butyl-3-methylimidazolium } \\
\text { acetate; 1-butyl-3-methylimidazolium } \\
\text { trifluoromethanesulphonate }\end{array}$ & Mild steel & $1 \mathrm{M} \mathrm{HCl}$ & $\begin{array}{l}33.1-34.9 \\
32.9-36.2 \\
34.0-37.0\end{array}$ & [64] \\
\hline $\begin{array}{c}\text { Poly[3-butyl-1-vinylimidazolium } \\
\text { bromide }\end{array}$ & Mild steel & $\begin{array}{l}1 \mathrm{M} \mathrm{HCl} \\
\left(25^{\circ} \mathrm{C}\right)\end{array}$ & $\begin{array}{l}25.1 \text { (Frumkin } \\
\text { adsorption } \\
\text { isotherm) }\end{array}$ & {$[65]$} \\
\hline $\begin{array}{l}\text { 3-Hexadecyl-1-methyl-1H-imidazol-3- } \\
\text { ium bromide; } 3 \text {-hexadecyl-1,2- } \\
\text { dimethyl- } 1 H \text {-imidazol-3-ium bromide }\end{array}$ & Mild steel & $\begin{array}{c}1 \mathrm{M} \mathrm{HCl} \\
\left(25-55^{\circ} \mathrm{C}\right)\end{array}$ & $\begin{array}{l}17.0-19.1 \\
17.6-18.7\end{array}$ & {$[66]$} \\
\hline 1-Decyl-3-methylimidazolium chloride & P110 steel & $\begin{array}{l}15 \% \mathrm{HCl} \\
\left(35^{\circ} \mathrm{C}\right)\end{array}$ & $\begin{array}{l}24.1 \text { (El-Awady } \\
\text { adsorption } \\
\text { isotherm) }\end{array}$ & [67] \\
\hline 4-Phenylimidazole & $\begin{array}{l}\text { Copper } \\
(99.99 \% \\
\text { purity })\end{array}$ & $\begin{array}{l}0.5 \mathrm{M} \mathrm{H}_{2} \mathrm{SO}_{4} \\
\quad\left(25^{\circ} \mathrm{C}\right)\end{array}$ & 29.2 & {$[68]$} \\
\hline $\begin{array}{l}\text { 1-Allyl-3-ethylimidazolium bromide; } \\
\text { 1-Allyl-3-butylimidazolium bromide; } \\
\text { 1-Allyl-3-hexylimidazolium bromide; } \\
\text { 1-Allyl-3-octylimidazolium bromide }\end{array}$ & $\begin{array}{l}\text { Copper } \\
(99.8 \% \\
\text { purity })\end{array}$ & $\begin{array}{l}0.5 \mathrm{M} \mathrm{H}_{2} \mathrm{SO}_{4} \\
\left(25^{\circ} \mathrm{C}\right)\end{array}$ & $36-41$ & [69] \\
\hline Imidazole; methyl imidazole & $\begin{array}{l}\text { Aluminum } \\
\text { (99.99\% } \\
\text { purity) }\end{array}$ & $\begin{array}{l}0.5 \mathrm{M} \mathrm{HCl} \\
\quad\left(30^{\circ} \mathrm{C}\right)\end{array}$ & $\begin{array}{l}26.6 ; 29.5 \\
\text { (Frumkin } \\
\text { adsorption } \\
\text { isotherm) }\end{array}$ & {$[72]$} \\
\hline \multicolumn{5}{|c|}{ Imidazolines } \\
\hline $\begin{array}{l}N \text {-(2-(2-tridecyl-4,5-dihydro-1H- } \\
\text { imidazol-1-yl)ethyl)tetradecanamide }\end{array}$ & $\begin{array}{c}\text { St37 steel } \\
\text { (procured } \\
\text { from } \\
\text { Erdemir } \\
\text { Steel Co., } \\
\text { Turkey) }\end{array}$ & $\begin{array}{l}15 \% \mathrm{HCl} \\
\left(25^{\circ} \mathrm{C}\right)\end{array}$ & 27.1 & [75] \\
\hline $\begin{array}{l}\text { 2-Methyl-4-phenyl-1-tosyl-4,5- } \\
\text { dihydro- } 1 H \text {-imidazole }\end{array}$ & $\begin{array}{l}\text { P110 carbon } \\
\text { steel }\end{array}$ & $\begin{array}{l}1 \mathrm{M} \mathrm{HCl} \\
\left(60^{\circ} \mathrm{C}\right)\end{array}$ & 28.3 & {$[76]$} \\
\hline
\end{tabular}




\begin{tabular}{|c|c|c|c|c|}
\hline Inhibitor & $\begin{array}{l}\text { Type of the } \\
\text { metal }\end{array}$ & $\begin{array}{l}\text { Type of the } \\
\text { corrosive } \\
\text { medium }\end{array}$ & $\begin{array}{c}\text { Standard } \\
\text { adsorption free } \\
\text { energy }\left(-\Delta G_{\text {ads }}\right)^{*} \\
\mathbf{k J} / \mathbf{m o l}\end{array}$ & Ref. \\
\hline $\begin{array}{l}\text { 2-(2-Tribromomethyl-4,5-dihydro- } \\
\text { imidazol-1-yl)-ethylamine }\end{array}$ & Mild steel & $\begin{array}{c}1 \mathrm{M} \mathrm{HCl} \\
\left(30^{\circ} \mathrm{C}\right)\end{array}$ & 38.9 & [78] \\
\hline $\begin{array}{l}\text { 2-(2-Trifluoromethyl-4,5-dihydro- } \\
\text { imidazol-1-yl)-ethylamine; 2-(2- } \\
\text { trichloromethyl-4,5-dihydro-imidazol- } \\
\text { 1-yl)-ethylamine }\end{array}$ & Mild steel & $\begin{array}{c}1 \mathrm{M} \mathrm{HCl} \\
\left(30^{\circ} \mathrm{C}\right)\end{array}$ & $34.7 ; 39.7$ & [79] \\
\hline \multicolumn{5}{|c|}{ Imidazolidines } \\
\hline $\begin{array}{l}\text { 3-Methyl-5,5'-diphenylimidazolidine- } \\
\text { 2,4-dione }\end{array}$ & Mild steel & $\begin{array}{c}1 \mathrm{M} \mathrm{HCl} \\
\left(25^{\circ} \mathrm{C}\right)\end{array}$ & 40.3 & [80] \\
\hline \multicolumn{5}{|c|}{ Pyrazoles } \\
\hline $\begin{array}{l}\text { 2-(Bis }((1 H \text {-pyrazol-1- } \\
\text { yl)methyl)amino)pyrimidine-4,6-diol }\end{array}$ & Mild steel & $\begin{array}{l}1 \mathrm{M} \mathrm{HCl} \\
\left(25^{\circ} \mathrm{C}\right)\end{array}$ & 40.2 & [82] \\
\hline $\begin{array}{l}\text { 1,5-Dimethyl- } 1 H \text {-pyrazole-3- } \\
\text { carbohydrazide }\end{array}$ & Mild steel & $\begin{array}{c}1 \mathrm{M} \mathrm{HCl} \\
\left(35^{\circ} \mathrm{C}\right)\end{array}$ & 41.8 & [84] \\
\hline $\begin{array}{l}\text { N1,N1-Bis(2-(bis }((3,5-d i m e t h y l-1 H- \\
\text { pyrazol-1-yl) methyl) amino)ethyl)- } \\
\text { N2,N2-bis((3,5-dimethyl-1H-pyrazol- } \\
\text { 1-yl) methyl)ethane-1,2-diamine; } \\
\text { diethyl 1,1'-(((4-acetylphenyl) } \\
\text { azanediyl)-bis(methylene))-bis(5- } \\
\text { methyl-1H-pyrazole-3-carboxylate) }\end{array}$ & Mild steel & $\begin{array}{l}1 \mathrm{M} \mathrm{HCl} \\
\left(35^{\circ} \mathrm{C}\right)\end{array}$ & 41.8 & {$[85]$} \\
\hline $\begin{array}{c}(E) \text {-N'-Benzylidene-2-(3,5-dimethyl- } \\
\text { 1H-pyrazol-1-yl)acetohydrazide; }(E)- \\
\text { N'-(4-chlorobenzylidene)-2-(3,5- } \\
\text { dimethyl-1H-pyrazol-1- } \\
\text { yl)acetohydrazide }\end{array}$ & Mild steel & $\begin{array}{l}1 \mathrm{M} \mathrm{HCl} \\
\left(35^{\circ} \mathrm{C}\right)\end{array}$ & $40.2 ; 43.2$ & [86] \\
\hline $\begin{array}{c}\text { (E)-5-(4-(Dimethylamino)phenyl)-3-(4- } \\
\text { (dimethylamino)styryl)-2,3-dihydro- } \\
\text { 1H-pyrazole-1-carbothioamide }\end{array}$ & Mild steel & $\begin{array}{c}1 \mathrm{M} \mathrm{HCl} \\
\left(30-60^{\circ} \mathrm{C}\right)\end{array}$ & $43.0-44.7$ & [87] \\
\hline 2-(3-Methyl-1H-pyrazol-5-yl)pyridine & Mild steel & $\begin{array}{l}1 \mathrm{M} \mathrm{HCl} \\
\left(35^{\circ} \mathrm{C}\right)\end{array}$ & $\begin{array}{l}42 \text { (Frumkin } \\
\text { adsorption } \\
\text { isotherm) }\end{array}$ & [88] \\
\hline
\end{tabular}




\begin{tabular}{|c|c|c|c|c|}
\hline Inhibitor & $\begin{array}{c}\text { Type of the } \\
\text { metal }\end{array}$ & $\begin{array}{l}\text { Type of the } \\
\text { corrosive } \\
\text { medium }\end{array}$ & $\begin{array}{c}\text { Standard } \\
\text { adsorption free } \\
\text { energy }\left(-\Delta G_{\text {ads }}\right)^{*} \\
\mathrm{~kJ} / \mathbf{m o l}\end{array}$ & Ref. \\
\hline $\begin{array}{l}\text { (E)-5-Amino-3-(4-methoxyphenyl)- } N^{\prime}- \\
\text { (1-(4-methoxyphenyl)ethylidene)-1H- } \\
\text { pyrazole-4-carbohydrazide; }(E)-5- \\
\text { amino- } N^{\prime} \text {-(4-chlorobenzylidene)-3-(4- } \\
\text { chlorophenyl)-1H-pyrazole-4- } \\
\text { carbohydrazide }\end{array}$ & Mild steel & $\begin{array}{c}15 \% \mathrm{HCl} \\
\left(30-60^{\circ} \mathrm{C}\right)\end{array}$ & $\begin{array}{l}36.8-39.9 \\
36.5-39.3\end{array}$ & [89] \\
\hline $\begin{array}{l}\text { 1-\{[Benzyl-(2-cyano-ethyl)-amino]- } \\
\text { methyl }\}-5 \text {-methyl-1H-pyrazole-3- } \\
\text { carboxylic acid ethyl ester }\end{array}$ & Mild steel & $\begin{array}{c}1 \mathrm{M} \mathrm{HCl} \\
\left(35-70^{\circ} \mathrm{C}\right)\end{array}$ & $37.1-42.0$ & {$[90]$} \\
\hline $\begin{array}{l}N 1, N 1, N 3, N 3 \text {-Tetrakis }((3,5 \text {-dimethyl- } \\
\text { 1H-pyrazol-1-yl)methyl)propane-1,3- } \\
\text { diamine; } N 1, N 1, N 2, N 2 \text {-tetrakis- }((3,5- \\
\text { dimethyl-1H-pyrazol-1- } \\
\text { yl)methyl)benzene-1,2-diamine }\end{array}$ & Mild steel & $\begin{array}{c}1 \mathrm{M} \mathrm{HCl} \\
\left(40-70^{\circ} \mathrm{C}\right)\end{array}$ & $\begin{array}{l}38.4-39.3 \\
39.3-40.1\end{array}$ & [91] \\
\hline $\begin{array}{c}3^{\prime} \text {-(3,4-Dichlorophenyl)-2',3',6,7,8,9- } \\
\text { hexahydro-2'-phenyl-5'- } \\
\text { styrylspiro[benzocyclo-heptene- } \\
\text { 6(5H),4'-(4H-pyrazol)]-5-one }\end{array}$ & Copper & $\begin{array}{l}2 \mathrm{M} \mathrm{HNO}_{3} \\
\left(25-50^{\circ} \mathrm{C}\right)\end{array}$ & $\begin{array}{l}10.3-11.4 \\
\text { (Freundlich } \\
\text { adsorption } \\
\text { isotherm) }\end{array}$ & [95] \\
\hline \multicolumn{5}{|c|}{ Pyrazolines } \\
\hline $\begin{array}{c}\text { [5-Hydroxy-3-phenyl-5- } \\
\text { (phenylamino)-4,5-dihydro-1H- } \\
\text { pyrazol-1-yl](phenyl)methanone; } \\
\text { (2-hydroxyphenyl)[5-hydroxy-3- } \\
\text { phenyl-5-(phenylamino)-4,5-dihydro- } \\
\text { 1H-pyrazol-1-yl]methanone; } \\
\text { (2-aminophenyl)[5-hydroxy-3-phenyl- } \\
\text { 5-(phenylamino)-4,5-dihydro-1H- } \\
\text { pyrazol-1-yl]methanone; } \\
\text { 5-hydroxy-3-phenyl-5-(phenylamino)- } \\
\text { 4,5-dihydro-1H-pyrazole-1- } \\
\text { carbothioamide }\end{array}$ & Mild steel & $\begin{array}{c}1 \mathrm{M} \mathrm{H}_{2} \mathrm{SO}_{4} \\
\left(30^{\circ} \mathrm{C}\right)\end{array}$ & $\begin{array}{c}26.2 ; 28.6 ; 30.5 ; \\
30.3\end{array}$ & [96] \\
\hline $\begin{array}{l}\text { 2-(4-(5-( } p \text {-Tolyl)-4,5- } \\
\text { dihydro-1H-pyrazol-3- } \\
\text { yl)phenoxy)acetic acid; } \\
\text { 2-(4-(5-(4-nitrophenyl)-4,5- } \\
\text { dihydro-1H-pyrazol-3- } \\
\text { yl)phenoxy)acetic acid }\end{array}$ & Mild steel & $\begin{array}{c}1 \mathrm{M} \mathrm{HCl} \\
\left(30^{\circ} \mathrm{C}\right)\end{array}$ & $36 ; 37$ & [97] \\
\hline
\end{tabular}




\begin{tabular}{|c|c|c|c|c|}
\hline Inhibitor & $\begin{array}{l}\text { Type of the } \\
\text { metal }\end{array}$ & $\begin{array}{l}\text { Type of the } \\
\text { corrosive } \\
\text { medium }\end{array}$ & $\begin{array}{c}\text { Standard } \\
\text { adsorption free } \\
\text { energy }\left(-\Delta G_{\mathrm{ads}}\right)^{*} \\
\mathrm{~kJ} / \mathbf{m o l}\end{array}$ & Ref. \\
\hline $\begin{array}{l}\text { 2-(4-(4,5-Dihydro-3-(4- } \\
\text { methoxyphenyl)-1H-pyrazol-5- } \\
\text { yl)phenoxy)acetic acid; } \\
\text { 2-(4-(4,5-dihydro-3-p-tolyl-1H- } \\
\text { pyrazol-5-yl)phenoxy)acetic acid }\end{array}$ & Mild steel & $\begin{array}{l}1 \mathrm{M} \mathrm{HCl} \\
\left(30^{\circ} \mathrm{C}\right)\end{array}$ & $34.4 ; 33.9$ & {$[98]$} \\
\hline $\begin{array}{l}\text { 5-(4-(Dimethylamino)phenyl)-3- } \\
\text { phenyl-4,5-dihydro-1H-pyrazole-1- } \\
\text { carboxamide; } \\
\text { (E)-5-(4-(dimethylamino)phenyl)-3-(4- } \\
\text { (dimethylamino)styryl)-4,5-dihydro- } \\
\text { 1H-pyrazole-1-carboxamide }\end{array}$ & Mild steel & $\begin{array}{c}1 \mathrm{M} \mathrm{HCl} \\
\left(30-60^{\circ} \mathrm{C}\right)\end{array}$ & $\begin{array}{l}33.8-36.3 \\
34.6-40.0\end{array}$ & [99] \\
\hline $\begin{array}{l}\text { 5,5' -(1,4-Phenylene)-bis-[1-formyl-4,5- } \\
\text { dihydro-3-phenyl-1H-pyrazole }]\end{array}$ & Carbon steel & $\begin{array}{l}1 \mathrm{M} \mathrm{HCl} \\
\left(60^{\circ} \mathrm{C}\right)\end{array}$ & 34.7 & [100] \\
\hline \multicolumn{5}{|c|}{ 1,2,3-Triazoles } \\
\hline $\begin{array}{c}\text { (1-p-Tolyl-1 } H \text {-1,2,3-triazol-4-yl) } \\
\text { methanol }\end{array}$ & Mild steel & $\begin{array}{c}1 \mathrm{M} \mathrm{HCl} \\
\left(25-60^{\circ} \mathrm{C}\right)\end{array}$ & $\begin{array}{l}46.2-50.2 \\
\text { (Temkin } \\
\text { adsorption } \\
\text { isotherm) }\end{array}$ & [103] \\
\hline $\begin{array}{l}\text { (1-(4-Aminophenyl)-1H-1,2,3-triazol- } \\
\text { 4-yl)methanol; } \\
\text { 1-(1-(4-aminophenyl)-5-methyl-1H- } \\
\text { 1,2,3-triazol-4-yl)ethanol }\end{array}$ & Carbon steel & $\begin{array}{l}1 \mathrm{M} \mathrm{HCl} \\
\left(25^{\circ} \mathrm{C}\right)\end{array}$ & $33.6 ; 33.1$ & [104] \\
\hline $\begin{array}{l}\text { (R)-Benzyl-2-amino-3- }((1- \\
\quad((2 R, 3 R, 4 S, 5 R, 6 S)-3,4,5- \\
\text { tris(benzyloxy)-6-methoxytetrahydro- } \\
2 H \text {-pyran-2-yl)methyl)-1H-1,2,3- } \\
\text { triazol-4-yl)methoxy)propanoate; } \\
\quad(2 R) \text {-benzyl-2-amino-3-((1- } \\
\quad(((2 R, 3 R, 4 S, 5 R, 6 S)-3,4,5- \\
\text { tris(benzyloxy)-6-methoxytetrahydro- } \\
\text { 2H-pyran-2-yl)methyl)-1H-1,2,3- } \\
\text { triazol-4-yl)methoxy)butanoate; }\end{array}$ & Mild steel & $\begin{array}{l}1 \mathrm{M} \mathrm{HCl} \\
\left(25^{\circ} \mathrm{C}\right)\end{array}$ & $37.6 ; 38.4 ; 37.7$ & {$[105]$} \\
\hline $\begin{array}{c}\text { (2R)-benzyl-2-amino-3- }((1- \\
(((2 R, 3 R, 4 S, 5 \mathrm{~S}, 6 S)-3,4,5- \\
\text { tris(benzyloxy)-6-methoxytetrahydro- } \\
2 H \text {-pyran-2-yl)methyl)-1H-1,2,3- } \\
\text { triazol-4-yl)methoxy)butanoate }\end{array}$ & & & & \\
\hline
\end{tabular}




\begin{tabular}{|c|c|c|c|c|}
\hline Inhibitor & $\begin{array}{l}\text { Type of the } \\
\text { metal }\end{array}$ & $\begin{array}{l}\text { Type of the } \\
\text { corrosive } \\
\text { medium }\end{array}$ & $\begin{array}{c}\text { Standard } \\
\text { adsorption free } \\
\text { energy }\left(-\Delta G_{\text {ads }}\right)^{*} \\
\mathbf{k J} / \mathbf{m o l}\end{array}$ & Ref. \\
\hline 1-Benzyl-4-phenyl-1H-1,2,3-triazole & Mild steel & $\begin{array}{l}1 \mathrm{M} \mathrm{HCl}(25- \\
\left.55^{\circ} \mathrm{C}\right)\end{array}$ & $35.7-39.8$ & {$[106]$} \\
\hline $\begin{array}{l}\text { (1-Benzyl-1H-1,2,3-triazole-4- } \\
\text { yl)methanol; } \\
\text { (1-(pyridin-4-ylmethyl)-1H-1,2,3- } \\
\text { triazole-4-yl)methanol }\end{array}$ & Mild steel & $\begin{array}{l}1 \mathrm{M} \mathrm{HCl} \\
\left(25^{\circ} \mathrm{C}\right)\end{array}$ & $31.3 ; 36.0$ & [107] \\
\hline $\begin{array}{l}\text { 4-Amino-1-((8-hydroxyquinolin-5- } \\
\text { yl)methyl)-1,2,3-triazole-5-carboxylate }\end{array}$ & Carbon steel & $\begin{array}{l}1 \mathrm{M} \mathrm{HCl} \\
\left(25^{\circ} \mathrm{C}\right)\end{array}$ & 43 & [108] \\
\hline $\begin{array}{l}\text { 1-(5-Methyl-1-phenyl-1H-1,2,3-triazol- } \\
\text { 4-yl)ethanone }\end{array}$ & Mild steel & $\begin{array}{c}1 \mathrm{M} \mathrm{HCl} \\
\left(30^{\circ} \mathrm{C}\right)\end{array}$ & 39.9 & [109] \\
\hline $\begin{array}{c}\text { 4,5-Diethyl-1-[(4-ethyl-2-phenyl-4,5- } \\
\text { dihydro-1,3-oxazol-4-yl)methyl]-4,5- } \\
\text { dihydro-1H-1,2,3-triazole-4,5- } \\
\text { dicarboxylate }\end{array}$ & Mild steel & $\begin{array}{l}1 \mathrm{M} \mathrm{HCl} \\
\left(25^{\circ} \mathrm{C}\right)\end{array}$ & 42.8 & [110] \\
\hline \multicolumn{5}{|c|}{ 1,2,4-Triazoles } \\
\hline $\begin{array}{l}\text { 3,5-Bis }(R) \text {-4-amino-1,2,4-triazole }(R= \\
\text { 4-methoxyphenyl; 2-thienylmethyl) }\end{array}$ & $\begin{array}{l}\text { Mild steel, } \\
\text { carbon steel }\end{array}$ & $\begin{array}{c}1 \mathrm{M} \mathrm{HCl} \\
\left(30^{\circ} \mathrm{C}\right)\end{array}$ & $40.8 ; 45.7$ & $\begin{array}{c}{[111} \\
114]\end{array}$ \\
\hline $\begin{array}{c}\text { 3,5-Bis }(R) \text {-4-amino-1,2,4-triazole } \\
(R=\text { 4-methoxyphenyl; 4-chlorophenyl; } \\
\text { 4-tolyl; 3,4-dimethoxyphenyl })\end{array}$ & Mild steel & $\begin{array}{c}2 \mathrm{M} \mathrm{H}_{3} \mathrm{PO}_{4} \\
\left(35^{\circ} \mathrm{C}\right)\end{array}$ & $\begin{array}{c}41.1 ; 39.8 ; 39.8 ; \\
40.0\end{array}$ & $\begin{array}{c}{[116,} \\
117]\end{array}$ \\
\hline $\begin{array}{l}\text { 4-(4-Hydroxy-3- } \\
\text { methoxybenzyledeneamino)-4H-1,2,4- } \\
\text { triazole-3,5-dimethanol }\end{array}$ & Mild steel & $\begin{array}{l}0.5 \mathrm{M} \mathrm{HCl} \\
\left(30-60^{\circ} \mathrm{C}\right)\end{array}$ & $31.9-36.2$ & [118] \\
\hline $\begin{array}{c}\text { (3-Phenylallylidene) amino-5- } \\
\text { (pyridine-4-yl)-4H-1,2,4-triazole-3- } \\
\text { thiol; } \\
\text { 3-mercapto-5-(pyridine-4-yl)-4H-1,2,4- } \\
\text { triazole-4-yl)imino)methyl)phenol; } \\
\text { (4-nitrobenzylidene) amino)-5- } \\
\text { (pyridine-4-yl)-4H-1,2,4-triazole-3- } \\
\text { thiol }\end{array}$ & Mild steel & $\begin{array}{c}1 \mathrm{M} \mathrm{HCl} \\
\left(35-65^{\circ} \mathrm{C}\right)\end{array}$ & $\begin{array}{l}32.3-37.0 \\
32.0-35.0 \\
31.6-33.4\end{array}$ & [119] \\
\hline
\end{tabular}




\begin{tabular}{|c|c|c|c|c|}
\hline Inhibitor & $\begin{array}{l}\text { Type of the } \\
\text { metal }\end{array}$ & $\begin{array}{l}\text { Type of the } \\
\text { corrosive } \\
\text { medium }\end{array}$ & $\begin{array}{c}\text { Standard } \\
\text { adsorption free } \\
\text { energy }\left(-\Delta G_{\text {ads }}\right)^{*} \\
\mathbf{k J} / \mathbf{m o l}\end{array}$ & Ref. \\
\hline $\begin{array}{c}\text { (3-Bromo-4-fluoro-benzylidene)- } \\
\text { [1,2,4]triazol-4-yl-amine; } \\
\text { (4-trifluoromethyl-benzylidene)- } \\
\text { [1,2,4]triazol-4-yl-amine; } \\
\text { (2-fluoro-4-nitro-benzylidene)- } \\
{[1,2,4] \text { triazol-4-yl-amine }}\end{array}$ & Mild steel & $\begin{array}{l}0.5 \mathrm{M} \mathrm{HCl} \\
\left(30-60^{\circ} \mathrm{C}\right)\end{array}$ & $\begin{array}{l}31.1-34.1 \\
31.0-33.5 \\
30.5-33.0\end{array}$ & [119] \\
\hline $\begin{array}{l}\text { 4-Salicylideneamino-3-phenyl-5- } \\
\text { mercapto-1,2,4-triazole }\end{array}$ & Mild steel & $\begin{array}{c}1 \mathrm{M} \mathrm{HCl} \\
\left(25-70^{\circ} \mathrm{C}\right)\end{array}$ & $32.8-36.1$ & [121] \\
\hline $\begin{array}{l}\text { 5-((Furan-2-yl)methyleneamino)-2H- } \\
\text { 1,2,4-triazole-3-thiol; } \\
\text { 5-((thiophen-2-yl)methyleneamino)- } \\
\text { 2H-1,2,4-triazole-3-thiol }\end{array}$ & Mild steel & $\begin{array}{c}1 \mathrm{M} \mathrm{HCl} \\
\left(27^{\circ} \mathrm{C}\right)\end{array}$ & $34.3 ; 35.6$ & [123] \\
\hline $\begin{array}{l}\text { 3,5-Bis(methylene octadecyl } \\
\text { dimethylammonium chloride)-1,2,4- } \\
\text { triazole }\end{array}$ & Carbon steel & $\begin{array}{l}1 \mathrm{M} \mathrm{HCl} \\
\left(25^{\circ} \mathrm{C}\right)\end{array}$ & 42.1 & {$[128]$} \\
\hline 5-Hexylsulfanyl-1,2,4-triazole & Carbon steel & $\begin{array}{l}1 \mathrm{M} \mathrm{HCl} \\
\left(25^{\circ} \mathrm{C}\right)\end{array}$ & 45.9 & [129] \\
\hline $\begin{array}{l}\text { 5-Octylsulfanyl-1,2,4-triazole; } \\
\text { 5-decylsulfanyl-1,2,4-triazole }\end{array}$ & Mild steel & $\begin{array}{c}1 \mathrm{M} \mathrm{HCl} \\
\left(30^{\circ} \mathrm{C}\right)\end{array}$ & $41.4 ; 42.2$ & [130] \\
\hline $\begin{array}{l}\text { 4- }\{[4-(\text { Dimethylamino)benzylidene }]- \\
\text { amino }\}-5 \text {-methyl- } 4 H-1,2,4 \text {-triazole- } 3- \\
\text { thiol }\end{array}$ & $\begin{array}{l}316 \text { Stainless } \\
\text { steel }\end{array}$ & $\begin{array}{c}2 \mathrm{M} \mathrm{HCl} \\
\left(30-60^{\circ} \mathrm{C}\right)\end{array}$ & $30.9-38.9$ & {$[139]$} \\
\hline $\begin{array}{l}\text { 4-Amino-4H-1,2,4-triazole-3thiol; } \\
\text { 4-amino-5-methyl-4H-1,2,4-triazole- } \\
\text { 3thiol; } \\
\text { 4-amino-5-ethyl-4H-1,2,4-triazole- } \\
\text { 3thiol }\end{array}$ & $\begin{array}{l}\text { Copper } \\
(99.9 \%)\end{array}$ & $\begin{array}{l}0.5 \mathrm{M} \mathrm{HCl} \\
\quad\left(30^{\circ} \mathrm{C}\right)\end{array}$ & $36.5 ; 37.3 ; 37.7$ & {$[145]$} \\
\hline $\begin{array}{l}\text { 3,5-Bis(4-methoxyphenyl)-4-amino- } \\
\text { 1,2,4-triazole }\end{array}$ & $\begin{array}{l}\text { Copper } \\
(99.9 \%)\end{array}$ & $\begin{array}{l}1 \mathrm{M} \mathrm{HCl} \\
\left(30^{\circ} \mathrm{C}\right)\end{array}$ & 42.9 & {$[147]$} \\
\hline \multicolumn{5}{|c|}{ 1,2,3,4-Tetrazoles } \\
\hline $\begin{array}{c}\text { 3,3'-(3,3'-Dimethoxy[1,1'-biphenyl]- } \\
\text { 4,4'-diyl)-bis }(2,5 \text {-diphenyl- } 2 H \text { - } \\
\text { tetrazolium) dichloride }\end{array}$ & $\begin{array}{l}\text { Cold rolled } \\
\text { steel }\end{array}$ & $\begin{array}{l}1 \mathrm{M} \mathrm{HCl} \\
\left(20^{\circ} \mathrm{C}\right)\end{array}$ & 40.7 & {$[151]$} \\
\hline
\end{tabular}




\begin{tabular}{|c|c|c|c|c|}
\hline Inhibitor & $\begin{array}{l}\text { Type of the } \\
\text { metal }\end{array}$ & $\begin{array}{l}\text { Type of the } \\
\text { corrosive } \\
\text { medium }\end{array}$ & $\begin{array}{c}\text { Standard } \\
\text { adsorption free } \\
\text { energy }\left(-\Delta G_{\text {ads }}\right)^{*} \\
\mathbf{k J} / \mathbf{m o l}\end{array}$ & Ref. \\
\hline $\begin{array}{c}\text { (E)-3-Phenyl-2-(1H-tetrazole-5- } \\
\text { yl)acrylonitrile; } \\
\text { (E)-3-(4-nitrophenyl)-2-(1H-tetrazole- } \\
\text { 5-yl)acrylonitrile; } \\
\text { (E)-3-(4-hydroxyphenyl)-2-(1H- } \\
\text { tetrazole-5- yl)acrylonitrile }\end{array}$ & Mild steel & $\begin{array}{c}1 \mathrm{M} \mathrm{HCl} \\
\left(35-65^{\circ} \mathrm{C}\right)\end{array}$ & $\begin{array}{l}34.9-36.7 \\
36.2-38.2 \\
37.6-39.7\end{array}$ & {$[152]$} \\
\hline 2,3,5-Triphenyltetrazolium chloride & $\begin{array}{l}\text { Cold rolled } \\
\text { steel }\end{array}$ & $\begin{array}{c}1 \mathrm{M} \mathrm{H}_{2} \mathrm{SO}_{4} \\
\left(20-50^{\circ} \mathrm{C}\right) \\
7 \mathrm{M} \mathrm{H}_{2} \mathrm{SO}_{4} \\
\left(20^{\circ} \mathrm{C}\right)\end{array}$ & $\begin{array}{l}29.4-32.5 \\
34.5\end{array}$ & $\begin{array}{c}{[155,} \\
159]\end{array}$ \\
\hline $\begin{array}{c}\text { 2,2'-Bis(4-nitrophenyl)-5,5'-diphenyl- } \\
\text { 3,3'-(3,3'-dimethoxy-4,4'- } \\
\text { diphenylene)ditetrazolium chloride }\end{array}$ & $\begin{array}{l}\text { Cold rolled } \\
\text { steel }\end{array}$ & $\begin{array}{l}0.5 \mathrm{M} \mathrm{H}_{2} \mathrm{SO}_{4} \\
\quad\left(25^{\circ} \mathrm{C}\right)\end{array}$ & 41.0 & {$[156]$} \\
\hline $\begin{array}{l}\text { 3-(4,5-Dimethyl-2-thiazolyl)-2,5- } \\
\text { diphenyl-2H-tetrazolium bromide }\end{array}$ & $\begin{array}{l}\text { Cold rolled } \\
\text { steel }\end{array}$ & $\begin{array}{c}1 \mathrm{M} \mathrm{HCl} \\
\left(30^{\circ} \mathrm{C}\right) ; \\
0.5 \mathrm{M} \mathrm{H}_{2} \mathrm{SO}_{4} \\
\left(30^{\circ} \mathrm{C}\right)\end{array}$ & $\begin{array}{l}36.5 \\
33.7\end{array}$ & {$[157]$} \\
\hline $\begin{array}{c}\text { 3,3'-(3,3'-Dimethoxy[1,1'-biphenyl]- } \\
\text { 4,4'-diyl)-bis(2,5-diphenyl- } 2 H \text { - } \\
\text { tetrazolium) dichloride }\end{array}$ & $\begin{array}{l}\text { Cold rolled } \\
\text { steel }\end{array}$ & $\begin{array}{l}1 \mathrm{M} \mathrm{H}_{2} \mathrm{SO}_{4} \\
\quad\left(20^{\circ} \mathrm{C}\right)\end{array}$ & 39.1 & {$[158]$} \\
\hline $\begin{array}{l}\text { 5-Mercapto-1-methyltetrazole; } \\
\text { 1-[2-(dimethylamino)ethyl]-1H- } \\
\text { tetrazole-5-thiol; } \\
\text { 1-(4-hydroxyphenyl)-1H-tetrazole-5- } \\
\text { thiol }\end{array}$ & X80 Steel & $\begin{array}{l}0.5 \mathrm{M} \mathrm{H}_{2} \mathrm{SO}_{4} \\
\quad\left(25^{\circ} \mathrm{C}\right)\end{array}$ & $34.3 ; 33.7 ; 32.7$ & {$[161]$} \\
\hline $\begin{array}{c}\text { 3,3'-(3,3'-Dimethoxy[1,1'-biphenyl]- } \\
\text { 4,4'-diyl)-bis(2,5-diphenyl- } 2 H \text { - } \\
\text { tetrazolium) dichloride; } \\
\text { 2,2'-bis(4-nitrophenyl)-5,5'-diphenyl- } \\
\text { 3,3'-(3,3'-dimethoxy-4,4'- } \\
\text { diphenylene)ditetrazolium chloride; }\end{array}$ & $\begin{array}{l}\text { Cold rolled } \\
\text { steel }\end{array}$ & $\begin{array}{c}2.5 \mathrm{M} \\
\mathrm{H}_{3} \mathrm{CCOOH} \\
\left(20^{\circ} \mathrm{C}\right)\end{array}$ & $37.4 ; 38.7$ & {$[163]$} \\
\hline
\end{tabular}




\begin{tabular}{|c|c|c|c|c|}
\hline Inhibitor & $\begin{array}{l}\text { Type of the } \\
\text { metal }\end{array}$ & $\begin{array}{l}\text { Type of the } \\
\text { corrosive } \\
\text { medium }\end{array}$ & $\begin{array}{c}\text { Standard } \\
\text { adsorption free } \\
\text { energy }\left(-\Delta G_{\mathrm{ads}}\right) * \\
\mathbf{k J} / \mathbf{m o l}\end{array}$ & Ref. \\
\hline $\begin{array}{l}\text { 3-(4,5-Dimethyl-2-thiazolyl)-2,5- } \\
\text { diphenyl-2H-tetrazolium bromide }\end{array}$ & $\begin{array}{l}\text { Cold rolled } \\
\text { steel }\end{array}$ & $\begin{array}{c}1 \mathrm{M} \\
\mathrm{ClCH}_{2} \mathrm{COOH} ; \\
1 \mathrm{M} \\
\mathrm{Cl}_{2} \mathrm{CHCOOH} ; \\
1 \mathrm{M} \\
\mathrm{Cl}_{3} \mathrm{CCOOH} \\
\left(25^{\circ} \mathrm{C}\right)\end{array}$ & $33.1 ; 34.0 ; 34.9$ & {$[164]$} \\
\hline $\begin{array}{l}\text { 5-Phenyltetrazole; } \\
\text { 5-(2-bromophenyl)- } 1 H \text {-tetrazole; } \\
\text { 5-(4-bromophenyl)-2H-tetrazole }\end{array}$ & $\begin{array}{l}\text { Copper } \\
(99.99 \%)\end{array}$ & $\begin{array}{l}0.5 \mathrm{M} \mathrm{H}_{2} \mathrm{SO}_{4} \\
\left(25^{\circ} \mathrm{C}\right)\end{array}$ & $38.2 ; 38.7 ; 37.8$ & [167] \\
\hline $\begin{array}{c}\text { 1-Phenyl- } 1 H \text {-tetrazole-5-thiol; } \\
\text { 1-phenyl-1H-tetrazole; } \\
1 H \text {-tetrazol-5-amine; } \\
1 H \text {-tetrazole }\end{array}$ & Aluminum & $\begin{array}{l}1 \mathrm{M} \mathrm{HCl} \\
\left(25^{\circ} \mathrm{C}\right)\end{array}$ & $\begin{array}{c}32.6 ; 32.1 ; 31.1 ; \\
28.8\end{array}$ & [170] \\
\hline \multicolumn{5}{|c|}{ Complex systems } \\
\hline Indole & $\begin{array}{l}\text { Mild steel } \\
\text { Q235 }\end{array}$ & $\begin{array}{l}0.1 \mathrm{M} \mathrm{H}_{2} \mathrm{SO}_{4} \\
\left(25-45^{\circ} \mathrm{C}\right)\end{array}$ & $28.5-28.8$ & [171] \\
\hline $\begin{array}{l}\text { 5-Aminoindole; } \\
\text { 5-chloroindole; } \\
\text { indole }\end{array}$ & Mild steel & $\begin{array}{l}1 \mathrm{M} \mathrm{H}_{2} \mathrm{SO}_{4} \\
\left(25-55^{\circ} \mathrm{C}\right)\end{array}$ & $\begin{array}{l}30.3-32.0 \\
24.8-28.0 \\
32.7-37.9\end{array}$ & {$[172]$} \\
\hline $\begin{array}{l}N \text {-((1H-Indol-3-yl)(phenyl)methyl)-N- } \\
\text { ethylethanamine; } \\
\text { 3-(phenyl(pyrrolidin-1-yl)methyl)- } 1 H \text { - } \\
\text { indole; } \\
\text { 3-(phenyl(piperidin-1-yl)methyl)- } 1 H \text { - } \\
\text { indole }\end{array}$ & Mild steel & $\begin{array}{c}1 \mathrm{M} \mathrm{HCl} \\
\left(35-65^{\circ} \mathrm{C}\right)\end{array}$ & $\begin{array}{l}33.0-35.5 \\
33.7-36.4 \\
34.2-37.0\end{array}$ & [174] \\
\hline $\begin{array}{l}\text { 4-Hydroxy- } N^{\prime}-[(E)-(1 H \text {-indole-2- } \\
\text { ylmethylidene })] \text { benzohydrazide }\end{array}$ & Mild steel & $\begin{array}{c}1 \mathrm{M} \mathrm{HCl} \\
\left(30-60^{\circ} \mathrm{C}\right)\end{array}$ & $39.8-43.9$ & {$[175]$} \\
\hline Indole-3-acetic acid & Mild steel & $\begin{array}{l}0.5 \mathrm{M} \mathrm{HCl} \\
\left(25^{\circ} \mathrm{C}\right)\end{array}$ & 28.4 & [177] \\
\hline Indole-5-carboxylic acid & Mild steel & $\begin{array}{l}0.5 \mathrm{M} \mathrm{H}_{2} \mathrm{SO}_{4} \\
\left(25-45^{\circ} \mathrm{C}\right)\end{array}$ & $23.8-26.4$ & [178] \\
\hline Indole-3-acetic acid & $\begin{array}{c}\text { Copper } \\
(99.994 \%)\end{array}$ & $\begin{array}{l}0.5 \mathrm{M} \mathrm{H}_{2} \mathrm{SO}_{4} \\
\left(25-55^{\circ} \mathrm{C}\right)\end{array}$ & $25.6-27.5$ & [179] \\
\hline
\end{tabular}




\begin{tabular}{|c|c|c|c|c|}
\hline Inhibitor & $\begin{array}{l}\text { Type of the } \\
\text { metal }\end{array}$ & $\begin{array}{l}\text { Type of the } \\
\text { corrosive } \\
\text { medium }\end{array}$ & $\begin{array}{c}\text { Standard } \\
\text { adsorption free } \\
\text { energy }\left(-\Delta G_{\text {ads }}\right)^{*} \\
\mathrm{~kJ} / \mathbf{m o l}\end{array}$ & Ref. \\
\hline $\begin{array}{l}\text { 3,6-Dibromo-9-phenylcarbazole; } \\
\text { 3-(9H-carbazol-9-yl)-1,2-propanediol }\end{array}$ & Mild steel & $\begin{array}{l}1 \mathrm{M} \mathrm{HCl} \\
\left(30^{\circ} \mathrm{C}\right)\end{array}$ & $\begin{array}{l}23.1 ; 21.9 \\
\text { (Frumkin } \\
\text { adsorption } \\
\text { isotherm) }\end{array}$ & [180] \\
\hline $\begin{array}{c}\text { 6-Methyl-2,3,4,9-tetrahydro-1H- } \\
\text { carbazole; } \\
\text { 9-methyl-9H-carbazole-3-carboxylic } \\
\text { acid }\end{array}$ & Mild steel & $\begin{array}{l}1 \mathrm{M} \mathrm{HCl} \\
(\operatorname{room} t)\end{array}$ & $\begin{array}{l}38.9 ; 37.4 \\
\text { (Frumkin } \\
\text { adsorption } \\
\text { isotherm) }\end{array}$ & [181] \\
\hline $\begin{array}{c}\text { 9H-Pyrido[3,4-b]indole; } \\
\text { 1-methyl-9H-pyrido[3,4-b]indole }\end{array}$ & C38 steel & $\begin{array}{c}1 \mathrm{M} \mathrm{HCl} \\
\left(25^{\circ} \mathrm{C}\right)\end{array}$ & $18 ; 14$ & [182] \\
\hline $\begin{array}{l}\text { BI, 2-Methyl-BI, 5(6)-Carboxy-BI, 2- } \\
\text { Amino-BI, 2-Hydroxymethyl-BI, 2- } \\
\text { Mercapto-BI, 2-Acetonitrile-BI }\end{array}$ & Mild steel & $\begin{array}{l}1 \mathrm{M} \mathrm{HCl} \\
\left(20^{\circ} \mathrm{C}\right)\end{array}$ & $\begin{array}{l}23.2 ; 23.8 ; 28.0 ; \\
30.8 ; 31.0 ; 34.9 ; \\
39.7 \text { (Frumkin } \\
\text { adsorption } \\
\text { isotherm) }\end{array}$ & [186] \\
\hline 2-Mercapto-BI & Mild steel & $\begin{array}{c}1 \mathrm{M} \mathrm{HCl} \\
\left(40-80^{\circ} \mathrm{C}\right)\end{array}$ & $36.6-37.8$ & [187] \\
\hline $\begin{array}{l}\text { 2-(2-Methyl- } 1 H \text {-benzimidazol-1-yl) } \\
\text { acethydrazide; } \\
\text { 2-(2-ethyl- } 1 H \text {-benzimidazol-1-yl) } \\
\text { acethydrazide; } \\
\text { 2-(2-propyl-1H-benzimidazol-1-yl) } \\
\text { acethydrazide }\end{array}$ & Mild steel & $\begin{array}{l}0.5 \mathrm{M} \mathrm{HCl} \\
\left(30-50^{\circ} \mathrm{C}\right)\end{array}$ & $\begin{array}{l}35.5-38.8 \\
37.2-39.9 \\
39.3-41.7\end{array}$ & [188] \\
\hline $\begin{array}{l}\text { 2-(Allylthio)-1H-benzo[d]imidazole; } \\
\text { bis((1H-benzo[d]imidazol-2- } \\
4 \text { yl)thio)methane }\end{array}$ & Mild steel & $\begin{array}{l}1 \mathrm{M} \mathrm{HCl} \\
\left(25^{\circ} \mathrm{C}\right)\end{array}$ & $45.2 ; 46.3$ & [189] \\
\hline $\begin{array}{c}\text { 1,4-Bis(2-(4-chlorophenyl)-1H- } \\
\text { benzo[d]imidazol-1-yl)butane; } \\
\text { 1,4-bis(2-phenyl-1H-benzo[d]imidazol- } \\
\text { 1-yl)butane }\end{array}$ & Mild steel & $\begin{array}{c}1 \mathrm{M} \mathrm{HCl} \\
\left(30^{\circ} \mathrm{C}\right)\end{array}$ & $35.3 ; 33.2$ & {$[190]$} \\
\hline $\begin{array}{c}\text { 2-[(5-Methylpyrazol-3-yl)methyl }] \\
\text { benzimidazole }\end{array}$ & $\begin{array}{l}\text { C38 carbon } \\
\text { steel }\end{array}$ & $\begin{array}{c}1 \mathrm{M} \mathrm{HCl} \\
\left(25^{\circ} \mathrm{C}\right)\end{array}$ & 19.3 & {$[192]$} \\
\hline $\begin{array}{l}\text { 2-Aminomethylbenzimidazole; } \\
\text { bis(2-benzimidazolylmethyl)amine; } \\
\text { tri(2-benzimidazolylmethyl)amine }\end{array}$ & Mild steel & $\begin{array}{l}1 \mathrm{M} \mathrm{HCl} \\
\left(25^{\circ} \mathrm{C}\right)\end{array}$ & $34.3 ; 37.0 ; 38.1$ & [193] \\
\hline
\end{tabular}




\begin{tabular}{|c|c|c|c|c|}
\hline Inhibitor & $\begin{array}{l}\text { Type of the } \\
\text { metal }\end{array}$ & $\begin{array}{l}\text { Type of the } \\
\text { corrosive } \\
\text { medium }\end{array}$ & $\begin{array}{c}\text { Standard } \\
\text { adsorption free } \\
\text { energy }\left(-\Delta G_{\text {ads }}\right)^{*} \\
\mathbf{k J} / \mathbf{m o l}\end{array}$ & Ref. \\
\hline $\begin{array}{l}\text { 1-Butyl-2-(4- } \\
\text { methylphenyl)benzimidazole }\end{array}$ & Mild steel & $\begin{array}{c}1 \mathrm{M} \mathrm{HCl} \\
\left(30^{\circ} \mathrm{C}\right)\end{array}$ & 35.1 & [194] \\
\hline $\begin{array}{l}\text { 2-(1-(Morpholinomethyl)-1H-benzo- } \\
\text { [d]imidazol-2-yl)phenol; } \\
\text { 2-(1-((piperazine-1-yl)methyl)-1H- } \\
\text { benzo[d]imidazol-2-yl)phenol; } \\
\text { 2-(1-((piperidine-1-yl)methyl)- } 1 H \text { - } \\
\text { benzo[d]imidazol-2-yl)phenol }\end{array}$ & N80 steel & $\begin{array}{c}15 \% \mathrm{HCl} \\
\left(30-60^{\circ} \mathrm{C}\right)\end{array}$ & $\begin{array}{l}37.5-40.3 \\
37.3-40.1 \\
36.7-39.4\end{array}$ & [195] \\
\hline 1-Butyl-2-thiobenzylbenzimidazole & Mild steel & $\begin{array}{c}1 \mathrm{M} \mathrm{HCl} \\
\left(30-60^{\circ} \mathrm{C}\right)\end{array}$ & $45.5-46.2$ & [196] \\
\hline $\begin{array}{l}\text { 3-(4-Chlorophenylacylmethyl)-1- } \\
\text { ethylbenzimidazolium tetrafluroborate; } \\
\text { 3-(4-chlorobenzoylmethyl)-1- } \\
\text { methylbenzimidazolium bromide }\end{array}$ & Carbon steel & $\begin{array}{c}1 \mathrm{M} \mathrm{HCl} \\
\left(30-60^{\circ} \mathrm{C}\right)\end{array}$ & $\begin{array}{l}37.7-41.9 \\
32.4-39.5\end{array}$ & $\begin{array}{l}{[197,} \\
198]\end{array}$ \\
\hline $\begin{array}{l}\text { 1-Butyl-3-methyl-1 } H \text {-benzimidazolium } \\
\text { iodide }\end{array}$ & Mild steel & $\begin{array}{c}0.5 \mathrm{M} \mathrm{H}_{2} \mathrm{SO}_{4} \\
\left(25-55^{\circ} \mathrm{C}\right)\end{array}$ & $34.0-34.6$ & [199] \\
\hline $\begin{array}{l}\text { 1,3-Bis[2-(4-methoxyphenyl)-2- } \\
\text { oxoethyl]-1Hbenzimidazol-3-ium } \\
\text { bromide }\end{array}$ & $\begin{array}{l}6061 \mathrm{Al}-15 \\
\text { vol. pct. } \\
\operatorname{SiC}_{(\mathrm{p})} \\
\text { composite } \\
\end{array}$ & $\begin{array}{l}0.1 \mathrm{M} \mathrm{HCl} \text { and } \\
0.1 \mathrm{M} \mathrm{H}_{2} \mathrm{SO}_{4} \\
\left(30-50^{\circ} \mathrm{C}\right)\end{array}$ & $\begin{array}{l}45.4-49.7 \\
37.1-40.8\end{array}$ & [200] \\
\hline $\begin{array}{l}\text { 6-Nitroso-2-phenylimidazo[1,2- } \\
\text { a]pyridine-3 carbaldehyde; } \\
\text { (2-phenylimidazo[1,2-a]pyridin-3- } \\
\text { yl)methanol }\end{array}$ & Carbon steel & $\begin{array}{c}1 \mathrm{M} \mathrm{HCl} \\
\left(25^{\circ} \mathrm{C}\right)\end{array}$ & $46.5 ; 44.1$ & [201] \\
\hline $\begin{array}{l}\text { 4-Amino pyrazolo-pyrimidine; } \\
\text { 3-bromine 4-amino pyrazolo- } \\
\text { pyrimidine; } \\
\text { 3-iodine 4-amino pyrazolo-pyrimidine; } \\
\text { 4-hydroxypyrazolo-pyrimidine; } \\
\text { 4-mercaptopyrazolo-pyrimidine }\end{array}$ & Pure copper & $\begin{array}{c}0.5 \mathrm{M} \mathrm{H}_{2} \mathrm{SO}_{4} \\
\left(25^{\circ} \mathrm{C}\right)\end{array}$ & $\begin{array}{l}32.0 ; 32.4 ; 36.3 \\
\quad 35.9 ; 40.8\end{array}$ & $\begin{array}{l}{[202,} \\
203]\end{array}$ \\
\hline $\begin{array}{c}\text { 1-Dodecyl-5-methyl-1H- } \\
\text { benzo[d][1,2,3]triazol-1-ium bromide }\end{array}$ & Carbon steel & $\begin{array}{l}7 \mathrm{M} \mathrm{H}_{3} \mathrm{PO}_{4} \\
\left(20-80^{\circ} \mathrm{C}\right)\end{array}$ & $37.2-41.6$ & [210] \\
\hline
\end{tabular}

*Adsorption energy data presented without additional clarification were calculated from the Langmuir isotherm.

More valuable and reliable data on NFHC adsorption on metals measured by direct methods are based on a decrease in the capacitance of the double electric layer (DEL) of a 
steel electrode obtained by electrochemical impedance spectroscopy (EIS) and by the ellipsometry method. Studies of adsorption of a substituted triazole, IFKhAN-92, using the EIS method point to the chemisorption character of interaction between the molecules of this $\mathrm{CI}$ and the steel surface. At $t=22^{\circ} \mathrm{C}$, the adsorption of IFKhAN-92 on a cathodically polarized low-carbon steel is described by Temkin's isotherm, while $\left(-\Delta G_{\text {ads }}\right)$ is $42 \mathrm{~kJ} / \mathrm{mol}$ in $2.0 \mathrm{M} \mathrm{HCl}$ [241], $49 \mathrm{~kJ} / \mathrm{mol}$ in $2.0 \mathrm{M} \mathrm{H}_{2} \mathrm{SO}_{4}$ [242], and $51 \mathrm{~kJ} / \mathrm{mol}$ in $2.0 \mathrm{M} \mathrm{H}_{3} \mathrm{PO}_{4}$ [243].

Importantly, the kinetics of IFKhAN-92 adsorption on low-carbon steel from mineral acid solutions is described by the Roginsky-Zeldovich equation characteristic of chemisorption processes. It was stated earlier that IFKhAN-92 is inefficient in steel protection in $2.0 \mathrm{M} \mathrm{H}_{3} \mathrm{PO}_{4}$, but its combination with $0.5 \mathrm{mM} \mathrm{KNCS}$ allows the protection to be enhanced considerably. It was shown that addition of KNCS to $2.0 \mathrm{M} \mathrm{H}_{3} \mathrm{PO}_{4}$ has no effect on $\left(-\Delta G_{\text {ads }}\right)$ of IFKhAN-92 on steel but accelerates its adsorption, as it is clearly demonstrated by the kinetic adsorption isotherms [243]. Apparently, this effect promotes accelerated formation of a protective CI layer that provides the required protection on the metal surface that otherwise quickly degrades in acid solutions. It is worth noting that this is only one of the possible impacts of the addition of KNCS on a corroding system.

EIS data point to the chemisorption interaction between IFKhAN-92 and the surface of chromium-nickel steels. In fact, the $\left(-\Delta G_{\text {ads }}\right)$ values for IFKhAN-92 on cathodically polarized $12 \mathrm{Kh} 18 \mathrm{~N} 10 \mathrm{~T}$ steel calculated according to Temkin's equation are $55 \pm 1$ and $61 \pm 1 \mathrm{~kJ} / \mathrm{mol}$ in $2.0 \mathrm{M} \mathrm{HCl}$ and $2.0 \mathrm{M} \mathrm{H}_{2} \mathrm{SO}_{4}$, accordingly [244]. This result agrees well with the data of ellipsometric studies of its adsorption, according to which the $\left(-\Delta G_{\text {ads }}\right)$ value on this steel in $0.005-0.05 \mathrm{M} \mathrm{HCl}$ and $0.05 \mathrm{M} \mathrm{H}_{2} \mathrm{SO}_{4}$ is $55 \pm 3 \mathrm{~kJ} / \mathrm{mol}$ as calculated by Temkin's isotherm [245].

Important additional information on the nature of the bond between CI molecules and a metal surface can be obtained using quantum-chemical methods. In recent years, more and more studies have been appearing that employ quantum-chemical calculations to explain the inhibitory effect of NFHCs. Detailed analysis of the possibility of using quantum-chemical methods for explanation of the inhibiting effect of organic compounds, including NFHCs, is provided in reviews [246, 247], which allows us to leave this issue beyond the scope of our paper.

Valuable information on the composition and structure of protective layers formed by NFHCs on metal surfaces in acid media is provided by X-ray photoelectron spectroscopy (XPS) combined with layer-by-layer etching of a sample surface by argon ions. This method of XPS measurements allows one to determine the in-depth qualitative and quantitative composition of the protective layer. An important element of such studies is prolonged (up to $18 \mathrm{~min}$ ) ultrasonic washing in distilled water or in acid solutions to remove the CIs from the surface of the metal sample. During this procedure, the CI molecules physically adsorbed on the metal surface are removed from the surface of samples pretreated in an inhibited acid solution. The CI molecules chemically bound to the metal surface are not removed by ultrasonic surface cleaning. Such metal samples demonstrate a protective aftereffect of CIs in 
further corrosion tests in background solutions. The ultrasonic cleaning of the metal surface before XPS measurements provides important information proving the nature of the chemical bond between the CI molecules and the metal, since this method does not allow one to distinguish between CI particles present on the sample surface in the form of a protective layer and those remaining on the metal after incomplete removal of the inhibited corrosive medium.

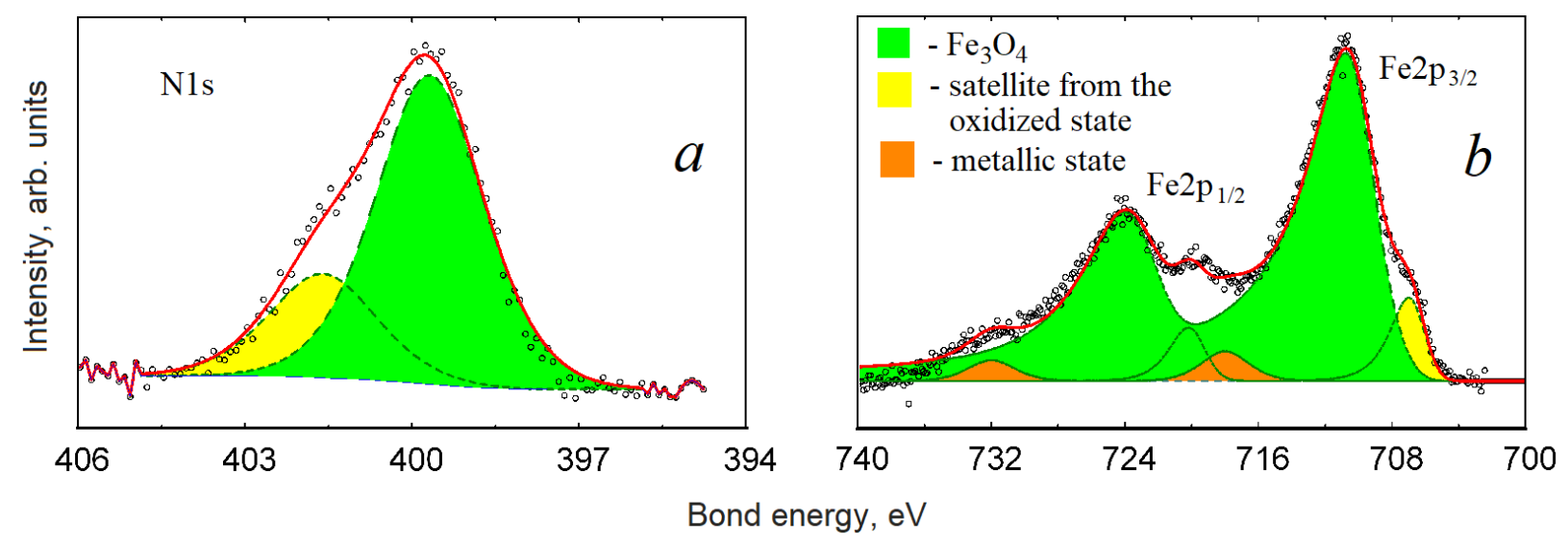

Figure 3. XPS spectra of $\mathrm{N} 1 s(a)$ and $\mathrm{Fe} 2 p(b)$ electrons of surface of steel 3 after holding in $2 \mathrm{M} \mathrm{HCl}+5 \mathrm{mM}$ IFKhAN-92 $\left(24 \mathrm{~h}, 20^{\circ} \mathrm{C}\right)$. With ultrasonic cleaning.

A protective layer with the simplest structure on the surface of steel 3 in $2 \mathrm{M} \mathrm{HCl}\left(22^{\circ} \mathrm{C}\right.$, $24 \mathrm{~h}$ ) is formed by 4-substituted 1,2,4-triazole (IFKhAN-96) [136]. The adsorption of this compound on a steel surface leads to the formation of an apparent CI monolayer that is chemically bound to the metal surface. The organic CI monolayer is located over 4-7 nm thick iron(II) chloride and oxide phases directly adhering to the metal. A 3-substituted 1,2,4triazole (IFKhAN-92) forms a more complex structure on steel 3 in an $\mathrm{HCl}$ solution [248, 249]. According to XPS data, a $4 \mathrm{~nm}$ thick protective layer of an organic CI (no more than 4 monomolecular layers) is formed on steel 3 samples pretreated in $2.0 \mathrm{M} \mathrm{HCl}+$ $5.0 \mathrm{mM}$ IFKhAN-92 $\left(20^{\circ} \mathrm{C}, 24 \mathrm{~h}\right)$. It includes an apparent monolayer of the organic CI no thicker than $2 \mathrm{~nm}$ that is directly chemisorbed on the metal sample surface and the overlying CI molecules physically bound to each other and to the chemisorbed layer. Physically bound $\mathrm{CI}$ layers are removed by sonication in water. The protective monolayer remaining on the metal surface in background $2 \mathrm{M} \mathrm{HCl}\left(20-60^{\circ} \mathrm{C}\right)$ manifests a protective aftereffect $(Z=89-$ $96 \%)$ that is weaker than in the case of a polymolecular CI layer $(Z=95-99 \%)$. It is however sufficiently high to confirm the CI chemisorption on the steel surface (Table 3). The preservation of a CI monolayer on the metal surface after ultrasonic surface cleaning is also confirmed by an XP spectrum of N1s electrons of the steel surface showing different types of nitrogen atoms present in the structure of IFKhAN-92 molecules (Figure 3a). It is necessary to point out that, as shown by the spectra of Fe $2 p$-electrons (Figure 3b), the metal surface under the CI layer is oxidized to iron oxides. A CI layer with a similar structure and similar protective properties is formed in $2.0 \mathrm{M} \mathrm{H}_{2} \mathrm{SO}_{4}+5.0 \mathrm{mM}$ IFKhAN-92 $+5.0 \mathrm{mM}$ 
KNCS (Table 3). However, despite the presence of KNCS in the inhibitory composition, no rhodanide anions are found in the protective layer.

Table 3. Corrosion rates $\left(k, \mathrm{~g} /\left(\mathrm{m}^{2} \cdot \mathrm{h}\right)\right)$ and corrosion inhibition coefficients $(\gamma)$ of steel 3 in $2 \mathrm{M} \mathrm{HCl}, \mathrm{H}_{2} \mathrm{SO}_{4}$ and $\mathrm{H}_{3} \mathrm{PO}_{4}$ after holding $(24 \mathrm{~h})$ in inhibitor preliminary adsorption solution (room $t$ ). The duration of the experiments was $2 \mathrm{~h} . t=20-100^{\circ} \mathrm{C}$.

\begin{tabular}{|c|c|c|c|c|c|c|c|}
\hline \multirow{2}{*}{$\begin{array}{l}\text { Inhibitor preliminary } \\
\text { adsorption solution }\end{array}$} & \multirow{2}{*}{$\begin{array}{l}\text { Solution for } \\
\text { aftereffect } \\
\text { study }\end{array}$} & \multirow{2}{*}{$k / \gamma$} & \multicolumn{5}{|c|}{ Temperature of background solutions, ${ }^{\circ} \mathrm{C}$} \\
\hline & & & 20 & 40 & 60 & 80 & 100 \\
\hline \multirow[t]{2}{*}{-} & $2 \mathrm{M} \mathrm{HCl}$ & $k$ & 1.44 & 4.71 & 33.4 & 204 & 902 \\
\hline & \multicolumn{7}{|c|}{ Without ultrasonic washing } \\
\hline \multirow{2}{*}{$2 \mathrm{M} \mathrm{HCl}+5.0$ mM IFKhAN-92 } & \multirow{2}{*}{$2 \mathrm{M} \mathrm{HCl}$} & $k$ & 0.018 & 0.24 & 0.44 & 1.03 & 554 \\
\hline & & $\gamma$ & 80.0 & 19.6 & 75.9 & 198 & 1.6 \\
\hline \multicolumn{8}{|c|}{ After ultrasound washing } \\
\hline \multirow{2}{*}{ 2M HCl + 5.0 mM IFKhAN-92 } & \multirow{2}{*}{$2 \mathrm{M} \mathrm{HCl}$} & $k$ & 0.16 & 0.39 & 1.2 & 136 & - \\
\hline & & $\gamma$ & 9.0 & 12.1 & 27.8 & 1.5 & - \\
\hline \multirow[t]{2}{*}{-} & $2 \mathrm{M} \mathrm{H}_{2} \mathrm{SO}_{4}$ & $k$ & 6.18 & 27.8 & 65.6 & 252 & 959 \\
\hline & \multicolumn{7}{|c|}{ Without ultrasonic washing } \\
\hline \multirow{2}{*}{$\begin{array}{l}2 \mathrm{M} \mathrm{H}_{2} \mathrm{SO}_{4}+5.0 \mathrm{mM} \text { IFKhAN- } \\
\quad 92+5.0 \mathrm{mM} \mathrm{KNCS}\end{array}$} & \multirow{2}{*}{$2 \mathrm{M} \mathrm{H}_{2} \mathrm{SO}_{4}$} & $k$ & 0.20 & 0.22 & 0.26 & 0.41 & 671 \\
\hline & & $\gamma$ & 30.9 & 126 & 252 & 614 & 1.4 \\
\hline \multicolumn{8}{|c|}{ After ultrasonic washing } \\
\hline \multirow{2}{*}{$\begin{array}{c}2 \mathrm{M} \mathrm{H}_{2} \mathrm{SO}_{4}+5.0 \mathrm{mM} \text { IFKhAN- } \\
\quad 92+5.0 \mathrm{mM} \mathrm{KNCS}\end{array}$} & \multirow{2}{*}{$2 \mathrm{M} \mathrm{H}_{2} \mathrm{SO}_{4}$} & $k$ & 0.25 & 0.66 & 0.97 & 201 & - \\
\hline & & $\gamma$ & 24.7 & 42.1 & 67.6 & 1.3 & - \\
\hline- & $2 \mathrm{M} \mathrm{H}_{3} \mathrm{PO}_{4}$ & $k$ & 6.94 & 11.5 & 40.5 & 193 & 1020 \\
\hline \multicolumn{8}{|c|}{ Without ultrasonic washing } \\
\hline \multirow{2}{*}{$\begin{array}{c}2 \mathrm{M} \mathrm{H}_{3} \mathrm{PO}_{4}+5.0 \mathrm{mM} \text { IFKhAN- } \\
\quad 92+5.0 \mathrm{mM} \mathrm{KNCS}\end{array}$} & \multirow{2}{*}{$2 \mathrm{M} \mathrm{H}_{3} \mathrm{PO}_{4}$} & $k$ & 0.054 & 0.31 & 0.52 & 2.54 & 1130 \\
\hline & & $\gamma$ & 129 & 37.1 & 77.9 & 75.8 & 0.9 \\
\hline \multicolumn{8}{|c|}{ After ultrasonic washing } \\
\hline \multirow{2}{*}{$\begin{array}{c}2 \mathrm{M} \mathrm{H}_{3} \mathrm{PO}_{4}+5.0 \mathrm{mM} \text { IFKhAN- } \\
92+5.0 \mathrm{mM} \mathrm{KNCS}\end{array}$} & \multirow{2}{*}{$2 \mathrm{M} \mathrm{H}_{3} \mathrm{PO}_{4}$} & $k$ & 0.064 & 1.07 & 46.3 & - & - \\
\hline & & $\gamma$ & 108 & 10.7 & 0.89 & - & - \\
\hline
\end{tabular}






Figure 4. XPS spectra of N1s $(a)$ and $\mathrm{Fe} 2 \mathrm{p}(b)$ electrons of surface of steel 3 after holding in 2 $\mathrm{M} \mathrm{H}_{3} \mathrm{PO}_{4}+5 \mathrm{mM}$ IFKhAN-92 $+5 \mathrm{mM} \mathrm{KNCS}\left(24 \mathrm{~h}, 25^{\circ} \mathrm{C}\right)$.

The formation of protective CI layers with molecules bound by chemical bonds is observed on steel 3 in $\mathrm{H}_{3} \mathrm{PO}_{4}$ solutions [250]. Preadsorption of a mixture of IFKhAN-92 and KNCS on the steel surface from an $\mathrm{H}_{3} \mathrm{PO}_{4}$ solution results in the formation of a chemisorbed protective layer thicker than $4 \mathrm{~nm}$. It has a polymeric nature and consists of a complex compound of IFKhAN-92 molecules, Fe(II) cations, and rhodanide anions. The formation of a protective layer is confirmed by the XP spectrum of N1s electrons of the steel surface obtained for nitrogen atoms of different types present in the structure of IFKhAN-92 molecules and rhodanide anions (Figure 4a). Such a layer is not removed from the steel surface in the course of ultrasonic cleaning and manifests a protective aftereffect in $\mathrm{H}_{3} \mathrm{PO}_{4}$ solutions (Table 3). As follows from the Fe $2 p$ spectra (Figure 4b), the metal surface underlying the $\mathrm{CI}$ layer is oxidized to iron oxides. Besides, the oxide phase includes a certain fraction of hydrogen phosphates. This follows from the presence of a peak of $\mathrm{P} 2 p$ electrons in the spectrum of the metal surface.



Figure 5. Protective after-effect of inhibitor layers formed on the surface of chromiumnickel steel $12 \mathrm{Kh} 18 \mathrm{~N} 10 \mathrm{~T}$ in $2 \mathrm{M} \mathrm{HCl}+5 \mathrm{mM}$ IFKhAN-92 $\left(20^{\circ} \mathrm{C}\right)$ in $2 \mathrm{M} \mathrm{HCl}$ solution ( $2 \mathrm{~h}) .1$ - samples after preliminary inhibitor adsorption $(24 \mathrm{~h}), 2$ - samples after preliminary inhibitor adsorption $(24 \mathrm{~h})$ followed by ultrasonic washing, 3 - samples without preliminary inhibitor adsorption. 
Preliminary adsorption of IFKhAN-92 on the surface of $12 \mathrm{Kh} 18 \mathrm{~N} 10 \mathrm{~T}$ steel from $\mathrm{HCl}$ solution results in the formation of a polymolecular protective layer of the organic CI chemically bound to the phase of $\mathrm{Fe}, \mathrm{Cr}$, and $\mathrm{Ni}$ oxides and hydroxides adjoining the metallic phase [251, 252]. The lower part of the polymolecular protective layer of the organic CI consists of a polymeric complex formed by IFKhAN-92 molecules, metal cations (Fe, $\mathrm{Cr}$, and $\mathrm{Ni}$ ), and chloride anions, while the outer part consists of physically adsorbed IFKhAN92 molecules. The weakly bound CI layers are removed from the metal surface in the course of its ultrasonic cleaning in $\mathrm{HCl}$ solution. The $\mathrm{CI}$ layer (about 3 monolayers) remaining on the metal surface manifests a protective aftereffect in $2 \mathrm{M} \mathrm{HCl}$ at $t$ up to $80^{\circ} \mathrm{C}$ (Figure 5). This points to the chemical character of interaction between the organic CI within the layer of its complex polymer and the surface of the oxide-hydroxide phase. The formulation of IFKhAN-92 + urotropine (molar ratio 1:4) is more efficient in the protection of $12 \mathrm{Kh} 18 \mathrm{~N} 10 \mathrm{~T}$ steel. Studies of the surface composition of samples pretreated in such solutions show that urotropine, alongside with IFKhAN-92, is included in the chemisorbed organic CI layer. The amount of urotropine in the chemisorbed layer is in the range of 20$30 \%$. The thickness of the protective layer formed in the presence of this CI formulation is smaller than in the case of individual IFKhAN-92. Strong protective properties at smaller thicknesses point to its more compact structure. This layer is strongly bound to the metal surface and is not removed after ultrasonic cleaning. The strong adhesion of IFKhAN-92 molecules and urotropine in the organic CI layer is explained by their chemical cross-linking that is initiated by a product on urotropine hydrolysis, formaldehyde.

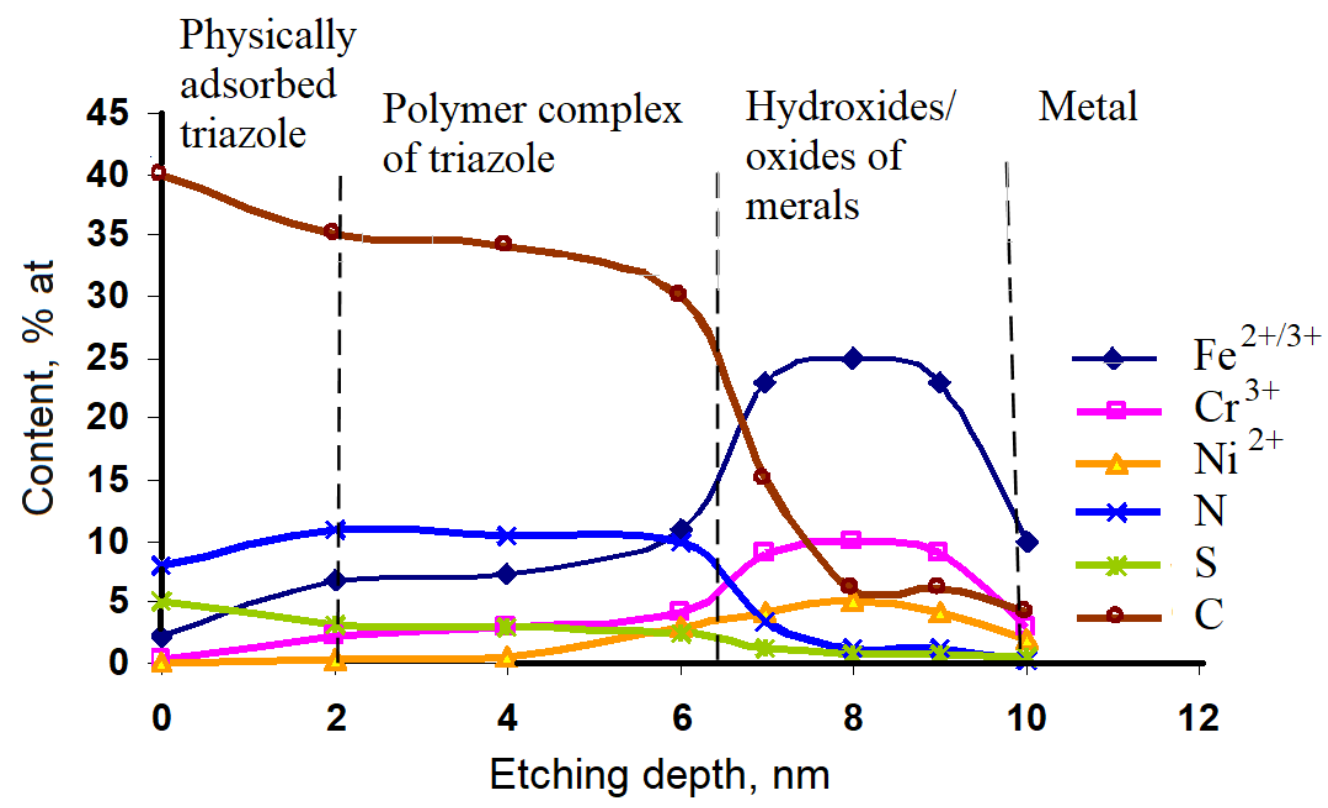

Figure 6. Distribution of elements upon etching by argon ions of the surface of $12 \mathrm{Kh} 18 \mathrm{~N} 10 \mathrm{~T}$ steel exposed in $2 \mathrm{M} \mathrm{H}_{2} \mathrm{SO}_{4}+4.0 \mathrm{mM}$ IFKhAN-92 $+1.0 \mathrm{mM} \mathrm{KNCS}\left(24 \mathrm{~h}, 20^{\circ} \mathrm{C}\right)$. 




Figure 7. Distribution of elements upon etching by argon ions of the surface of $12 \mathrm{Kh} 18 \mathrm{~N} 10 \mathrm{~T}$ steel exposed in $2 \mathrm{M} \mathrm{H}_{2} \mathrm{SO}_{4}+2.5 \mathrm{mM}$ IFKhAN-92 $+2.5 \mathrm{mM} \mathrm{KI}\left(24 \mathrm{~h}, 20^{\circ} \mathrm{C}\right)$.

We observed the formation of protective layers with a similar structure on a chromiumnickel steel protected in $\mathrm{H}_{2} \mathrm{SO}_{4}$ solutions by composite CIs: IFKhAN-92 + KNCS (Figure 6) and IFKhAN-92 + KI (Figure 7) [253, 254]. Protective layers of organic CI are chemically bound to the phases of $\mathrm{Fe}, \mathrm{Cr}$, and $\mathrm{Ni}$ hydroxides and oxides adjoining the metallic phase. The lower phase of the polymolecular protective layer of organic CI consists of a polymer complex formed by IFKhAN-92 molecules and metal (Fe, $\mathrm{Cr}$, and $\mathrm{Ni}$ ) cations, while the outer part is composed of physically adsorbed IFKhAN-92 molecules easily removed from the metal surface by sonication. In the case of the IFKhAN-92 + KNCS formulation, the polymer complex additionally contains rhodanide anions. The inclusion of bridging rhodanide anions into the layer provides the possibility of its thickening owing to the appearance of additional variants of coordination interaction of its particles. These protective layers are definitely close in structure to the polymeric layers of IFKhAN-92 formed on steel 3 in an $\mathrm{H}_{3} \mathrm{PO}_{4}+$ IFKhAN-92 + KNCS system. The protective layer formed by the formulation of IFKhAN-92 + KNCS has a thicker organic part but provides poorer protection than the layers formed by the formulation of IFKhAN-92 + KI. The probable cause lies in the composition and structure of the metal hydroxide/oxide layer. In the presence of KI, it is enriched with chromium that should improve its protective properties.

It was shown for 1,2,4-triazole derivatives that the efficient inhibition of metal corrosion by NFHCs in acid media is due to their ability to form strongly bound polymolecular protective layers on a metal surface. A particular feature of such layers is that the underlying organic CI layer is chemically bound to the metal surface. The CI layers above this layer contain molecules that are bound with each other either physically or chemically. 
The maximum protective effects are provided by chemically bound CI layers. An oxidehydroxide layer appears quite often between an organic CI layer and a metal surface. This additional layer makes its own contribution to the protective effect of the organic polymolecular layer. The chemical interaction of molecules in the protective polymeric layer is based on the coordination interaction of complexing agents (metal cations formed in the course of corrosion of the metal being protected) and ligands (molecules of nitrogencontaining heterocycles). The strongest protective layers are formed if the nitrogencontaining heterocycle is a bidentate ligand.

The role of additives enhancing the protective effect of NFHCs is to impart new properties to the protective layer in order to favor its best protective ability. The mechanisms of action of such additives are quite diverse. In fact, in the case of steel protection in acid solutions by a substituted triazole (IFKhAN-92), the rhodanide anion accelerates the adsorption of the organic CI on the metal. They simultaneously participate in the formation of a polymolecular protective film and are included in the structure of the polymeric complex. In the case of protection of alloys with a complex composition (stainless steels), iodide anions can change the composition of the oxide-hydroxide phase existing between the metal and the organic part of the protective layer. The urotropine additive is not only incorporated in the structure of a protective organic CI layer but also promotes the additional chemical binding of its constituent molecules of nitrogen-containing heterocycles.

There is vast literature data on the electrochemical behavior of NFHCs on metals in acid solutions, generally at temperatures close to room temperature. The results of voltammetric measurements show that these compounds efficiently inhibit cathodic and anodic reactions on metals. According to EIS studies, the addition of such CIs to a corrosive medium is accompanied by a decrease in the capacitance of the electric double layer on metals and an increase in the polarization resistance. The voltammetry and EIS data are also in good agreement with the corrosion rates of metal samples obtained by mass loss measurements.

Of special interest are electrochemical data corresponding to a wide range of temperatures, $t=20-100^{\circ} \mathrm{C}$. Formulations based on a substituted triazole (IFKhAN-92) can noticeably inhibit the electrode reactions of low-carbon steels in solutions of $\mathrm{HCl}, \mathrm{H}_{2} \mathrm{SO}_{4}$ [220], and $\mathrm{H}_{3} \mathrm{PO}_{4}$ [255] and of chromium-nickel steels in solutions of $\mathrm{HCl}$ [140] and $\mathrm{H}_{2} \mathrm{SO}_{4}$ [143] even at $t$ close to $100^{\circ} \mathrm{C}$. A similar pattern was observed for low-carbon steel in acetic and formic acid solutions at $t=100^{\circ} \mathrm{C}$ [226], which is largely due to the formation of protective CI layers on metals by NFHCs. 


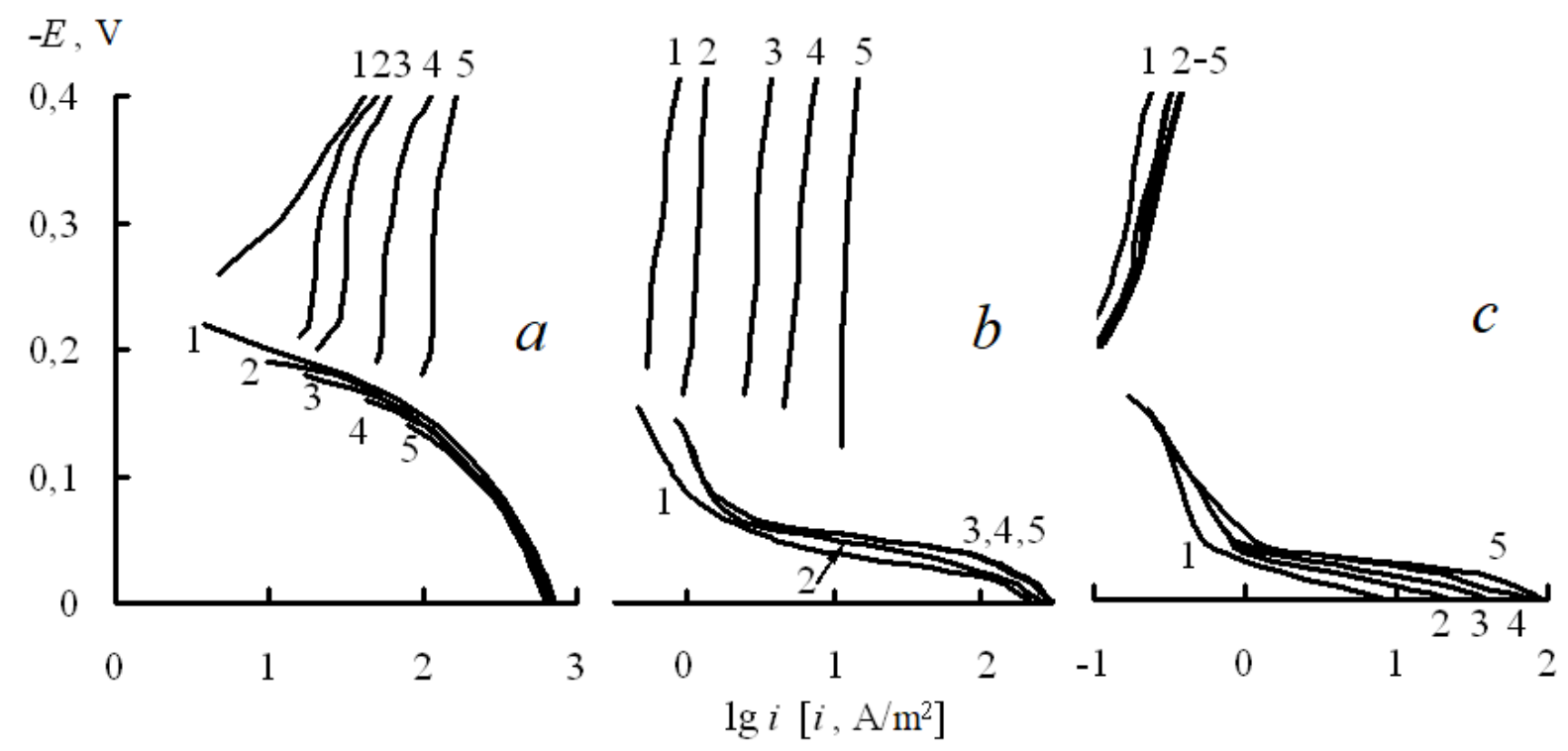

Figure 8. Polarization curves of the steel 3 disk $(n=460 \mathrm{rpm})$ in $2.0 \mathrm{M} \mathrm{H}_{3} \mathrm{PO}_{4}(a)$, inhibited by $0.5 \mathrm{mM}$ IFKhAN-92 + 0.5 mM KNCS $(b)$ and 5.0 mM IFKhAN-92 + 0.5 mM KNCS $(c)$, in the presence of $\mathrm{FePO}_{4}(\mathrm{~mol} / \mathrm{L}): 1-0.00,2-0.01,3-0.02,4-0.05,5-0.10 . t=25^{\circ} \mathrm{C}$.

A more complex mechanism of the protective action of NFHC-based composite CIs is implemented in the case of steel corrosion in acid media containing $\mathrm{Fe}(\mathrm{III})$ salts. Its understanding became possible owing to the employment of a complex of electrochemical research techniques: potentiometry, cyclic voltammetry on a platinum electrode, and voltammetry on a steel disk electrode. They were used to study both acid solutions containing $\mathrm{Fe}$ (III) salts and a (steel-acid solution) corrosion system. In $\mathrm{H}_{3} \mathrm{PO}_{4}$ solutions containing $\mathrm{FePO}_{4}$, the inhibitor formulation of IFKhAN-92 + KNCS efficiently inhibits the corrosion of low-carbon steels. In contrast, in $\mathrm{H}_{2} \mathrm{SO}_{4}$ solutions containing $\mathrm{Fe}_{2}\left(\mathrm{SO}_{4}\right)_{3}$ and in $\mathrm{HCl}$ solutions containing $\mathrm{FeCl}_{3}$, this $\mathrm{CI}$ has nearly no protective effect. The reason is that this CI efficiently inhibits the electrode reactions on steel only in $\mathrm{H}_{3} \mathrm{PO}_{4}$ solutions containing $\mathrm{FePO}_{4}$ (Figure 8) [228].

In this system, the cathodic reaction of steel involves two partial processes, namely, the reduction of $\mathrm{H}^{+}$and $\mathrm{Fe}^{3+}$. The first reaction occurs in the kinetic control zone, while the second one is controlled by diffusion. A rotating disk electrode is used to study electrochemical processes occurring with diffusion control. One can see that the rate of the cathodic reaction in an $\mathrm{H}_{3} \mathrm{PO}_{4}$ solution containing $\mathrm{FePO}_{4}$ significantly depends on the disk electrode rotation rate (Figure 9). In a static solution, the cathodic reaction of steel is almost totally determined by the reduction of $\mathrm{H}^{+}$. Here, the rise on the cathodic current due to the electrode rotation is governed almost completely by the following reaction:

$$
\mathrm{Fe}^{3+}+\mathrm{e}=\mathrm{Fe}^{2+} .
$$




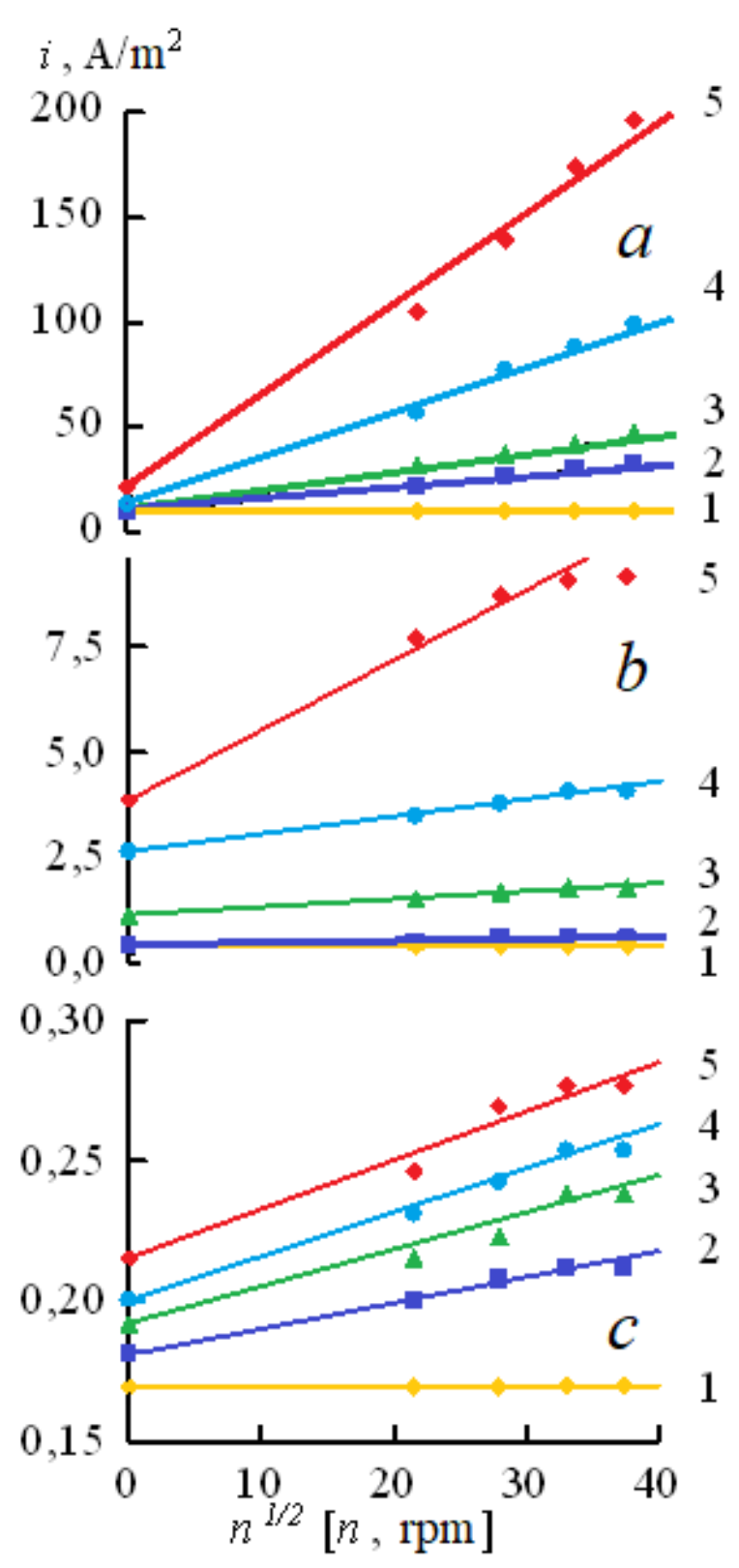

Figure 9. Dependence of the cathodic current density at $E=-0.30 \mathrm{~V}$ on the square root of the rotational speed of the disk electrode (steel 3) in $2.0 \mathrm{M} \mathrm{H}_{3} \mathrm{PO}_{4}(a)$, inhibited by $0.5 \mathrm{mM}$ IFKhAN-92 + 0.5 mM KNCS $(b)$ and $5.0 \mathrm{mM}$ IFKhAN-92 + 0.5 mM KNCS $(c)$, in the presence of $\mathrm{FePO}_{4}(\mathrm{~mol} / \mathrm{L}): 1-0.00,2-0.01,3-0.02,4-0.05,5-0.10 . t=25^{\circ} \mathrm{C}$.

In a similar medium inhibited by IFKhAN $-92+\mathrm{KNCS}$, the cathodic current is much lower both in static and dynamic solutions. Moreover, the response cathodic current to a variation in the hydrodynamic mode of the solution is very weak. One can see that the IFKhAN-92 + KNCS formulation possesses a unique ability not only to suppress the reduction of $\mathrm{H}^{+}$but also to inhibit the reduction of $\mathrm{Fe}^{3+}$ considerably.

The protective layers formed by the IFKhAN-92 + KNCS formulation on a steel surface can provide efficient inhibition of $\mathrm{Fe}^{3+}$ reduction in $\mathrm{H}_{3} \mathrm{PO}_{4}$ solutions in the diffusion mode. This effect is, however, absent due to some reasons in $\mathrm{H}_{2} \mathrm{SO}_{4}$ and $\mathrm{HCl}$ solutions containing 
Fe(III) salts. It has been shown using potentiometry [256, 257] and cyclic voltammetry $[258,259]$ that, as opposed to $\mathrm{H}_{2} \mathrm{SO}_{4}$ and $\mathrm{HCl}$ solutions, the oxidative ability of $\mathrm{Fe}$ (III) cations was lower in $\mathrm{H}_{3} \mathrm{PO}_{4}$ solutions owing to their complexation by phosphate anions. In addition, the mobility of $\mathrm{Fe}(\mathrm{III})$ cations decreased in $\mathrm{H}_{3} \mathrm{PO}_{4}$ solutions considerably $[258,259]$, which significantly affected the process of $\mathrm{Fe}(\mathrm{III})$ reduction occurring in the diffusion mode. Importantly, the presence of $\mathrm{H}_{3} \mathrm{PO}_{4}$ in $\mathrm{H}_{2} \mathrm{SO}_{4}$ and $\mathrm{HCl}$ solutions containing $\mathrm{Fe}(\mathrm{III})$ salts also removes the oxidative ability and mobility of $\mathrm{Fe}(\mathrm{III})$ cations in these solutions. This effect allows one to develop inhibited solutions of acid mixtures $\left(\mathrm{H}_{2} \mathrm{SO}_{4}+\right.$ $\mathrm{H}_{3} \mathrm{PO}_{4}$ and $\mathrm{HCl}+\mathrm{H}_{3} \mathrm{PO}_{4}$ ) that are stable to the accumulation of $\mathrm{Fe}(\mathrm{III})$, as discussed above.

A study on $\mathrm{H}_{2} \mathrm{SO}_{4}+\mathrm{H}_{3} \mathrm{PO}_{4}$ and $\mathrm{HCl}+\mathrm{H}_{3} \mathrm{PO}_{4}$ solutions containing $\mathrm{Fe}(\mathrm{III})$ salts demonstrated that the three-component formulation IFKhAN-92 + KNCS + urotropine had a higher efficiency in steel protection in these media than the binary formulation of IFKhAN$92+\mathrm{KNCS}$. Here, the role of urotropine in this effect remained unclear. A potentiometric study of $\mathrm{H}_{2} \mathrm{SO}_{4}+\mathrm{H}_{3} \mathrm{PO}_{4}$ and $\mathrm{HCl}+\mathrm{H}_{3} \mathrm{PO}_{4}$ solutions containing $\mathrm{Fe}(\mathrm{III})$ salts showed that urotropine present in the solutions additionally bound $\mathrm{Fe}(\mathrm{III})$ cations into complex compounds, which resulted in an additional decrease in the oxidizing ability of these systems [260].

Thus, the development of inhibited acid solutions that are resistant to the accumulation of $\mathrm{Fe}(\mathrm{III})$ salts is possible only by combining the ability of $\mathrm{H}_{3} \mathrm{PO}_{4}$-containing acid solutions to bind $\mathrm{Fe}$ (III) cations into complex compounds with lower corrosivity towards steels and the unique properties of the IFKhAN-92 + KNCS + urotropine formulation. This formulation not only forms a polymolecular protective CI layer of IFKhAN-92 molecules and rhodanide anions on a metal surface that efficiently suppresses Fe(III) reduction, but also, owing to the presence of urotropine, additionally decreases the oxidative ability of $\mathrm{Fe}$ (III) present in the corrosive medium.

\section{Conclusion}

So far, ample literature data have been accumulated regarding the protection of metals (predominantly steels) in NFHC-containing acid solutions. The studied CIs are both simple and complex heterocyclic systems including aromatic, unsaturated, and saturated ones. In these CI groups, the highest protective effect is often provided by heterocycle derivatives with bulky substituents. NFHCs tend to be adsorbed on metal surfaces from mineral acid solutions. One can assume with high probability, judging by the ( $\left.-\Delta G_{\text {ads }}\right)$ values of these CIs on the metal surfaces, that some of them can be chemically bound to the metals. These compounds adsorbed on a metal surface inhibit the cathodic and anodic reactions, which ultimately suppresses its corrosion. An important feature of such compounds is their ability to form polymolecular protective layers on the metal surface, which allows these compounds to provide the most efficient protection.

An acceptable practical form of application of these compounds is to use them in order to develop composite CIs. NFHC-based formulations can be used for metal protection in acid media where a heterocycle alone is characterized by low efficiency. NFHC formulations 
with some simple compounds (KI, KBr, KNCS, urotropine) can form protective polymolecular CI layers on a metal surface, where NFHC molecules are chemically bound with each other and with the metal surface being protected. They manifest high protection ability owing to the characteristic composition and structure of these layers. Owing to the ability to form such structures on the metal surface, composite NFHC-based CIs can protect various steels in very corrosive high-temperature solutions of mineral acids ( $t$ up to $200^{\circ} \mathrm{C}$ ) and in $\mathrm{Fe}(\mathrm{III})$-containing acid media.

\section{Acknowledgements}

The study was carried out within the framework of the Program of Basic Scientific Research of the State Academies of Sciences for 2013-2020 (Subject: "Development of the fundamental scientific basis of the protective effect of inhibitors of metal corrosion in gas and condensed media, nanocomposites, paintwork and conversion coatings", state registration number AAAA-A18-118121090043-0).

\section{References}

1. Yu.I. Kuznetsov and L.P. Kazansky, Physicochemical aspects of metal protection by azoles as corrosion inhibitors, Russ. Chem. Rev., 2008, 77, no. 3, 219-232. doi: 10.1070/RC2008v077n03ABEH003753

2. Yu.I. Kuznetsov, Triazoles as a class of multifunctional corrosion inhibitors. A review. Part I. 1,2,3-Benzotriazole and its derivatives. Copper, zinc and their alloys, Int. J. Corros. Scale Inhib., 2018, 7, no. 3, 271-307. doi: 10.17675/2305-6894-2018-7-3-1

3. Yu.I. Kuznetsov, Triazoles as a class of multifunctional corrosion inhibitors. A review. Part II. 1,2,3-benzotriazole, its derivatives. Iron and steels, Int. J. Corros. Scale Inhib., 2020, 9, no. 2, 780-811. doi: 10.17675/2305-6894-2020-9-3-1

4. Yu.I. Kuznetsov, Triazoles as a class of multifunctional corrosion inhibitors. A review. Part III. 1,2,3-Benzotriazole and its derivatives. Aluminum alloys, Int. J. Corros. Scale Inhib., 2020, 9, no. 3, 1142-1168. doi: 10.17675/2305-6894-2020-9-4-1

5. I. Merimi, R. Touzani, A. Aouniti, A. Chetouani and B. Hammouti, Pyrazole derivatives efficient organic inhibitors for corrosion in aggressive media: A comprehensive review, Int. J. Corros. Scale Inhib., 2020, 9, no. 4, 1237-1260. doi: 10.17675/2305-6894-20209-4-4

6. R. Salim, E. Ech-chihbi, H. Oudda, F. El Hajjaji, M. Taleb and S. Jodeh, A Review on the Assessment of Imidazo[1,2-a]pyridines As Corrosion Inhibitor of Metals, J. Bio Tribo Corros., 2019, 5, 14. doi: 10.1007/s40735-018-0207-3

7. A. Mishra, C. Verma, M.A. Quraishi and E.E. Ebenso, Imidazoles as highly effective heterocyclic corrosion inhibitors for metals and alloys in aqueous electrolytes: A review, J. Taiwan Inst. Chem. Eng., 2020, 114, 341-358. doi: 10.1016/j.jtice.2020.08.034

8. Ya.G. Avdeev and Yu.I. Kuznetsov, Physicochemical aspects of inhibition of acid corrosion of metals by unsaturated organic compounds, Russ. Chem. Rev., 2012, 81, no. 12, 1133-1145. doi: 10.1070/RC2012v081n12ABEH004292 
9. Ya.G. Avdeev, Nitrogen-containing six-membered heterocyclic compounds as corrosion inhibitors for metals in solutions of mineral acids - A review, Int. J. Corros. Scale Inhib., 2018, 7, no. 4, 460-497. doi: 10.17675/2305-6894-2018-7-4-1

10. Ya.G. Avdeev and Yu.I. Kuznetsov, Inhibitory protection of steels in acid solutions under high-temperature corrosion conditions. A review. Part 3, Int. J. Corros. Scale Inhib., 2020, 9, no. 4, 1194-1236. doi: 10.17675/2305-6894-2020-9-4-3

11. K.R. Ansari, D.S. Chauhan, A. Singh, V.S. Saji and M.A. Quraishi, Corrosion Inhibitors for Acidizing Process in Oil and Gas Sectors, in Corrosion Inhibitors in the Oil and Gas Industry, Eds.: V.S. Saji and S.A. Umoren, Wiley-VCH Verlag GmbH \& Co. KGaA, Weinheim, Germany, 2020, 153-176. doi: 10.1002/9783527822140.ch6

12. M.A. Quraishi, D.S. Chauhan and V.S. Saji, Heterocyclic corrosion inhibitors for acid environments, in Heterocyclic Organic Corrosion Inhibitors, Eds.: M.A. Quraishi, D.S. Chauhan and V.S. Saji, Elsevier Inc., 2020, 87-131. doi: 10.1016/B978-0-12818558-2.00004-7

13. K. Rasheeda, Vijaya D.P. Alva, P.A. Krishnaprasad and S. Samshuddin, Pyrimidine derivatives as potential corrosion inhibitors for steel in acid medium - An overview, Int. J. Corros. Scale Inhib., 2018, 7, no. 1, 48-61. doi: 10.17675/2305-6894-2018-7-1-5

14. M.A. Quraishi, D.S. Chauhan and V.S. Saji, Heterocyclic corrosion inhibitors for sweet and sour environments, in Heterocyclic Organic Corrosion Inhibitors, Eds.: M.A. Quraishi, D.S. Chauhan and V.S. Saji, Elsevier Inc., 2020, 133-157. doi: 10.1016/B978-0-12-818558-2.00005-9

15. I.B. Obot, I.B. Onyeachu, S.A. Umoren, M.A. Quraishi, A.A. Sorour, T. Chen, N. Aljeaban and Q. Wang, High temperature sweet corrosion and inhibition in the oil and gas industry: Progress, challenges and future perspectives, J. Pet. Sci. Eng., 2020, 185, 106469. doi: 10.1016/j.petrol.2019.106469

16. Yu.I. Kuznetsov, A.A. Chirkunov and A.M. Semiletov, Progress in the inhibition of metal corrosion and the prospects of its use in the oil and gas industry, Int. J. Corros. Scale Inhib., 2019, 8, no. 4, 850-881. doi: 10.17675/2305-6894-2019-8-4-5

17. R. Kashkovskiy, K. Strelnikova and A. Fedotova, Application of electrochemical impedance spectroscopy to study hydrogen sulphide corrosion of steel and its inhibition: a review, Corros. Eng., Sci. Technol., 2019, 54, no. 6, 493-515. doi: 10.1080/1478422X.2019.1619979

18. M.A. Quraishi, D.S. Chauhan and V.S. Saji, Heterocyclic corrosion inhibitors for neutral environments, in Heterocyclic Organic Corrosion Inhibitors, Eds.: M.A. Quraishi, D.S. Chauhan and V.S. Saji, Elsevier Inc., 2020, 159-193. doi: 10.1016/B978-0-12$\underline{818558-2.00006-0}$

19. M.A. Quraishi, D.S. Chauhan and V.S. Saji, Heterocyclic corrosion inhibitors for alkaline environments, in Heterocyclic Organic Corrosion Inhibitors, Eds.: M.A. Quraishi, D.S. Chauhan and V.S. Saji, Elsevier Inc., 2020, 195-209. doi: $\underline{10.1016 / \mathrm{B} 978-0-12-818558-2.00007-2}$ 
20. M.A. Quraishi, D.S. Chauhan and V.S. Saji, Heterocyclic corrosion inhibitors for vaporphase environments, in Heterocyclic Organic Corrosion Inhibitors, Eds.: M.A. Quraishi, D.S. Chauhan and V.S. Saji, Elsevier Inc., 2020, 211-223. doi: 10.1016/B978-0-12818558-2.00008-4

21. Standard ISO 8044-1989.

22 V.I. Ivanskiy, Khimiya geterotsiklicheskikh soedinenii: Ucheb. posobie dlya universitetov (Chemistry of Heterocyclic Compounds: Manual for Universities), Vysshaya shkola, Moscow, 1978, 559 pp. (in Russian).

23. R.M. Hudson, T.J. Butler and C.J. Warning, The effect of pyrrole-halide mixtures in inhibiting the dissolution of low-carbon steel in sulphuric acid, Corros. Sci., 1977, 17, no. 7, 571-581. doi: 10.1016/S0010-938X(77)80003-6

24. O. Krim, M. Bouachrine, B. Hammouti, A. Elidrissi and M. Hamidi, 2,5-Difuryl-NMethylpyrrole as Corrosion Inhibitor for Steel in $1 \mathrm{M} \mathrm{HCl}$, Port. Electrochim. Acta, 2008, 26, no. 3, 283-289. doi: 10.4152/pea.200803283

25. A. Zarrouk, T. Lakhlifi, H. Vezin and F. Bentiss, New $1 H$-pyrrole-2,5-dione derivatives as efficient organic inhibitors of carbon steel corrosion in hydrochloric acid medium: Electrochemical, XPS and DFT studies, Corros. Sci., 2015, 90, 572-584. doi: 10.1016/j.corsci.2014.10.052

26. A. Louroubi, A. Nayad, A. Hasnaoui, I. Hdoufane, R. Idouhli, M. Saadi, L. El Ammari, A. Abdessalam, M. Berraho, L. El Firdoussi and M. Ait Ali, Synthesis, structural characterization, theoretical studies and corrosion inhibition of a new pyrrole derivative:1-(1-benzyl-4-(4-chlorophenyl)-2,5-dimethyl-1H-pyrrol-3-yl)ethanone, $J$. Mol. Struct., 2019, 1189, 240-248. doi: 10.1016/j.molstruc.2019.03.095

27. A. Charles, K. Sivaraj and S. Thanikaikarasan, Synthesis, characterization and corrosion studies of Schiff bases derived from pyrrole-2-carbaldehyde, Mater. Today: Proc., 2020, 33, 3135-3138. doi: 10.1016/j.matpr.2020.03.741

28. C.B. Verma, E.E. Ebenso, I. Bahadur, I.B. Obot and M.A. Quraishi, 5-(Phenylthio)-3Hpyrrole-4-carbonitriles as effective corrosion inhibitors for mild steel in $1 \mathrm{M} \mathrm{HCl}$ : Experimental and theoretical investigation, J. Mol. Liq., 2015, 212, 209-218. doi: 10.1016/j.molliq.2015.09.009

29. C.B. Verma and M.A. Quraishi, 2-Amino-3-methyl-3-(4-nitrophenyl)-5-(phenylthio)$3 \mathrm{H}$-pyrrole-4-carbonitrile as effective corrosion inhibitor for mild steel in $1 \mathrm{M} \mathrm{HCl}$ : Thermodynamical, electrochemical, surface and theoretical calculation, Ain Shams Eng. J., 2016, 7, 1-9. doi: 10.1016/j.asej.2015.11.017

30. K. Aramaki, Inhibition Effect of N-Substituted Cyclic Imines on Iron Corrosion, Denki Kagaku oyobi Kogyo Butsuri Kagaku, 1972, 40, no. 8, 566-571. doi: 10.5796/kogyobutsurikagaku.40.566

31. M. Bouklah, A. Ouassini, B. Hammouti and A. El Idrissi, Corrosion inhibition of steel in sulphuric acid by pyrrolidine derivatives, Appl. Surf. Sci., 2006, 252, 2178-2185. doi: $\underline{10.1016 / j . a p s u s c .2005 .03 .177}$ 
32. M. Bouklah, O. Krim, M. Messali, B. Hammouti, A. Elidrissi and I. Warad, A pyrrolidine phosphonate derivative as corrosion inhibitor for steel in $\mathrm{H}_{2} \mathrm{SO}_{4}$ solution, Pharma Chem., 2011, 3, no. 5, 283-293.

33. M. Jeeva, M. susai Boobalan and G.V. Prabhu, Adsorption and anticorrosion behavior of 1-((pyridin-2-ylamino)(pyridin-4-yl)methyl)pyrrolidine-2,5-dione on mild steel surface in hydrochloric acid solution, Res. Chem. Intermed., 2018, 44, 425-454. doi: 10.1007/s11164-017-3112-3

34. J. Haque, M.A. Jafar Mazumder, M.A. Quraishi, S.A. Ali and N.A. Aljeaban, Pyrrolidine-based quaternary ammonium salts containing propargyl and hydrophobic C12 and C-16 alkyl chains as corrosion inhibitors in aqueous acidic media, J. Mol. Liq., 2018, 320, 114473. doi: 10.1016/j.molliq.2020.114473

35. N.I. Podobaev and Ya.G. Avdeev, A Review of Acetylene Compounds as Inhibitors of Acid Corrosion of Iron, Prot. Met., 2004, 40, no. 1, 7-13. doi: 10.1023/B:PROM.0000013105.48781.86

36. Ya.G. Avdeev and Yu.I. Kuznetsov, Inhibitory protection of steels from hightemperature corrosion in acid solutions. A review. Part 2., Int. J. Corros. Scale Inhib., 2020, 9, no. 3, 867-902. doi: 10.17675/2305-6894-2020-9-3-5

37. N.I. Podobaev and Ya.G. Avdeev, Effect of the Molecule Structure of Acetylene Compounds on the Kinetics of the Electrode Reactions of Iron in Hydrochloric and Sulfuric Acid, Prot. Met., 2002, 38, no. 1, 45-50. doi: 10.1023/A:1013852801262

38. Q.-Q. Liao, Z.-W. Yue, Z.-W. Zhu, Y. Wang, Y. Zhang, G.-D. Zhou and Q. Zhou, Corrosion Inhibition Effect of Self-Assembled Monolayers of Ammonium Pyrrolidine Dithiocarbamate on Copper, Acta Phys.-Chim. Sin., 2009, 25, no. 8, 1655-1661. doi: 10.3866/PKU.WHXB20090811

39. M. Bagherzadeh and F. Jaberinia, Electrochemical study of Monel alloy corrosion in hydrochloric acid solution and pyrrolidine dithiocarboxylate self-assembled monolayers as its corrosion protector, J. Alloys Compd., 2018, 750, 677-686. doi: 10.1016/j.jallcom.2018.04.074

40. S.A. Umoren and I.B. Obot, Polyvinylpyrollidone and polyacrylamide as corrosion inhibitors for mild steel in acidic medium, Surf. Rev. Lett., 2008, 15, no. 3, 277-286. doi: 10.1142/S0218625X08011366

41. L.A. Al Juhaiman, Polyvinyl pyrrolidone as a Corrosion Inhibitor for Carbon Steel in HCl, Int. J. Electrochem. Sci., 2016, 11, 2247-2262.

42. G. Achary, Y.A. Naik, S.V. Kumar, T.V. Venkatesha and B.S. Sherigara, An electroactive co-polymer as corrosion inhibitor for steel in sulphuric acid medium, Appl. Surf. Sci., 2008, 254, 5569-5573. doi: 10.1016/j.apsusc.2008.02.103

43. S.A. Umoren, Synergistic inhibition effect of polyethylene glycol-polyvinyl pyrrolidone blends for mild steel corrosion in sulphuric acid medium, J. Appl. Polym. Sci., 2011, 119, 2072-2084. doi: 10.1002/app.32922 
44. R. Karthikaiselvi and S. Subhashini, The water soluble composite poly(vinylpyrrolidone-methylaniline): A new class of corrosion inhibitors of mild steel in hydrochloric acid media, Arabian J. Chem., 2017, 10, S627-S635. doi: 10.1016/j.arabjc.2012.10.024

45. E.A. Khamis, A. Hamdy and R.E. Morsi, Magnetite nanoparticles/polyvinyl pyrrolidone stabilized system for corrosion inhibition of carbon steel, Egypt. J. Pet., 2018, 27, 919926. doi: 10.1016/j.ejpe.2018.02.001

46. M. Abdallah, I. Zaafarany, K.S. Khairou and M. Sobhi, Inhibition of Carbon Steel Corrosion by Iron(III) and Imidazole in Sulfuric Acid, Int. J. Electrochem. Sci., 2012, 7, 1564-1579.

47. S.A. Mangai and S. Ravi, Comparative Corrosion Inhibition Effect of Imidazole Compounds and of Trichodesma indicum (Linn) R. Br. on C38 Steel in $1 \mathrm{M} \mathrm{HCl}$ Medium, J. Chem., 2013, 2013, 527286. doi: $10.1155 / 2013 / 527286$

48. C. Pragathiswaran, P. Ramadevi and K.K. Kumar, Imidazole and $\mathrm{Al}^{3+}$ nano material as corrosion inhibitor for mild steel in hydrochloric acid solutions, Mater. Today: Proc., 2020, 37, 2912-2916. doi: 10.1016/j.matpr.2020.08.671

49. A. Ismail, H.M. Irshad, A. Zeino and I.H. Toor, Electrochemical Corrosion Performance of Aromatic Functionalized Imidazole Inhibitor Under Hydrodynamic Conditions on API X65 Carbon Steel in 1M HCl Solution, Arabian J. Sci. Eng., 2019, 44, 5877-5888. doi: $\underline{10.1007 / \mathrm{s} 13369-019-03745-6}$

50. O. Krim, S. Jodeh, M. Messali, B. Hammouti, A. Elidrissi, K. Khaled, R. Salghi and H. Lgaz, Synthesis, Characterization and Corrosion Protection Properties of Imidazole Derivatives on Mild Steel in 1.0 M HCl, Port. Electrochim. Acta, 2016, 34, no. 3, $213-$ 229. doi: $10.4152 /$ pea.201603213

51. M. Yadav, P.N. Yadav and U. Sharma, Substituted imidazoles as corrosion inhibitors for N80 steel in hydrochloric acid, Indian J. Chem. Technol., 2013, 20, no. 6, 363-370.

52. M. Ouakki, M. Galai, M. Rbaa, A.S. Abousalem, B. Lakhrissi, M. Ebn Touhami and M. Cherkaoui, Electrochemical, thermodynamic and theoretical studies of some imidazole derivatives compounds as acid corrosion inhibitors for mild steel, J. Mol. Liq., 2020, 319, 114063. doi: 10.1016/j.molliq.2020.114063

53. M. Ouakki, M. Galai, M. Rbaa, A.S. Abousalem, B. Lakhrissi, E.H. Rifi and M. Cherkaoui, Quantum chemical and experimental evaluation of the inhibitory action of two imidazole derivatives on mild steel corrosion in sulphuric acid medium, Heliyon, 2019, 5, e02759. doi: 10.1016/j.heliyon.2019.e02759

54. T. Zhou, J. Yuan, Z. Zhang, X. Xin and G. Xu, The comparison of imidazolium Gemini surfactant $\left[\mathrm{C}_{14}-4-\mathrm{C}_{14} \mathrm{im}\right] \mathrm{Br}_{2}$ and its corresponding monomer as corrosion inhibitors for A3 carbon steel in hydrochloric acid solutions: Experimental and quantum chemical studies, Colloids Surf., A, 2019, 575, 57-65. doi: 10.1016/j.colsurfa.2019.05.004 
55. V. Srivastava, J. Haque, C. Verma, P. Singh, H. Lgaz, R. Salghi and M.A. Quraishi, Amino acid based imidazolium zwitterions as novel and green corrosion inhibitors for mild steel: Experimental, DFT and MD studies, J. Mol. Liq., 2017, 244, 340-352. doi: 10.1016/j.molliq.2017.08.049

56. J. Wang, D. Liu, S. Cao, S. Pan, H. Luo, T. Wang, H. Ding, B.B. Mamba and J. Gui, Inhibition effect of monomeric/polymerized imidazole zwitterions as corrosion inhibitors for carbon steel in acid medium, J. Mol. Liq., 2020, 312, 113436. doi: 10.1016/j.molliq.2020.113436

57. J. Haque, V. Srivastava, M.A. Quraishi, D.S. Chauhan, H. Lgaz and I.-M. Chung, Polar group substituted imidazolium zwitterions as eco-friendly corrosion inhibitors for mild steel in acid solution, Corros. Sci., 2020, 172, 108665. doi: 10.1016/j.corsci.2020.108665

58. N.V. Likhanova, M.A. Dominguez-Aguilar, O. Olivares-Xometl, N. Nava-Entzana, E. Arce and H. Dorantes, The effect of ionic liquids with imidazolium and pyridinium cations on the corrosion inhibition of mild steel in acidic environment, Corros. Sci., 2010, 52, 2088-2097. doi: 10.1016/j.corsci.2010.02.030

59. Y. Sasikumar, A.S. Adekunle, L.O. Olasunkanmi, I. Bahadur, R. Baskar, M.M. Kabanda, I.B. Obot and E.E. Ebenso, Experimental, quantum chemical and Monte Carlo simulation studies on the corrosion inhibition of some alkyl imidazolium ionic liquids containing tetrafluoroborate anion on mild steel in acidic medium, J. Mol. Liq., 2015, 211, 105-118. doi: 10.1016/j.molliq.2015.06.052

60. A.P. Hanza, E. Kowsari and M. Sayebani, Corrosion behavior of mild steel in $\mathrm{H}_{2} \mathrm{SO}_{4}$ solution with 1,4-di[1'-methylene-3'-methylimidazolium bromide]-benzene as an ionic liquid, Corros. Sci., 2016, 107, 96-106. doi: 10.1016/j.corsci.2016.02.023

61. Y. Guo, Y. Liu, W. Yang, X. Yin, Y. Chen, J. Le and Z. Chen, Corrosion inhibition properties of two imidazolium ionic liquids with hydrophilic tetrafluoroborate and hydrophobic hexafluorophosphate anions in acid medium, J. Ind. Eng. Chem., 2017, 56, 234-247. doi: $10.1016 /$ j.jiec.2017.07.016

62. M.A. Deyab, M.T. Zaky and M.I. Nessim, Inhibition of acid corrosion of carbon steel using four imidazoliumtetrafluoroborates ionic liquids, J. Mol. Liq., 2017, 229, 396404. doi: $10.1016 /$ j.molliq.2016.12.092

63. L. Feng, S. Zhang, Y. Qiang, S. Xu, B. Tan and S. Chen, The synergistic corrosion inhibition study of different chain lengths ionic liquids as green inhibitors for X70 steel in acidic medium, Mater. Chem. Phys., 2018, 215, 229-241. doi: 10.1016/j.matchemphys.2018.04.054

64. C. Verma, L.O. Olasunkanmi, I. Bahadur, H. Lgaz, M.A. Quraishi, J. Haque, ElS.M. Sherif and E.E. Ebenso, Experimental, density functional theory and molecular dynamics supported adsorption behavior of environmental benign imidazolium based ionic liquids on mild steel surface in acidic medium, J. Mol. Liq., 2019, 273, 1-15. doi: $\underline{10.1016 / j . m o l l i q .2018 .09 .139}$ 
65. E.K. Ardakani, E. Kowsari and A. Ehsani, Imidazolium-derived polymeric ionic liquid as a green inhibitor for corrosion inhibition of mild steel in $1.0 \mathrm{M} \mathrm{HCl}$ : Experimental and computational study, Colloids Surf., A, 2020, 586, 124195. doi: $\underline{10.1016 / j . c o l s u r f a .2019 .124195}$

66. N. Subasree and J.A. Selvi, Imidazolium based ionic liquid derivatives; synthesis and evaluation of inhibitory effect on mild steel corrosion in hydrochloric acid solution, Heliyon, 2020, 6, e03498. doi: 10.1016/j.heliyon.2020.e03498

67. A. Singh, K.R. Ansari, M.A. Quraishi and P. Banerjee, Corrosion inhibition and adsorption of imidazolium based ionic liquid over P110 steel surface in $15 \% \mathrm{HCl}$ under static and dynamic conditions: Experimental, surface and theoretical analysis, J. Mol. Liq., 2020, 323, 114608. doi: 10.1016/j.molliq.2020.114608

68. T. Yan, S. Zhang, L. Feng, Y. Qiang, L. Lu, D. Fu, Y. Wen, J. Chen, W. Li and B. Tan, Investigation of imidazole derivatives as corrosion inhibitors of copper in sulfuric acid: Combination of experimental and theoretical researches, J. Taiwan Inst. Chem. Eng., 2020, 106, 118-129. doi: $\underline{10.1016 / j . j t i c e .2019 .10 .014}$

69. Y. Qiang, S. Zhang, L. Guo, X. Zheng, B. Xiang and S. Chen, Experimental and theoretical studies of four allyl imidazoliumbased ionic liquids as green inhibitors for copper corrosion in sulfuric acid, Corros. Sci., 2017, 119, 68-78 doi: 10.1016/j.corsci.2017.02.021

70. R. Gasparac and E. Stupnisek-Lisac, Corrosion Protection on Copper by Imidazole and its Derivatives, Corrosion, 1999, 55, no. 11, 1031-1039. doi: $10.5006 / 1.3283940$

71. W.-J. Lee, Inhibiting effects of imidazole on copper corrosion in $1 \mathrm{M} \mathrm{HNO}_{3}$ solution, Mater. Sci. Eng. A, 2003, 348, 217-226. doi: 10.1016/S0921-5093(02)00734-7

72. M.N. El-Haddad and A.S. Fouda, Electroanalytical, quantum and surface characterization studies on imidazole derivatives as corrosion inhibitors for aluminum in acidic media, J. Mol. Liq., 2015, 209, 480-486. doi: 10.1016/j.molliq.2015.06.005

73. S. Ramkumar and D. Nalini, Enhanced corrosion resistance of mild steel in sulphuric acid medium by imidazole derivative: Experimental and computational studies, Mater. Today: Proc., 2019, 18, 1696-1708. doi: 10.1016/j.matpr.2019.05.266

74. M.M. Solomon, S.A. Umoren, M.A. Quraishi, D. Tripathi and E.J. Abai, Effect of alkyl chain length, flow, and temperature on the corrosion inhibition of carbon steel in a simulated acidizing environment by an imidazoline-based inhibitor, J. Pet. Sci. Eng., 2020, 187, 106801. doi: 10.1016/j.petrol.2019.106801

75. M.M. Solomon, S.A. Umoren, M.A. Quraishi and M. Salman, Myristic acid based imidazoline derivative as effective corrosion inhibitor for steel in $15 \% \mathrm{HCl}$ medium, $J$. Colloid Interface Sci., 2019, 551, 47-60. doi: 10.1016/j.jcis.2019.05.004

76. L. Zhang, Y. He, Y. Zhou, R. Yang, Q. Yang, D. Qing and Q. Niu, A novel imidazoline derivative as corrosion inhibitor for P110 carbon steel in hydrochloric acid environment, Petroleum, 2015, 1, 237-243. doi: 10.1016/j.petlm.2015.10.007 
77. J. Cruz, R. Martinez, J. Genesca and E. Garcia-Ochoa, Experimental and theoretical study of 1-(2-ethylamino)-2-methylimidazoline as an inhibitor of carbon steel corrosion in acid media, J. Electroanal. Chem., 2004, 566, 111-121. doi: 10.1016/i.jelechem.2003.11.018

78. K. Zhang, W. Yang, B. Xu, Y. Liu, X. Yin and Y. Chen, Corrosion inhibition of mild steel by bromide-substituted imidazoline in hydrochloric acid, J. Taiwan Inst. Chem. Eng., 2015, 57, 167-174. doi: 10.1016/j.jtice.2015.05.021

79. K. Zhang, B. Xu, W. Yang, X. Yin, Y. Liu and Y. Chen, Halogen-substituted imidazoline derivatives as corrosion inhibitors for mild steel in hydrochloric acid solution, Corros. Sci., 2014, 90, 284-295. doi: 10.1016/j.corsci.2014.10.032

80. A. Elbarki, W. Guerrab, T. Laabaissi, F. Benhiba, Z. Rouifi, H. Oudda, A. Guenbour, R. Touir, I. Warad, Y. Ramli and A. Zarrouk, Chemical, electrochemical and theoretical studies of 3-methyl-5,5'-diphenylimidazolidine-2,4-dione as corrosion inhibitor for mild steel in $\mathrm{HCl}$ solution, Chem. Data Collect., 2020, 28, 100454. doi: 10.1016/j.cdc.2020.100454

81. S.S. Abdel-Rehim, K.F. Khaled and N.A. Al-Mobarak, Corrosion inhibition of iron in hydrochloric acid using pyrazole, Arabian J. Chem., 2011, 4, 333-337. doi: 10.1016/j.arabjc.2010.06.056

82. N. Arrousse, R. Salim, Y. Kaddouri, A. Zarrouk, D. Zahri, F. El Hajjaji, R. Touzani, M. Taleb and S. Jodeh, The inhibition behavior of two pyrimidine-pyrazole derivatives against corrosion in hydrochloric solution: Experimental, surface analysis and in silico approach studies, Arabian J. Chem., 2020, 13, 5949-5965. doi: 10.1016/j.arabjc.2020.04.030

83. R.S. Abdel Hameed, H.I. Al-Shafey, A.S. Abul Magd and H.A. Shehata, Pyrazole Derivatives as Corrosion Inhibitor for C-Steel in Hydrochloric Acid Medium, J. Mater. Environ. Sci., 2012, 3, no. 2, 294-305.

84. K. Cherrak, M.E. Belghiti, A. Berrissoul, M. El Massaoudi, M. El Faydy, M. Taleb, S. Radi, A. Zarrouk and A. Dafali, Pyrazole carbohydrazide as corrosion inhibitor for mild steel in $\mathrm{HCl}$ medium: Experimental and theoretical investigations, Surf. Interfaces, 2020, 20, 100578. doi: $10.1016 /$ j.surfin.2020.100578

85. S. El Arrouji， K. Ismaily Alaoui, A. Zerrouki, S. El Kadiri, R. Touzani, Z. Rais, M. Filali Baba, M. Taleb, A. Chetouani and A. Aouniti, The Influence of Some Pyrazole Derivatives on The Corrosion Behaviour of Mild Steel in 1M HCl Solution, J. Mater. Environ. Sci., 2016, 7, no. 1, 299-309.

86. S. El Arrouji, K. Karrouchi, A. Berisha, K.I. Alaoui, I. Warad, Z. Rais, S. Radi, M. Taleb, M. Ansar and A. Zarrouk, New pyrazole derivatives as effective corrosion inhibitors on steelelectrolyte interface in $1 \mathrm{M} \mathrm{HCl}$ : Electrochemical, surface morphological (SEM) and computational analysis, Colloids Surf., A, 2020, 604, 125325. doi: $\underline{10.1016 / j . c o l s u r f a .2020 .125325}$ 
87. F. Boudjellal, H.B. Ouici, A. Guendouzi, O. Benali and A. Sehmi, Experimental and theoretical approach to the corrosion inhibition of mild steel in acid medium by a newly synthesized pyrazole carbothioamide heterocycle, J. Mol. Struct., 2020, 1199, 127051. doi: $10.1016 /$ j.molstruc.2019.127051

88. M. Bouklah, A. Attayibat, B. Hammouti, A. Ramdani, S. Radi and M. Benkaddour, Pyridine-pyrazole compound as inhibitor for steel in $1 \mathrm{M} \mathrm{HCl}$, Appl. Surf. Sci., 2005, 240, 341-348. doi: 10.1016/j.apsusc.2004.07.001

89. P.K. Paul, M. Yadav and I.B. Obot, Investigation on corrosion protection behavior and adsorption of carbohydrazide-pyrazole compounds on mild steel in $15 \% \mathrm{HCl}$ solution: Electrochemical and computational approach, J. Mol. Liq., 2020, 314, 113513. doi: 10.1016/j.molliq.2020.113513

90. L. Herrag, A. Chetouani, S. Elkadiri, B. Hammouti and A. Aouniti, Pyrazole Derivatives as Corrosion Inhibitors for Steel in Hydrochloric Acid, Port. Electrochim. Acta, 2008, 26, 211-220. doi: 10.4152/pea.200802211

91. Y.E. Louadi, F. Abrigach, A. Bouyanzer, R. Touzani, A. El Assyry, A. Zarrouk and B. Hammouti, Theoretical and Experimental Studies on the Corrosion Inhibition Potentials of Two Tetrakis Pyrazole Derivatives for Mild Steel in $1.0 \mathrm{M} \mathrm{HCl}$, Port. Electrochim. Acta, 2017, 35, no. 3, 159-178. doi: 10.4152/pea.201703159

92. C. Verma, V.S. Saji, M.A. Quraishi and E.E. Ebenso, Pyrazole derivatives as environmental benign acid corrosion inhibitors for mild steel: Experimental and computational studies, J. Mol. Liq., 2019, 298, 111943. doi: 10.1016/j.molliq.2019.111943

93. M. Abdallah and M.M. El-Naggar, $\mathrm{Cu}^{+2}$ cation $+3,5$-dimethyl pyrazole mixture as a corrosion inhibitor for carbon steel in sulfuric acid solution, Mater. Chem. Phys., 2001, 71, 291-298. doi: 10.1016/S0254-0584(01)00289-9

94. A.G. Gad Allah, M.M. Hefny, S.A. Salih and M.S. EI-Basiouny, Corrosion Inhibition of Zinc in HCI Solution by Several Pyrazole Derivatives, Corrosion, 1989, 45, no. 7, 574578. doi: $10.5006 / 1.3577875$

95. H.S. Gadow, T.A. Farghaly and A.M. Eldesoky, Experimental and theoretical investigations for some spiropyrazoles derivatives as corrosion inhibitors for copper in $2 \mathrm{M} \mathrm{HNO}_{3}$ solutions, J. Mol. Liq., 2019, 294, 111614. doi: 10.1016/j.molliq.2019.111614

96. N. Anusuya, P. Sounthari, J. Saranya, K. Parameswari and S. Chitra, Corrosion inhibition effect of hydroxy pyrazoline derivatives on mild steel in sulphuric acid solution together with Quantum chemical studies, J. Mater. Environ. Sci., 2015, 6, no. 6, $1606-1623$.

97. H. Lgaz, R. Salghi, A. Chaouiki, Shubhalaxmi, S. Jodeh and K. Subrahmanya Bhat, Pyrazoline derivatives as possible corrosion inhibitors for mild steel in acidic media: A combined experimental and theoretical approach, Cogent Eng., 2018, 5, 1441585. doi: $\underline{10.1080 / 23311916.2018 .1441585}$ 
98. H. Lgaz, S.Kr. Saha, A. Chaouiki, K. Subrahmanya Bhat, R. Salghi, Shubhalaxmi, P. Banerjee, I.H. Ali, M.I. Khan and I.-M. Chung, Exploring the potential role of pyrazoline derivatives in corrosion inhibition of mild steel in hydrochloric acid solution: Insights from experimental and computational studies, Constr. Build. Mater., 2020, 233, 117320. doi: 10.1016/j.conbuildmat.2019.117320

99. A. Sehmi, H.B. Ouici, A. Guendouzi, M. Ferhat, O. Benali and F. Boudjellal, Corrosion Inhibition of Mild Steel by newly Synthesized Pyrazole Carboxamide Derivatives in $\mathrm{HCl}$ Acid Medium: Experimental and Theoretical Studies, J. Electrochem. Soc., 2020, 167, 155508.

100. I. Selatnia, A. Sid, M. Benahmed, O. Dammene debbih, T. Ozturk and N. Gherraf, Synthesis and Characterization of a Bis-Pyrazoline Derivative as Corrosion Inhibitor for A283 Carbon Steel in 1M HCl: Electrochemical, Surface, DFT and MD Simulation Studies, Prot. Met. Phys. Chem. Surf., 2018, 54, 1182-1193. doi: 10.1134/S2070205118060229

101. N.P. Swathi, V.D.P. Alva and S. Samshuddin, A Review on 1,2,4-Triazole Derivatives as Corrosion Inhibitors, J. Bio Tribo Corros., 2017, 3, 42. doi: 10.1007/s40735-017$\underline{0102-3}$

102. M. Faisal, A. Saeed, D. Shahzad, N. Abbas, F.A. Larik, P.A. Channar, T.A. Fattah, D.M. Khan and S.A. Shehzadi, General properties and comparison of the corrosion inhibition efficiencies of the triazole derivatives for mild steel, Corros. Rev., 2018, 36, no. 6, 507-545. doi: 10.1515/corrrev-2018-0006

103. A. Boutouil, M.R. Laamari, I. Elazhary, L. Bahsis, H. Anane and S.-E. Stiriba, Towards a deeper understanding of the inhibition mechanism of a new 1,2,3-triazole derivative for mild steel corrosion in the hydrochloric acid solution using coupled experimental and theoretical methods, Mater. Chem. Phys., 2020, 241, 122420. doi: 10.1016/j.matchemphys.2019.122420

104. G.O. Resende, S.F. Teixeira, I.F. Figueiredo, A.A. Godoy, D.J.F. Lougon, B.A. Cotrim and F.C. de Souza, Synthesis of 1,2,3-Triazole Derivatives and Its Evaluation as Corrosion Inhibitors for Carbon Steel, Int. J. Electrochem., 2019, 2019, 6759478. doi: $10.1155 / 2019 / 6759478$

105. Q. Deng, N.-N. Ding, X.-L. Wei, L. Cai, X.-P. He, Y.-T. Long, G.-R. Chen and $\mathrm{K}$. Chen, Identification of diverse 1,2,3-triazole-connected benzyl glycosideserine/threonine conjugates as potent corrosion inhibitors for mild steel in $\mathrm{HCl}$, Corros. Sci., 2012, 64, 64-73. doi: 10.1016/j.corsci.2012.07.001

106. C.M. Fernandes, L.X. Alvarez, N.E. dos Santos, A.C. Maldonado Barrios and E.A. Ponzio, Green synthesis of 1-benzyl-4-phenyl-1H-1,2,3-triazole, its application as corrosion inhibitor for mild steel in acidic medium and new approach of classical electrochemical analyses, Corros. Sci., 2019, 149, 185-194. doi: $\underline{10.1016 / j . c o r s c i .2019 .01 .019}$ 
107. Q. Ma, S. Qi, X. He, Y. Tang and G. Lu, 1,2,3-Triazole derivatives as corrosion inhibitors for mild steel in acidic medium: Experimental and computational chemistry studies, Corros. Sci., 2017, 129, 91-101. doi: 10.1016/j.corsci.2017.09.025

108. Z. Rouifi, F. Benhiba, M. El Faydy, T. Laabaissi, H. About, H. Oudda, I. Warad, A. Guenbour, B. Lakhrissi and A. Zarrouk, Performance and computational studies of new soluble triazole as corrosion inhibitor for carbon steel in $\mathrm{HCl}$, Chem. Data Collect., 2019, 22, 100242. doi: $10.1016 /$ j.cdc.2019.100242

109. Q.A. Jawad, A.Q. Hameed, M.K. Abood, A.A. Al-Amiery, L.M. Shaker, A.A.H. Kadhum and M.S. Takriff, Synthesis and comparative study of novel triazole derived as corrosion inhibitor of mild steel in $\mathrm{HCl}$ medium complemented with DFT calculations, Int. J. Corros. Scale Inhib., 2020, 9, no. 2, 688-705. doi: 10.17675/23056894-2020-9-2-19

110. H. Rahmani, K.I. Alaoui, M. El Azzouzi, F. Benhiba, A. El Hallaoui, Z. Rais, M. Taleb, A. Saady, B. Labriti, A. Aouniti and A. Zarrouk, Corrosion assessement of mild steel in acid environment using novel triazole derivative as a anti-corrosion agent: A combined experimental and quantum chemical study, Chem. Data Collect., 2019, 24, 100302. doi: 10.1016/j.cdc.2019.100302

111. F. Bentiss, C. Jama, B. Mernari, H. El Attari, L. El Kadi, M. Lebrini, M. Traisnel and M. Lagrenee, Corrosion control of mild steel using 3,5-bis(4-methoxyphenyl)-4-amino1,2,4-triazole in normal hydrochloric acid medium, Corros. Sci., 2009, 51, 1628-1635. doi: $10.1016 /$ j.corsci.2009.04.009

112. B. El Mehdi, B. Mernari, M. Traisnel, F. Bentiss and M. Lagrenée, Synthesis and comparative study of the inhibitive effect of some new triazole derivatives towards corrosion of mild steel in hydrochloric acid solution, Mater. Chem. Phys., 2002, 77, 489496. doi: $10.1016 / \mathrm{S} 0254-0584(02) 00085-8$

113. S. John and A. Joseph, Electro analytical, surface morphological and theoretical studies on the corrosion inhibition behavior of different 1,2,4-triazole precursors on mild steel in $1 \mathrm{M}$ hydrochloric acid, Mater. Chem. Phys., 2012, 133, 1083-1091. doi: 10.1016/j.matchemphys.2012.02.020

114. M. Tourabi, K. Nohair, M. Traisnel, C. Jama and F. Bentiss, Electrochemical and XPS studies of the corrosion inhibition of carbon steel in hydrochloric acid pickling solutions by 3,5-bis(2-thienylmethyl)-4-amino-1,2,4-triazole, Corros. Sci., 2013, 75, 123-133. doi: $10.1016 /$ j.corsci.2013.05.023

115. L. Wang, Inhibition of mild steel corrosion in phosphoric acid solution by triazole derivatives, Corros. Sci., 2006, 48, 608-616. doi: 0.1016/j.corsci.2005.02.007

116. M. El Belghiti, Y. Karzazi, A. Dafali, B. Hammouti, F. Bentiss, I.B. Obot, I. Bahadur and E.E. Ebenso, Experimental, quantum chemical and Monte Carlo simulation studies of 3,5-disubstituted-4-amino-1,2,4-triazoles as corrosion inhibitors on mild steel in acidic medium, J. Mol. Liq., 2016, 218, 281-293. doi: 10.1016/j.molliq.2016.01.076 
117. M.E. Belghiti, Y. Karzazi, A. Dafali, I.B. Obot, E.E. Ebenso, K.M. Emran, I. Bahadur, B. Hammouti and F. Bentiss, Anti-corrosive properties of 4-amino-3,5bis(disubstituted)-1,2,4-triazole derivatives on mild steel corrosion in $2 \mathrm{M} \mathrm{H}_{3} \mathrm{PO}_{4}$ solution: Experimental and theoretical studies, J. Mol. Liq., 2016, 216, 874-886. doi: 10.1016/j.molliq.2015.12.093

118. P.R. Ammal, M. Prajila and A. Joseph, Physicochemical studies on the inhibitive properties of a 1,2,4-triazole Schiff's base, HMATD, on the corrosion of mild steel in hydrochloric acid, Egypt. J. Pet., 2018, 27, 307-317. doi: 10.1016/j.ejpe.2017.05.002

119. K.R. Ansari, M.A. Quraishi and A. Singh, Schiff's base of pyridyl substituted triazoles as new and effective corrosion inhibitors for mild steel in hydrochloric acid solution, Corros. Sci., 2014, 79, 5-15. doi: 10.1016/j.corsci.2013.10.009

120. T.K. Chaitra, K.N.S. Mohana and H.C. Tandon, Thermodynamic, electrochemical and quantum chemical evaluation of some triazole Schiff bases as mild steel corrosion inhibitors in acid media, J. Mol. Liq., 2015, 211, 1026-1038. doi: 10.1016/j.molliq.2015.08.031

121. H.-L. Wang, H.-B. Fan and J.-S. Zheng, Corrosion inhibition of mild steel in hydrochloric acid solution by a mercapto-triazole compound, Mater. Chem. Phys., 2002, 77, 655-661. doi: 10.1016/S0254-0584(02)00123-2

122. H.-L. Wang, R.-B. Liu and J. Xin, Inhibiting effects of some mercapto-triazole derivatives on the corrosion of mild steel in $1.0 \mathrm{M} \mathrm{HCl}$ medium, Corros. Sci., 2004, 46, 2455-2466. doi: $10.1016 /$ j.corsci.2004.01.023

123. M. Murmu, S.K. Saha, P. Bhaumick, N.C. Murmu, H. Hirani and P. Banerjee, Corrosion inhibitio nproperty of azomethine functionalized triazole derivatives in $1 \mathrm{molL}^{-1} \mathrm{HCl}$ medium for mild steel: Experimental and theoretical exploration, J. Mol. Liq., 2020, 313, 113508. doi: 10.1016/j.molliq.2020.113508

124. Ya.G. Avdeev, Protection of Steel in Solutions of Mineral Acids Using $\alpha, \beta$-Unsaturated Aldehydes, Ketones, and Azomethines, Prot. Met. Phys. Chem. Surf., 2015, 51, no. 7, 1140-1148. doi: $10.1134 /$ S2070205115070023

125. Ya.G. Avdeev, A.Yu. Luchkin and M.V. Tyurina, Osobennosti zashchity nizkouglerodistoi stali $\mathrm{v}$ rastvorakh mineral'nykh kislot $\mathrm{N}$-tsinnamiliden-1N-1,2,4triazol-3-aminom (Specifics of protection of low-carbon steel in solutions of mineral acids by N-cinnamylidene-1H-1,2,4-triazole-3-amine), Korroz.: Mater., Zashch. (Corros.: Mater., Prot.), 2014, no. 8, 38-40 (in Russian).

126. Ya.G. Avdeev and Yu.I. Kuznetsov, Inhibitory protection of steels from hightemperature corrosion in acid solutions. A review. Part 1., Int. J. Corros. Scale Inhib., 2020, 9, no. 2, 394-426. doi: 10.17675/2305-6894-2020-9-2-2

127. Ya.G. Avdeev, Yu.I. Kuznetsov and A.K. Buryak, Inhibition of steel corrosion by unsaturated aldehydes in solutions of mineral acids, Corros. Sci., 2013, 69, 50-60. doi: $\underline{10.1016 / j . c o r s c i .2012 .11 .016}$ 
128. L.-G. Qiu, A.-J. Xie and Y.-H. Shen, A novel triazole-based cationic gemini surfactant: synthesis and effect on corrosion inhibition of carbon steel in hydrochloric acid, Mater. Chem. Phys., 2005, 91, 269-273. doi: 10.1016/j.matchemphys.2004.11.022

129. M. Naciri, Y. El Aoufir, H. Lgaz, F. Lazrak, A. Ghanimi, A. Guenbour, I.H. Ali, M. El Moudane, J.l. Taoufik and I.-M. Chung, Exploring the potential of a new 1,2,4triazole derivative for corrosion protection of carbon steel in $\mathrm{HCl}$ : A computational and experimental evaluation, Colloids Surf., A, 2020, 597, 124604. doi: 10.1016/j.colsurfa.2020.124604

130. Y. El Aoufir, R. Aslam, F. Lazrak, R. Marzouki, S. Kaya, S. Skal, A. Ghanimi, I.H. Ali, A. Guenbour, H. Lgaz and I.-M. Chung, The effect of the alkyl chain length on corrosion inhibition performances of 1,2,4-triazole-based compounds for mild steel in $1.0 \mathrm{M} \mathrm{HCl}$ : Insights from experimental and theoretical studies, J. Mol. Liq., 2020, 303, 112631. doi: 10.1016/j.molliq.2020.112631

131. Ya.G. Avdeev, P.A. Belinskii, Yu.I. Kuznetsov and O.O. Zel', High-Temperature Inhibitor of Steel Corrosion in Sulfuric Acid Solution, Prot. Met. Phys. Chem. Surf., 2010, 46, no. 7, 782-787. doi: 10.1134/S2070205110070087

132. Ya.G. Avdeev, A.Yu. Luchkin, Yu.I. Kuznetsov, I.G. Gorichev and M.V. Tyurina, Zashchita nizkouglerodistoi stali $\mathrm{v}$ solyanokislykh rastvorakh $\mathrm{v}$ usloviyakh vysokotemperaturnoi korrozii (do $160^{\circ} \mathrm{C}$ ) (Protection of low carbon steel in hydrochloric acid solutions from high temperature corrosion (up to $160^{\circ} \mathrm{C}$ ), Korroz.: Mater., Zashch. (Corros.: Mater., Prot.), 2011, no. 10, 26-31 (in Russian).

133. Ya.G. Avdeev, A.Yu. Luchkin, Yu.I. Kuznetsov, I.G. Gorichev and M.V. Tyurina, Zashchita nizkouglerodistoi stali $\mathrm{v}$ serno-kislykh rastvorakh ot vysokotemperaturnoi korrozii (do $200^{\circ} \mathrm{C}$ ) (Protection of low carbon steel in sulfuric acid solutions from high temperature corrosion (up to $\left.200^{\circ} \mathrm{C}\right)$ ), Korroz.: mater., zashch. (Corros.: Mater., Prot.), 2011, no. 8, 20-26 (in Russian).

134. Ya.G. Avdeev, Yu.I. Kuznetsov and O.O. Zel', Novyi ingibitor kislotnoi korrozii stali IFKhAN-93 (New steel acid corrosion inhibitor - IFKhAN-93), Korroz.: Mater., Zashch. (Corros.: Mater., Prot.), 2010, no. 7, 12-17 (in Russian).

135. Ya.G. Avdeev, Yu.I. Kuznetsov and O.O Zel', Zashchita ot korrozii nizkouglerodistoi stali v rastvorakh mineral'nykh kislot ingibitorom IFKhAN-94 (Corrosion protection of low-carbon steel in solutions of mineral acids by IFKhAN-94 inhibitor), Praktika protivokorrozionnoy zashchity (Practice of Corrosion Protection), 2011, no. 1, 8-13 (in Russian).

136. M.V. Tyurina, Ya.G. Avdeev, L.P. Kazanskii, A.Yu. Luchkin and O.A. Kireeva, Protection of Low-Carbon Steel from Corrosion in Hydrochloric Acid by 4-Substituted 1,2,4-Triazole, Prot. Met. Phys. Chem. Surf., 2016, 52, no. 7, 1147-1152. doi: 10.1134/S2070205116070157

137. Ya.G. Avdeev, I.G. Gorichev and A.Yu. Luchkin, Effect of IFKhAN-92 inhibitor on scale removal during sulfuric acid pickling of steel, Int. J. Corros. Scale Inhib., 2012, 1, no. 1, 26-37. doi: $10.17675 / 2305-6894-2012-1-1-026-037$ 
138. Ya.G. Avdeev, L.V. Frolova, Yu.I. Kuznetsov and O.O. Zel', Vliyanie proizvodnykh triazola na korroziyu i navodorozhivanie vysokoprochnoi stali v rastvorakh mineral'nykh kislot (Effect of Triazole Derivatives on Corrosion and Hydrogenation of High-Strength Steel in Mineral Acid Solutions), Korroz.: Mater., Zashch. (Corros.: Mater., Prot.), 2010, no. 5, 22-29 (in Russian).

139. N. Shet, R. Nazareth and P.A. Suchetan, Corrosion inhibition of 316 stainless steel in $2 \mathrm{M} \mathrm{HCl}$ by $4-\{[4-($ dimethylamino)benzylidene]amino $\}-5$-methyl-4H-1,2,4- triazole-3thiol, Chem. Data Collect., 2019, 20, 100209. doi: 10.1016/j.cdc.2019.100209

140. Ya.G. Avdeev, D.S. Kuznetsov, M.V. Tyurina, A.Yu. Luchkin and M.A. Chekulaev, Protection of chromium-nickel steel in hydrochloric acid solution by a substituted triazole, Int. J. Corros. Scale Inhib., 2015, 4, no. 1, 1-14. doi: 10.17675/2305-68942015-4-1-001-014

141. Ya.G. Avdeev, L.V. Frolova, D.S. Kuznetsov, M.V. Tyurina and M.A. Chekulaev, Protection of stainless steel in hydrochloric acid solution containing hydrogen sulfide by inhibitors, Int. J. Corros. Scale Inhib., 2016, 5, no. 1, 87-99. doi: 10.17675/2305-68942016-5-1-7

142. Ya.G. Avdeev, D.S. Kuznetsov, M.V. Tyurina, S.V. Oleynik and M.A. Chekulaev, High-temperature inhibitors of stainless steel corrosion in hydrochloric acid solutions, Int. J. Corros. Scale Inhib., 2017, 6, no. 2, 180-195. doi: 10.17675/2305-6894-2017-6$\frac{2-7}{Y a}$

143. Ya.G. Avdeev, D.S. Kuznetsov, M.V. Tyurina and M.A. Chekulaev, Protection of nickel-chromium steel in sulfuric acid solution by a substituted triazole, Int. J. Corros. Scale Inhib., 2015, 4, no. 2, 146-161. doi: 10.17675/2305-6894-2015-4-1-146-161

144. Ya.G. Avdeev, L.V. Frolova, D.S. Kuznetsov, M.V. Tyurina and M.A. Chekulaev, Protection of stainless steel in sulfuric acid solution containing hydrogen sulfide by inhibitors, Int. J. Corros. Scale Inhib., 2016, 5, no. 2, 147-158. doi: 10.17675/23056894-2016-5-2-4

145. Sudheer and M.A. Quraishi, Electrochemical and theoretical investigation of triazole derivatives on corrosion inhibition behavior of copper in hydrochloric acid medium, Corros. Sci., 2013, 70, 161-169. doi: 10.1016/j.corsci.2013.01.025

146. L.J. Berchmans, V. Sivan and S.V.K. Iyer, Studies on triazole derivatives as inhibitors for the corrosion of muntz metal in acidic and neutral solutions, Mater. Chem. Phys., 2006, 98, 395-400. doi: 10.1016/j.matchemphys.2005.09.053

147. M.E. Belghiti, Y. El Oudadi, S. Echihi, A. Elmelouky, H. Outada, Y. Karzazi, M. Bakasse, C. Jama, F. Bentiss and A. Dafali, Anticorrosive properties of two 3,5dissubstitud-4-amino-1,2,4-triazole derivatives on copper in hydrochloric acid environment: Ac impedance, thermodynamic and computational investigations, Surf. Interfaces, 2020, 21, 100692. doi: 10.1016/j.surfin.2020.100692 
148. Ya.G. Avdeev and Yu.I. Kuznetsov, Zashchita tsvetnykh metallov v rastvorakh mineral'nykh kislot ingibitorom IFKhAN-92 (Protection of non-ferrous metals in solutions of mineral acids by inhibitor IFKhAN-92), Korroz.: Mater., Zashch. (Corros.: Mater., Prot.), 2011, no. 5, 30-33 (in Russian).

149. S. Kertit and B. Hammouti, Corrosion inhibition of iron in $1 \mathrm{M} \mathrm{HCl}$ by 1 -phenyl-5mercapto-1,2,3,4-tetrazole, Appl. Surf. Sci., 1996, 93, no. 1, 59-66. doi: 10.1016/01694332(95)00189-1

150. P. Xuehui, H. Baorong, L. Weihua, L. Faqian and Y. Zhigang, 2,3,5-Triphenyl- $2 H$ tetrazolium Chloride and 2,4,6-Tri(2-pyridyl)-s-triazine on the Corrosion of Mild Steel in HCI, Chin. J. Chem. Eng., 2007, 15, no. 6, 909-915. doi: 10.1016/S10049541(08)60024-5

151. X. Li, S. Deng and H. Fu, Blue tetrazolium as a novel corrosion inhibitor for cold rolled steel in hydrochloric acid solution, Corros. Sci., 2010, 52, 2786-2792. doi: 10.1016/j.corsci.2010.04.020

152. C. Verma, M.A. Quraishi and A. Singh, 5-Substituted 1H-tetrazoles as effective corrosion inhibitors for mildsteel in $1 \mathrm{M}$ hydrochloric acid, J. Taibah Univ. Sci., 2016, 10, 718-733. doi: 10.1016/j.jtusci.2015.10.005

153. A.M. Eid, S. Shaaban and K. Shalabi, Tetrazole-based organoselenium bifunctionalized corrosion inhibitors during oil well acidizing: Experimental, computational studies, and SRB bioassay, J. Mol. Liq., 2020, 298, 111980. doi: 10.1016/j.molliq.2019.111980

154. B.G. Ateya, B.M.A. El-Khair and R. Al-Qasimi, Inhibition of the acid corrosion of iron with triphenyl tetrazolium chloride, Corros. Sci., 1982, 22, no. 8, 717-721. doi: 10.1016/0010-938X(82)90009-9

155. X. Li, S. Den and H. Fu, Synergism between red tetrazolium and uracil on the corrosion of cold rolled steel in $\mathrm{H}_{2} \mathrm{SO}_{4}$ solution, Corros. Sci., 2009, 51, 1344-1355. doi: 10.1016/j.corsci.2009.03.023

156. X. Li, S. Den and H. Fu, Nitrotetrazolium blue chloride as a novel corrosion inhibitor of steel in sulfuric acid solution, Corros. Sci., 2010, 52, 3840-3846. doi: 10.1016/j.corsci.2010.07.020

157. X. Li, S. Den and H. Fu, Triazolyl blue tetrazolium bromide as a novel corrosion inhibitor for steel in $\mathrm{HCl}$ and $\mathrm{H}_{2} \mathrm{SO}_{4}$ solutions, Corros. Sci., 2011, 53, 302-309. doi: 10.1016/j.corsci.2010.09.036

158. X. Li, S. Den and H. Fu, Blue tetrazolium as a novel corrosion inhibitor for cold rolled steel in sulfuric acid solution, Mater. Chem. Phys., 2011, 129, 696-700. doi: 10.1016/j.matchemphys.2011.05.042

159. X. Li, S. Den and H. Fu, Red tetrazolium as an effective inhibitor for the corrosion of cold rolled steel in $7.0 \mathrm{~mol} \cdot \mathrm{L}^{-1} \mathrm{H}_{2} \mathrm{SO}_{4}$ solution, Chin. J. Chem. Eng., 2018, 26, no. 12, 2641-2653. doi: 10.1016/j.cjche.2018.02.029 
160. H. Ma, T. Song, H. Sun and X. Li, Experimental and theoretical elucidation on the inhibition mechanism of 1-methyl-5-mercapto-1,2,3,4-tetrazole self-assembled films on corrosion of iron in $0.5 \mathrm{M} \mathrm{H}_{2} \mathrm{SO}_{4}$ Solutions, Thin Solid Films, 2008, 516, 1020-1024. doi: $10.1016 /$ j.tsf.2007.06.225

161. B. Tan, S. Zhang, J. He, W. Li, Y. Qiang, Q. Wan, C. Xu and S. Chen, Insight into anticorrosion mechanism of tetrazole derivatives for X80 steel in $0.5 \mathrm{M} \mathrm{H}_{2} \mathrm{SO}_{4}$ medium: Combined experimental and theoretical researches, J. Mol. Liq., 2020, 114464. doi: 10.1016/j.molliq.2020.114464

162. Ya.G. Avdeev, Yu.I. Kuznetsov, V.A. Ostrovskiy, M.V. Tyurina, P.A. Aleshunin and O.O. Zel', Zashchita nizkouglerodistoi stali $\mathrm{v}$ rastvorakh mineral'nykh kislot proizvodnymi tetrazola (Protection of low-carbon steel in mineral acid solutions by tetrazole derivatives), Korroz.: Mater., Zashch. (Corros.: Mater., Prot.), 2011, no. 4, 28-32 (in Russian).

163. S. Deng, X. Li and G. Du, Two ditetrazole derivatives as effective inhibitors for the corrosion of steel in $\mathrm{CH}_{3} \mathrm{COOH}$ solution, J. Mater. Res. Technol., 2019, 8, no. 1, 13891399. doi: $\underline{10.1016 / j . j m r t .2018 .09 .010}$

164. X. Li, S. Deng, T. Lin, X. Xie and X. Xu, Inhibition action of triazolyl blue tetrazolium bromide on cold rolled steel corrosion in three chlorinated acetic acids, J. Mol. Liq., 2019, 274, 77-89. doi: 10.1016/j.molliq.2018.10.066

165. M.S. El-Sayed, R.M. Erasmus and J.D. Comins, Inhibition of copper corrosion in acidic chloride pickling solutions by 5-(3-aminophenyl)-tetrazole as a corrosion inhibitor, Corros. Sci., 2008, 50, 3439-3445. doi: 10.1016/j.corsci.2008.10.002

166. A.H. El-Askalany, S.I. Mostafa, K. Shalabi, A.M. Eid and S. Shaaban, Novel tetrazolebased symmetrical diselenides as corrosion inhibitors for N80 carbon steel in $1 \mathrm{M} \mathrm{HCl}$ solutions: Experimental and theoretical studies, J. Mol. Liq., 2016, 223, 497-508. doi: 10.1016/j.molliq.2016.08.088

167. B. Tan, S. Zhang, H. Liu, Y. Qiang, W. Li, L. Guo and S. Chen, Insights into the inhibition mechanism of three 5-phenyltetrazole derivatives for copper corrosion in sulfuric acid medium via experimental and DFT methods, J. Taiwan Inst. Chem. Eng., 2019, 102, 424-437. doi: 10.1016/j.jtice.2019.06.005

168. Y. Qiang, H. Li and X. Lan, Self-assembling anchored film basing on two tetrazole derivatives for application to protect copper in sulfuric acid environment, J. Mater. Sci. Technol., 2020, 52, 63-71. doi: 10.1016/j.jmst.2020.04.005

169. M. Mihit, K. Laarej, H. Abou El Makarim, L. Bazzi, R. Salghi and B. Hammouti, Study of the inhibition of the corrosion of copper and zinc in $\mathrm{HNO}_{3}$ solution by electrochemical technique and quantum chemical calculations, Arabian J. Chem., 2010, 3, 55-60. doi: 10.1016/j.arabjc.2009.12.009

170. K.F. Khaled and M.M. Al-Qahtani, The inhibitive effect of some tetrazole derivatives towards Al corrosion in acid solution: Chemical, electrochemical and theoretical studies, Mater. Chem. Phys., 2009, 113, 150-158. doi: 10.1016/j.matchemphys.2008.07.060 
171. T.M. Lv, S.H. Zhu, L. Guo and S.T. Zhang, Experimental and theoretical investigation of indole as a corrosion inhibitor for mild steel in sulfuric acid solution, Res. Chem. Intermed., 2015, 41, 7073-7093. doi: 10.1007/s11164-014-1799-y

172. G. Moretti, G. Quartarone, A. Tassan and A. Zlngales, 5-Amino- and 5-chloro-indole as mild steel corrosion inhibitors in $1 \mathrm{~N}$ sulphuric acid, Electrochim. Acta, 1996, 41, no. 13, 1971-1980. doi: 10.1016/0013-4686(95)00485-8

173. K.F. Khaled, Application of electrochemical frequency modulation for monitoring corrosion and corrosion inhibition of iron by some indole derivatives in molar hydrochloric acid, Mater. Chem. Phys., 2008, 112, 290-300. doi: 10.1016/j.matchemphys.2008.05.056

174. C. Verma, M.A. Quraishi, E.E. Ebenso, I.B. Obot and A. El Assyry, 3-Amino alkylated indoles as corrosion inhibitors for mild steel in $1 \mathrm{M} \mathrm{HCl}$ : Experimental and theoretical studies, J. Mol. Liq., 2016, 219, 647-660. doi: 10.1016/j.molliq.2016.04.024

175. P. Kumari, P. Shetty and S.A. Rao, Corrosion Inhibition Effect of 4-Hydroxy-N'-[(E)( $1 H$-indole-2-ylmethylidene)]Benzohydrazide on Mild Steel in Hydrochloric Acid Solution, Int. J. Corros., 2014, 2014, 256424. doi: 10.1155/2014/256424

176. S.A. Ahmed, M.I. Awad, I.I. Althagafi, H.M. Altass, M. Morad, A. Alharbi and R.J. Obaid, Newly synthesized indolium-based ionic liquids as unprecedented inhibitors for the corrosion of mild steel in acid medium, J. Mol. Liq., 2019, 291, 111356. doi: $\underline{10.1016 / j . m o l l i q .2019 .111356}$

177. G. Avci, Corrosion inhibition of indole-3-acetic acid on mild steel in $0.5 \mathrm{M} \mathrm{HCl}$, Colloids Surf. A, 2008, 317, 730-736. doi: 10.1016/j.colsurfa.2007.12.009

178. G. Quartarone, L. Bonaldo and C. Tortato, Inhibitive action of indole-5-carboxylic acid towards corrosion of mild steel in deaerated $0.5 \mathrm{M}$ sulfuric acid solutions, Appl. Surf. Sci., 2006, 252, 8251-8257. doi: 10.1016/j.apsusc.2005.10.051

179. G. Quartarone, M. Battilana, L. Bonaldo and T. Tortato, Investigation of the inhibition effect of indole-3-carboxylic acid on the copper corrosion in $0.5 \mathrm{M} \mathrm{H}_{2} \mathrm{SO}_{4}$, Corros. Sci., 2008, 50, 3467-3474. doi: 10.1016/j.corsci.2008.09.032

180. H.U. Nwankwo, E.D. Akpan, L.O. Olasunkanmi, C. Verma, A.M. Al-Mohaimeed, D.A. Al Farraj and E.E. Ebenso, N-substituted carbazoles as corrosion inhibitors in microbiologically influenced and acidic corrosion of mild steel: Gravimetric, electrochemical, surface and computational studies, J. Mol. Struct., 2021, 1223, 129328. doi: $10.1016 /$ j.molstruc.2020.129328

181. H.U. Nwankwo, L.O. Olasunkanmi and E.E. Ebenso, Electrochemical and Computational Studies of Some Carbazole Derivatives as Inhibitors of Mild Steel Corrosion in Abiotic and Biotic Environments, J. Bio Tribo Corros., 2018, 4, 13. doi: 10.1007/s40735-018-0130-7

182. M. Lebrini, F. Robert, H. Vezin and C. Roos, Electrochemical and quantum chemical studies of some indole derivatives as corrosion inhibitors for C38 steel in molar hydrochloric acid, Corros. Sci., 2010, 52, 3367-3376. doi: 10.1016/j.corsci.2010.06.009 
183. F. Bentiss, F. Gassama, D. Barbry, L. Gengembre, H. Vezin, M. Lagrenee and M. Traisnel, Enhanced corrosion resistance of mild steel in molar hydrochloric acid solution by 1,4-bis(2-pyridyl)-5H-pyridazino[4,5-b]indole: Electrochemical, theoretical and XPS studies, Appl. Surf. Sci., 2006, 252, 2684-2691. doi: 10.1016/j.apsusc.2005.03.231

184. Y. Wang, Z. Yang, R. Wang, W. Chen, M. Ding, P.R. China, F. Zhan and B. Hou, Highefficiency Corrosion Inhibitor for Acidizing: Synthesis, Characterization and Anticorrosion Performance of Novel Indolizine Derivative, Soc. Pet. Eng., SPE International Conference on Oilfield Chemistry, 8-9 April, Galveston, Texas, USA, 2019, SPE193587-MS. doi: 10.2118/193587-MS

185. Z. Yang, Y. Wang, F. Zhan, W. Chen, M. Ding, C. Qian, R. Wang and B. Hou, Indolizine quaternary ammonium salt inhibitors, part III: insights into the highly effective lowtoxicity acid corrosion inhibitor - synthesis and protection performance, New J. Chem., 2019, 43, 18461. doi: 10.1039/c9nj04203b

186. A. Popova, M. Christov, S. Raicheva and E. Sokolova, Adsorption and inhibitive properties of benzimidazole derivatives in acid mild steel corrosion, Corros. Sci., 2004, 46, 1333-1350. doi: 10.1016/j.corsci.2003.09.025

187. M. Benabdellah, A. Tounsi, K.F. Khaled and B. Hammouti, Thermodynamic, chemical and electrochemical investigations of 2-mercapto benzimidazole as corrosion inhibitor for mild steel in hydrochloric acid solutions, Arab. J. Chem., 2011, 4, 17-24. doi: 10.1016/j.arabjc.2010.06.010

188. P. Rugmini Ammal, A.R. Prasad and A. Joseph, Comparative studies on the electrochemical and physicochemical behaviour of three different benzimidazole motifs as corrosion inhibitor for mild steel in hydrochloric acid, Egypt. J. Pet., 2018, 27, $1067-$ 1076. doi: $10.1016 /$ j.ejpe.2018.03.006

189. E. Ech-chihbi, A. Nahlé, R. Salim, F. Benhiba, A. Moussaif, F. El-Hajjaji, H. Oudda, A. Guenbour, M. Taleb, I. Warad and A. Zarrouk, Computational, MD simulation, SEM/EDX and experimental studies for understanding adsorption of benzimidazole derivatives as corrosion inhibitors in $1.0 \mathrm{M} \mathrm{HCl}$ solution, J. Alloys Compd., 2020, 844, 155842. doi: $10.1016 /$ j.jallcom.2020.155842

190. A. Chaouiki, M. Chafiq, M. Rbaa, R. Salghi, B. Lakhrissi, I.H. Ali, S. Bashir and I.M. Chung, Comprehensive assessment of corrosion inhibition mechanisms of novel benzimidazole compounds for mild steel in $\mathrm{HCl}$ : An experimental and theoretical investigation, J. Mol. Liq., 2018, 319, 114081. doi: 10.1016/j.molliq.2020.114383

191. I.B. Onyeachu, I.B. Obot , A.H. Alamri and C.A. Eziukwu, Effective acid corrosion inhibitors for X60 steel under turbulent flow condition based on benzimidazoles: electrochemical, theoretical, SEM, ATR-IR and XPS investigations, Eur. Phys. J. Plus, 2020, 135, 129. doi: 10.1140/epjp/s13360-020-00167-4 
192. K. Chkirate, K. Azgaou, H. Elmsellem, B. El Ibrahimi, N.K. Sebbar, A. El Hassane, M. Benmessaoud, S. El Hajjaji and E. El Mokhtar, Corrosion inhibition potential of 2[(5-methylpyrazol-3-yl)methyl]benzimidazole against carbon steel corrosion in $1 \mathrm{M} \mathrm{HCl}$ solution: Combining experimental and theoretical studies, J. Mol. Liq., 2020, 114750. doi: 10.1016/j.molliq.2020.114750

193. Y. Tang, F. Zhang, S. Hu, Z. Cao, Z. Wu and W. Jing, Novel benzimidazole derivatives as corrosion inhibitors of mild steel in the acidic media. Part I: Gravimetric, electrochemical, SEM and XPS studies, Corros. Sci., 2013, 74, 271-282. doi: 10.1016/j.corsci.2013.04.053

194. B. Xu, W. Gong, K. Zhang, W. Yang, Y. Liu, X, Yin, H. Shi and Y. Chen, Theoretical prediction and experimental study of 1-Butyl-2-(4-methylphenyl)benzimidazole as a novel corrosion inhibitor for mild steel in hydrochloric acid, J. Taiwan Inst. Chem. Eng., 2015, 51, 193-200. doi: 10.1016/j.jtice.2015.01.014

195. M. Yadav, S. Kumar, T. Purkait, L.O. Olasunkanmi, I. Bahadur and E.E. Ebenso, Electrochemical, thermodynamic and quantum chemical studies of synthesized benzimidazole derivatives as corrosion inhibitors for N80 steel in hydrochloric acid, $J$. Mol. Liq., 2016, 213, 122-138. doi: 10.1016/j.molliq.2015.11.018

196. G.A. Zhang, X.M. Hou, B.S. Hou and H.F. Liu, Benzimidazole derivatives as novel inhibitors for the corrosion of mild steel in acidic solution: Experimental and theoretical studies, J. Mol. Liq., 2019, 278, 413-427. doi: 10.1016/j.molliq.2019.01.060

197. P. Kannan, T.S. Rao and N. Rajendran, Anti-corrosion behavior of benzimidazoliumtetrafluroborate ionic liquid in acid medium using electrochemical noise technique, J. Mol. Liq., 2016, 222, 586-595. doi: 10.1016/j.molliq.2016.07.116

198. P. Kannan, J. Karthikeyan, P. Murugan, T.S. Rao and N. Rajendran, Corrosion inhibition effect of novel methyl benzimidazolium ionic liquid for carbon steel in $\mathrm{HCl}$ medium, J. Mol. Liq., 2016, 221, 368-380. doi: 10.1016/j.molliq.2016.04.130

199. X. Zheng, S. Zhang, W. Li, L. Yin, J. He and J. Wu, Investigation of 1-butyl-3-methyl$1 \mathrm{H}$-benzimidazolium iodide as inhibitor for mild steel in sulfuric acid solution, Corros. Sci., 2014, 80, 383-392. doi: 10.1016/j.corsci.2013.11.053

200. S.K. Shetty and A.N. Shetty, Eco-friendly benzimidazolium based ionic liquid as a corrosion inhibitor for aluminum alloy composite in acidic media, J. Mol. Liq., 2017, 225, 426-438 doi: 10.1016/j.molliq.2016.11.037

201. E. Ech-chihbi, A. Nahle, R. Salim, H. Oudda, F. El Hajjaji, F. El Kalai, A. El Aatiaoui and M. Taleb, An Investigation into Quantum Chemistry and Experimental Evaluation of Imidazopyridine Derivatives as Corrosion Inhibitors for C-Steel in Acidic Media, $J$. Bio Tribo Corros., 2019, 5, 24. doi: 10.1007/s40735-019-0217-9

202. Y. Xu, S. Zhang, L. Guo, B. Tan, C. Liao, Y. Zhou and L.H. Madkour, Halogensubstituted pyrazolo-pyrimidine derivatives as corrosion inhibitors for copper in sulfuric acid solution, Int. J. Corros. Scale Inhib., 2018, 7, no. 2, 236-249. doi: 10.17675/2305$\underline{6894-2018-7-2-9}$ 
203. Y. Xu, S. Zhang, W. Li, L. Guo, S. Xu, L. Feng and L.H. Madkour, Experimental and theoretical investigations of some pyrazolo-pyrimidine derivatives as corrosion inhibitors on copper in sulfuric acid solution, Appl. Surf. Sci., 2018, 459, 612-620. doi: 10.1016/j.apsusc.2018.08.037

204. G.K. Gomma, Influence of copper cation on inhibition of corrosion for steel in presence of benzotriazole in sulfuric acid, Mater. Chem. Phys., 1998, 55, no. 2, 131-138. doi: 10.1016/S0254-0584(98)00084-4

205. S.Y. Sayed, M.S. El-Deab, B.E. El-Anadouli and B.G. Ateya, Synergistic Effects of Benzotriazole and Copper Ions on the Electrochemical Impedance Spectroscopy and Corrosion Behavior of Iron in Sulfuric Acid, J. Phys. Chem. B, 2003, 107, 5575-5585. doi: $10.1021 / \mathrm{jp} 034334 \mathrm{x}$

206. Q. Qu, S. Jiang, W. Bai and L. Li, Effect of ethylenediamine tetraacetic acid disodium on the corrosion of cold rolled steel in the presence of benzotriazole in hydrochloric acid, Electrochim. Acta, 2007, 52, 6811-6820. doi: 10.1016/j.electacta.2007.04.114

207. A.K. Satpati and P.V. Ravindran, Electrochemical study of the inhibition of corrosion of stainless steel by 1,2,3-benzotriazole in acidic media, Mater. Chem. Phys., 2008, 109, 352-359. doi: 10.1016/j.matchemphys.2007.12.002

208. G. Trabanelli, A. Frignani, C. Monticelli and F. Zucchi, Alkyl-benzotriazole derivatives as inhibitors of iron and copper corrosion, Int. J. Corros. Scale Inhib., 2015, 4, no. 1, 96-107. doi: 10.17675/2305-6894-2015-4-1-096-107

209. S.T. Selvi, V. Raman and N. Rajendran, Corrosion inhibition of mild steel by benzotriazole derivatives in acidic medium, J. Appl. Electrochem., 2003, 33, 11751182. doi: 10.1023/B:JACH.0000003852.38068.3f

210. M.A. Hegazy, Novel cationic surfactant based on triazole as a corrosion inhibitor for carbon steel in phosphoric acid produced by dihydrate wet process, J. Mol. Liq., 2015, 208, 227-236. doi: 10.1016/j.molliq.2015.04.042

211. K.F. Khaled, S.A. Fadl-Allah and B. Hammouti, Some benzotriazole derivatives as corrosion inhibitors for copper in acidic medium: Experimental and quantum chemical molecular dynamics approach, Mater. Chem. Phys., 2009, 117, 148-155. doi: 10.1016/j.matchemphys.2009.05.043

212. K.F. Khaled and M.A. Amin, Dry and wet lab studies for some benzotriazole derivatives as possible corrosion inhibitors for copper in $1.0 \mathrm{M} \mathrm{HNO}_{3}$, Corros. Sci., 2009, 51, $2098-$ 2106. doi: $10.1016 /$ j.corsci.2009.05.038

213. K.F. Khaled, Experimental and atomistic simulation studies of corrosion inhibition of copper by a new benzotriazole derivative in acid medium, Electrochim. Acta, 2009, 54, 4345-4352. doi: 10.1016/j.electacta.2009.03.002

214. Z.Z. Tasic, M.B. Petrovic Mihajlovic and M.M. Antonijevic, The influence of chloride ions on the anti-corrosion ability of binary inhibitor system of 5-methyl- $1 \mathrm{H}$ benzotriazole and potassium sorbate in sulfuric acid solution, J. Mol. Liq., 2016, 222, 17. doi: 10.1016/j.molliq.2016.07.016 
215. Z.Z. Tasic, M.M. Antonijevic, M.B. Petrovic Mihajlovic and M.B. Radovanovic, The influence of synergistic effects of 5-methyl- $1 H$-benzotriazole and potassium sorbate as well as 5-methyl-1H-benzotriazole and gelatin on the copper corrosion in sulphuric acid solution, J. Mol. Liq., 2016, 219, 463-473. doi: 10.1016/j.molliq.2016.03.064

216. A.A. Khadom and A.S. Yaro, Mass transfer effect on corrosion inhibition process of copper-nickel alloy in hydrochloric acid by Benzotriazole, J. Saudi Chem. Soc., 2014, 18, 214-219. doi: 10.1016/j.jscs.2011.06.013

217. P. Hu, Z. Wu, J. Wang, Y. Huang, Q. Liu and S.-F. Zhou, Corrosion inhibiting performance and mechanism of protic ionic liquids as green brass inhibitors in nitric acid, Green Energy Environ., 2020, 5, 214-222. doi: 10.1016/j.gee.2019.11.003

218. A.A. Mahmmod, I.A. Kazarinov, A.A. Khadom and H.B. Mahood, Experimental and Theoretical Studies of Mild Steel Corrosion Inhibition in Phosphoric Acid Using Tetrazoles Derivatives, J. Bio Tribo Corros., 2018, 4, 58. doi: 10.1007/s40735-018$\underline{0171-y}$

219. S.A. Umoren and M.M. Solomon, Effect of halide ions on the corrosion inhibition efficiency of different organic species - A review, J. Ind. Eng. Chem., 2015, 21, 81100. doi: $10.1016 /$ j.jiec.2014.09.033

220. Ya.G. Avdeev and A.Yu. Luchkin, Effect of IFKhAN-92 inhibitor on electrode reactions and corrosion of mild steels in hydrochloric and sulfuric acid solutions, Int. J. Corros. Scale Inhib., 2013, 2, no. 1, 53-66. doi: 10.17675/2305-6894-2013-2-1-053-066

221. Ya.G. Avdeev, D.S. Kuznetsov, M.V. Tyurina, S.V. Oleynik and M.A. Chekulaev, High-temperature inhibitors of stainless steel corrosion in sulfuric acid solutions, Int. J. Corros. Scale Inhib., 2017, 6, no. 1, 47-58. doi: 10.17675/2305-6894-2017-6-1-4

222. Ya.G. Avdeev, D.S. Kuznetsov and S.V. Oleinik, Inhibitor Protection of Steels in Solutions of Sulfuric Acid under High-Temperature Corrosion (up to $200^{\circ} \mathrm{C}$ ), Prot. Met. Phys. Chem. Surf., 2018, 54, no. 7, 1305-1311. doi: 10.1134/S2070205118070067

223. Ya.G. Avdeev, P.A. Belinskii, Yu.I. Kuznetsov and O.O. Zel', Zashchita stali ot sernokislotnoi korrozii ingibitorom IFKhAN-92 (Protection of steel against sulfuric acid corrosion by inhibitor IFKhAN-92), Korroz.: Mater., Zashch. (Corros.: Mater., Prot.), 2008, no. 8, 16-21 (in Russian).

224. Ya.G. Avdeev, M.V. Tyurina and Yu.I. Kuznetsov, Protection of low-carbon steel in phosphoric acid solutions by mixtures of a substituted triazole with sulfur-containing compounds, Int. J. Corros. Scale Inhib., 2014, 3, no. 4, 246-253. doi: 10.17675/23056894-2014-3-4-246-253

225. Ya. Avdeev, M. Tyurina, V. Rabinkov and A. Luchkin, Inhibitive Protection of LowCarbon Steel in Citric Acid Solutions, in MATEC Web of Conferences, RSP 2017 XXVI R-S-P Seminar 2017, Theoretical Foundation of Civil Engineering, 2017, 117, 00143. doi: $\underline{10.1051 / \text { matecconf } / 201711700143}$ 
226. Ya.G. Avdeev, Yu.I. Kuznetsov and M.V. Tyurina, Ob ingibirovanii korrozii nizkouglerodistoi stali v goryachikh rastvorakh organicheskikh kislot (On the Inhibition of Mild Steel Corrosion in Hot Organic Acid Solutions), Korroz.: Mater., Zashch. (Corros.: Mater., Prot.), 2012, no. 3, 24-28 (in Russian).

227. Ya.G. Avdeev, Protection of metals in phosphoric acid solutions by corrosion inhibitors. A review, Int. J. Corros. Scale Inhib., 2019, 8, no. 4, 760-798. doi: 10.17675/23056894-2019-8-4-1

228. Ya.G. Avdeev, M.V. Tyurina and Yu.I. Kuznetsov, Zashchita nizkouglerodistoi stali v rastvore fosfornoi kisloty ingibitorom IFKhAN-92. Ch. 3. Vliyaniye kationov zheleza (Protection of low carbon steel in a solution of phosphoric acid by IFKhAN-92 inhibitor. Part 3. The effect of iron cations), Korroz.: mater., zashch. (Corros.: Mater., Prot.), 2014, no. 1, 18-25 (in Russian).

229. Ya.G. Avdeev, O.A. Kireeva, Yu.I. Kuznetsov and I.G. Gorichev, Effect of the anionic composition of acidic solutions containing Fe(III) on the protection of low-carbon steel by a triazole inhibitor, Int. J. Corros. Scale Inhib., 2016, 5, no. 4, 333-346. doi: 10.17675/2305-6894-2016-5-4-4

230. Ya.G. Avdeev, O.A. Kireeva, D.S. Kuznetsov and Yu.I. Kuznetsov, The Influence of Hexamethylenetetramine on the Corrosion Inhibition of Low Carbon Steel in Mixtures of Sulfurous and Phosphorus Acids Containing Fe(III) by IFKhAN-92 and KNCS Composition, Prot. Met. Phys. Chem. Surf., 2018, 54, no. 7, 1298-1304. doi: 10.1134/S2070205118070055

231. Ya.G. Avdeev, O.A. Kireeva, D.S. Kuznetsov and Yu.I. Kuznetsov, Usilenie urotropinom ingibirovaniya korrozii stali 08PS kompozitsiei IFKhAN-92 s KNCS v smesyakh NS1 i $\mathrm{H}_{3} \mathrm{PO}_{4}$, soderzhashchikh Fe(III) (Improvement of corrosion inhibition of steel 08PS by composition of IFKhAN-92 with KNCS in mixtures of $\mathrm{HCl}$ and $\mathrm{H}_{3} \mathrm{PO}_{4}$ containing Fe(III) by urotropine), Korroz.: Mater., Zashch. (Corros.: Mater., Prot.), 2018, no. 7, 22-28 (in Russian). doi: 10.31044/1813-7016-2018-0-7-22-28

232. Ya.G. Avdeev, L.V. Frolova, A.V. Panova, K.L. Anfilov and Yu.I. Kuznetsov, Corrosion and hydrogenation of steels in solutions of mineral acids containing iron(III) salts, Int. J. Corros. Scale Inhib., 2020, 9, no. 1, 320-333. doi: 10.17675/2305-68942020-9-1-21

233. Ya.G. Avdeev, L.V. Frolova, A.V. Panova, K.L. Anfilov and Yu.I. Kuznetsov, Inhibitory protection of steels in hydrochloric and phosphoric acid mixtures containing Fe(III) salts, Int. J. Corros. Scale Inhib., 2020, 9, no. 3, 1084-1096. doi: 10.17675/23056894-2020-9-3-19

234. G. Aromi, L.A. Barrios, O. Roubeau and P. Gamez, Triazoles and tetrazoles: Prime ligands to generate remarkable coordination materials, Coord. Chem. Rev., 2011, 255, 485-546. doi: 10.1016/j.ccr.2010.10.038

235. J.G. Haasnoot, Mononuclear, oligonuclear and polynuclear metal coordination compounds with 1,2,4-triazole derivatives as ligands, Coord. Chem. Rev., 2000, 200202, 131-185. doi: $\underline{10.1016 / \mathrm{S} 0010-8545(00) 00266-6}$ 
236. M. Rubio, R. Hernández, A. Nogales, A. Roig and D. López, Structure of a spincrossover $\mathrm{Fe}(\mathrm{II})-1,2,4$-triazole polymer complex dispersed in an isotactic polystyrene matrix, Eur. Polym. J., 2011, 47, no. 1, 52-60. doi: 10.1016/j.eurpolymj.2010.10.029

237. L.G. Lavrenova， E.V. Kirillova， V.N. Ikorskii， Yu.G. Shvedenkov, V.A. Varnek, L.A. Sheludyakova and S.V. Larionov, Iron(II) Complexes with 4-R-1,2,4-Triazoles $(R$ = Ethyl, Propyl) Exhibiting ${ }^{1} \mathrm{~A}_{1} \rightleftarrows{ }^{5} \mathrm{~T}_{2}$ Spin Transition, Russ. J. Coord. Chem., 2001, 27, no. 1, 46-50. doi: 10.1023/A:1009540925335

238. M.B. Bushuev, L.G. Lavrenova, V.N. Ikorskii， Yu.G. Shvedenkov, V.A. Varnek, L.A. Sheludyakova and S.V. Larionov, Iron(II) Complexes with 4-R-1,2,4-Triazoles $(R$ = Ethyl, Propyl, Isopropyl): Synthesis and Properties, Russ. J. Coord. Chem., 2004, 30, 284-290. doi: 10.1023/B:RUCO.0000022805.47477.75

239. T. Huxel, S. Riedel, J. Lach and J. Klingele, Iron(II) and Nickel(II) Complexes of NAlkylimidazoles and 1-Methyl-1H-1,2,4-Triazole: X-ray Studies, Magnetic Characterization, and DFT Calculations, Z. Anorg. Allg. Chem., 2012, 638, no. 6, 925934. doi: $10.1002 /$ zaac. 201200117

240. S.P. Shpan'ko, V.P. Grigor'ev, E.V. Plekhanova and V.A. Anisimova, Inhibitive and residual protective effects of benzimidazole derivative upon acid corrosion of iron, Prot. Met. Phys. Chem. Surf., 2010, 46, no. 2, 255-260. doi: 10.1134/S2070205110020139

241. Ya.G. Avdeev, A.Yu. Luchkin and Yu.I. Kuznetsov, Adsorption of IFKhAN-92 Corrosion Inhibitor on Low Carbon Steel from Hydrochloric Acid Solution, Prot. Met. Phys. Chem. Surf., 2013, 49, no. 7, 865-868. doi: 10.1134/S2070205113070046

242. Ya.G. Avdeev, A.Yu. Luchkin and Yu.I. Kuznetsov, Adsorption of IFKhAN-92 Inhibitor on Low Carbon Steel from Sulfuric Acid Solution, Prot. Met. Phys. Chem. Surf., 2020, 56, no. 7, 1244-1248. doi: 10.1134/S2070205120070035

243. Ya.G. Avdeev, A.Yu. Luchkin, M.V. Tyurina and Yu.I. Kuznetsov, Adsorption of IFKhAN-92 Corrosion Inhibitor From Acidic Phosphate Solution on Low Carbon Steel, Prot. Met. Phys. Chem. Surf., 2017, 53, no. 7, 1247-1251. doi: 10.1134/S2070205117070048

244. Ya.G. Avdeev, D.S. Kuznetsov and Yu.I. Kuznetsov, Adsorbtsiya ingibitora korrozii IFKhAN-92 na khromonikelevoi stali iz rastvorov mineral'nykh kislot (Adsorption of corrosion inhibitor IFKhAN-92 on chromium-nickel steel from mineral acid solutions), Korroz.: Mater., Zashch. (Corros.: Mater., Prot.), 2020, no. 2, 27-32 (in Russian). doi: 10.31044/1813-7016-2020-0-2-27-32

245. N.P. Andreeva, D.S. Kuznetsov and Ya.G. Avdeev, Study of the Adsorption of the IFKhAN-92 Inhibitor on the Surface of Chromium-Nickel Steel from Hydrochloric and Sulfuric Acid Solutions by Ellipsometry, Prot. Met. Phys. Chem. Surf., 2019, 55, no. 7, 1280-1286. doi: $10.1134 /$ S2070205119070025

246. G. Gece, The use of quantum chemical methods in corrosion inhibitor studies, Corros. Sci., 2008, 50, 2981-2992. doi: 10.1016/j.corsci.2008.08.043 
247. M.A. Quraishi, D.S. Chauhan and V.S. Saji, Computational methods of inhibitor evaluation, in Heterocyclic Organic Corrosion Inhibitors, Eds.: M.A. Quraishi, D.S. Chauhan and V.S. Saji, Elsevier Inc., 2020, 59-86. doi: 10.1016/B978-0-12818558-2.00003-5

248. Ya.G. Avdeev, A.Yu. Luchkin, Yu.I. Kuznetsov, L.P. Kazanskii and Yu.E. Pronin, Zashchitnoe posledeistvie ingibitora IFKhAN-92 pri korrozii stali v solyano- i sernokislykh sredakh (The protective aftereffect of the inhibitor IFKhAN-92 for steel corrosion in hydrochloric and sulphuric acid solutions), Korroz.: Mater., Zashch. (Corros.: Mater., Prot.), 2012, no. 11, 20-25 (in Russian).

249. Ya.G. Avdeev, Zashchita metallov ot kislotnoi korrozii nenasyshchennymi organicheskimi soedineniyami $i$ azolami pri povyshennykh temperaturakh. Dissernatsiya doktora khimicheskih nauk (Protection of metals from high-temperature acid corrosion by unsaturated organic compounds and azoles. Dissertation, Doctor of Sciences), A.N. Frumkin Institute of Physical Chemistry and Electrochemistry, Russian Academy of Sciences, Moscow, 2013, 366 pp. (in Russian).

250. Ya.G. Avdeev, M.V. Tyurina, Yu.I. Kuznetsov, Yu.E. Pronin and L.P. Kazanskii, Zashchita nizkouglerodistoi stali v rastvorakh fosfornoi kisloty ingibitorom IFKhAN-92. Ch. 2 (Protection of low-carbon steel in solutions of phosphoric acid by inhibitor IFKhAN-92. Part 2), Korroz.: Mater., Zashch. (Corros.: Mater., Prot.), 2013, no. 6, $17-$ 23 (in Russian).

251. Ya.G. Avdeev, Yu.B. Makarichev, D.S. Kuznetsov and L.P. Kazanskii, Osobennosti zashchity khromonikelevoi stali $\mathrm{v}$ rastvorakh solyanoi kisloty proizvodnym triazola (Specificity of protection of chromium-nickel steel in hydrochloric acid solutions by triazole derivative), Korroz.: Mater., Zashch. (Corros.: Mater., Prot.), 2018, no. 9, $22-$ 29 (in Russian). doi: 10.31044/1813-7016-2018-0-9-22-29

252. Ya.G. Avdeev, D.S. Kuznetsov, Yu.B. Makarychev and L.P. Kazansky, Protective Aftereffect of IFKhAN-92 Inhibitor for Corrosion of Nickel-Chromium Steel in Hydrochloric Acid, Prot. Met. Phys. Chem. Surf., 2020, 56, no. 7, 1264-1269. doi: 10.1134/S2070205120070047

253. Ya.G. Avdeev, Yu.B. Makarichev, D.S. Kuznetsov and L.P. Kazanskii, Osobennosti zashchity khromonikelevoi stali $\mathrm{v}$ rastvorakh sernoi kisloty proizvodnym triazola (Protective aftereffect of IFKhAN-92 inhibitor for chromium-nickel stainless steel corrosion in sulfuric acid), Korroz.: Mater., Zashch. (Corros.: Mater., Prot.), 2019, no. 1, 15-21 (in Russian). doi: 10.31044/1813-7016-2019-0-1-15-21

254. Ya.G. Avdeev, Yu.B. Makarichev and D.S. Kuznetsov, Zashchitnoe posledeistvie ingibitora IFKhAN-92 pri korrozii khromonikelevoi stali v sernoi kislote (Protective aftereffect of IFKHAN-92 inhibitor for corrosion of chromium-nickel steel in sulfuric acid), Korroz.: Mater., Zashch. (Corros.: Mater., Prot.), 2020, no. 12, 23-31 (in Russian). doi: 10.31044/1813-7016-2020-0-12-23-31 
255. Ya.G. Avdeev and M.V. Tyurina, Vliyaniye ingibitora IFKhAN-92 na elektrodnyye reaktsii i korroziyu nizkouglerodistoi stali $\mathrm{v}$ rastvore fosfornoi kisloty (Effect of IFKhAN-92 inhibitor on electrode reactions and corrosion of low-carbon steel in phosphoric acid solution), Korroz.: mater., zashch. (Corros.: Mater., Prot.), 2015, no. 6, 19-25 (in Russian).

256. Ya.G. Avdeev, T.E. Andreeva and Yu.I. Kuznetsov, A potentiometric study of an $\mathrm{H}_{2} \mathrm{SO}_{4}-\mathrm{H}_{3} \mathrm{PO}_{4}-\mathrm{H}_{2} \mathrm{O}$ system containing $\mathrm{Fe}(\mathrm{III})$ and $\mathrm{Fe}(\mathrm{II})$ cations, Int. J. Corros. Scale Inhib., 2018, 7, no. 3, 366-375. doi: 10.17675/2305-6894-2018-7-3-7

257. Ya.G. Avdeev, T.E. Andreeva, A.V. Panova and Yu.I. Kuznetsov Effect of anionic composition of solutions of mineral acids containing $\mathrm{Fe}(\mathrm{III})$ on their oxidizing properties, Int. J. Corros. Scale Inhib., 2019, 8, no. 1, 139-149. doi: 10.17675/23056894-2019-8-1-12

258. Ya.G. Avdeev, T.E. Andreeva, A.V. Panova and E.N. Yurasova, A study of the $\mathrm{H}_{2} \mathrm{SO}_{4-}$ $\mathrm{H}_{3} \mathrm{PO}_{4}-\mathrm{H}_{2} \mathrm{O}-\mathrm{Fe}(\mathrm{III})$ system by cyclic voltammetry, Int. J. Corros. Scale Inhib., 2019, 8, no. 2, 411-421. doi: 10.17675/2305-6894-2019-8-2-18

259. Ya.G. Avdeev, T.E. Andreeva, A.V. Panova and Yu.I. Kuznetsov, Cyclic voltammetric study of the $\mathrm{HCl}-\mathrm{H}_{3} \mathrm{PO}_{4}-\mathrm{H}_{2} \mathrm{O}-\mathrm{Fe}(\mathrm{III})$ system, Int. J. Corros. Scale Inhib., 2020, 9, no. 2, 538-549. doi: 10.17675/2305-6894-2020-9-2-9

260. Ya.G. Avdeev, T.E. Andreeva and A.V. Panova, Effect of urotropine on the potential of the $\mathrm{Fe}(\mathrm{III}) / \mathrm{Fe}$ (II) redox couple in mineral acid solutions, Int. J. Corros. Scale Inhib., 2020, 9, no. 2, 571-583. doi: 10.17675/2305-6894-2020-9-2-12 